\title{
La Porte d'Orée : transformations et avatars d'un secteur portuaire à Fréjus (Var)
}

\author{
par Isabelle BÉRAUD*, Chérine GÉBARA* et Corinne LANDURÉ **
}

(11) Une fouille d'urgence programmée, menée en 1986 à la Porte d'Orée à Fréjus, a permis la découverte d'une si... cession de sites, notamment une partie du bassin portuaire de Forum Julii, puis un monument public, ainsi Ge des réoccupations multiples jusqu'au $\mathrm{XIX}^{\mathrm{e}} \mathrm{s}$. Une esplanade et une darse, mises en place dans les années 30 c. notre ère, ont été remblayées pour permettre la construction d'un ensemble monumental. Il s'agit d'un aitiment à plan axial fondé partiellement sur les murs de l'état précédent, qui évoquerait un bâtiment public à ca actère somptuaire rappelant certains nymphées de tradition hellénistique. Cet ensemble daterait des années 70 de notre ère. Ce nymphée est réutilisé pendant plusieurs siècles et servira, surtout durant l'Antiquité tardive, de lieu d'habitation, de sépulture et de dépotoir. Une série de silos est installée par-dessus l'esplanade remblayée aux $\mathrm{X}^{\mathrm{e}}-\mathrm{XI}^{\mathrm{e}} \mathrm{s}$.; le site est ensuite transformé en jardins, puis récemment, en parc de stationnement.

In 1986 a rescue dig was conducted at "La Porte d'Orée" (Fréjus), which resulted in the discovery of three sites superimposed : a dock belonging to the Roman harbour of Forum Julii; a large monument, and lastly the continuing occupation of the site for private purposes. The dock, linked to the city by an esplanade, (circa 30 A.D.) was filled up around the year 70 A.D. This allowed the construction of a public building closely resembling the nymphaeum of hellenistic tradition. This deserted nymphaeum was subsequently occupied by squatters, witness the traces left of their living quarters, burials and rubbish heaps dating form the 3rd c. to the 6th c. A.D. During the 10th or 11th c. the esplanade having been banked up, a series of silos were hollowed out. The area was finally transformed into gardens and more recently, into a parking lot.

Mots clés : Port, nymphée, silos, rotonde, esplanade, darse, môle, péristyle, jardin, gallo-romain, ${ }^{\text {er }}$ s., Antiquité tardive, Haut Moyen Age, Fréjus, Var.

* Service archéologique municipal, Ville de Fréjus.

** Archéologue contractuelle. 
En 1986, un projet municipal concernant l'aménagement de la basse ville a été mis en cuvre au quartier de la Porte d'Orée à Fréjus, dans le périmètre classé : il s'agissait de la construction d'un parc de stationnement semi-enterré (fig. 1).

L'emprise $\left(2000 \mathrm{~m}^{2}\right)$ et la profondeur (atteignant jusqu'à $5 \mathrm{~m}$ ) initialement prévues pour ce parc ont nécessité une campagne de sondages puis de fouilles, menée par le service archéologique municipal de la Ville dans un délai de quatre mois, de mai à aoùt 1986. La prolongation de ce chantier par une troisième tranche de travaux n'ayant pas été jugée nécessaire par le ministère de tutelle, nous n'avons pu exploiter à notre satisfaction l'important gisement reconnu, qui reste donc partiellement exploré.

Le terrain fouillé est situé au sud de la ville antique dans l'alignement général du cardo maximus, en bordure de l'ancien port romain et au nord-est des thermes de la Porte d'Orée.

En raison des délais invoqués plus haut et de l'omniprésence de la nappe phréatique $(1,70 \mathrm{~m} \mathrm{NGF}$ à son plus bas niveau en 1986), nous avons été contraintes d'effectuer des choix et de limiter volontairement l'exploration des niveaux profonds à de simples sondages (c'est pourquoi, sur toutes les coupes présentées, les zones non fouillées sont symbolisées par des hachures).

La fouille et l'étude du matériel nous ont permis cependant de mettre en évidence cinq ètats principaux, du début du $\mathrm{I}^{\mathrm{er}} \mathrm{s}$. après $J .-C$. jusqu'au $\times \mathrm{I}^{\mathrm{C}}$ XII ${ }^{\mathrm{r}}$ s. (fig. 2 et 3). Les deux premiers états sont marqués par une phase de construction matérialisée par la mise en place de structures monumentales (bassin lié au port, monument public); les trois derniers sont des états de réoccupation, de destruction, puis de modification du faciès du site. Pour chacun des quatre premiers états, nous présentons le matériel céramique recueilli dans les différents contextes, à travers des tableaux qui prennent en compte le nombre global des tessons pour chaque catégorie considérée. Cependant pour l'état IV, cette étude de la céramique a été plus approfondie : en effet pour

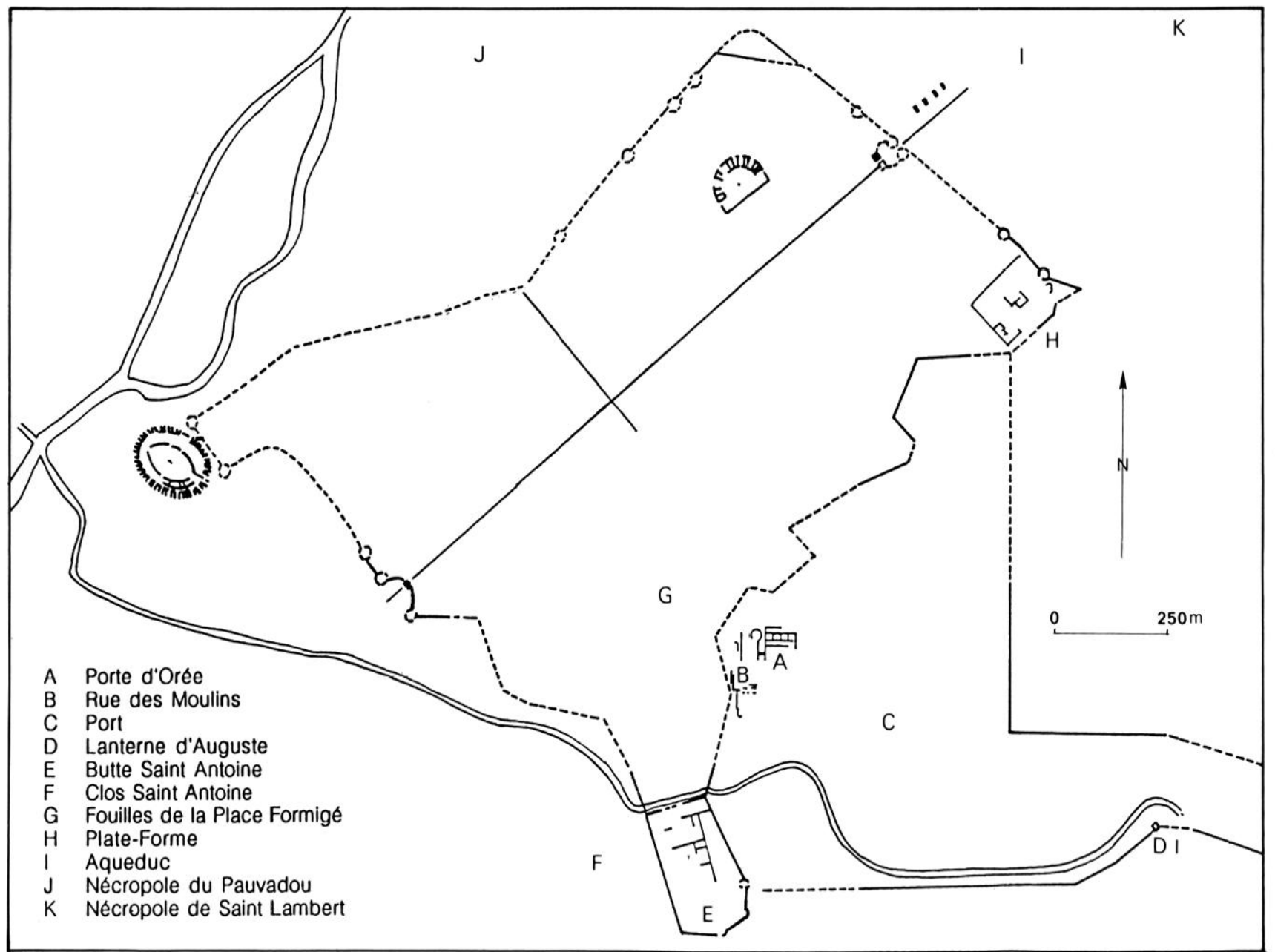

Fig. 1 - Plan général de la ville antique. 


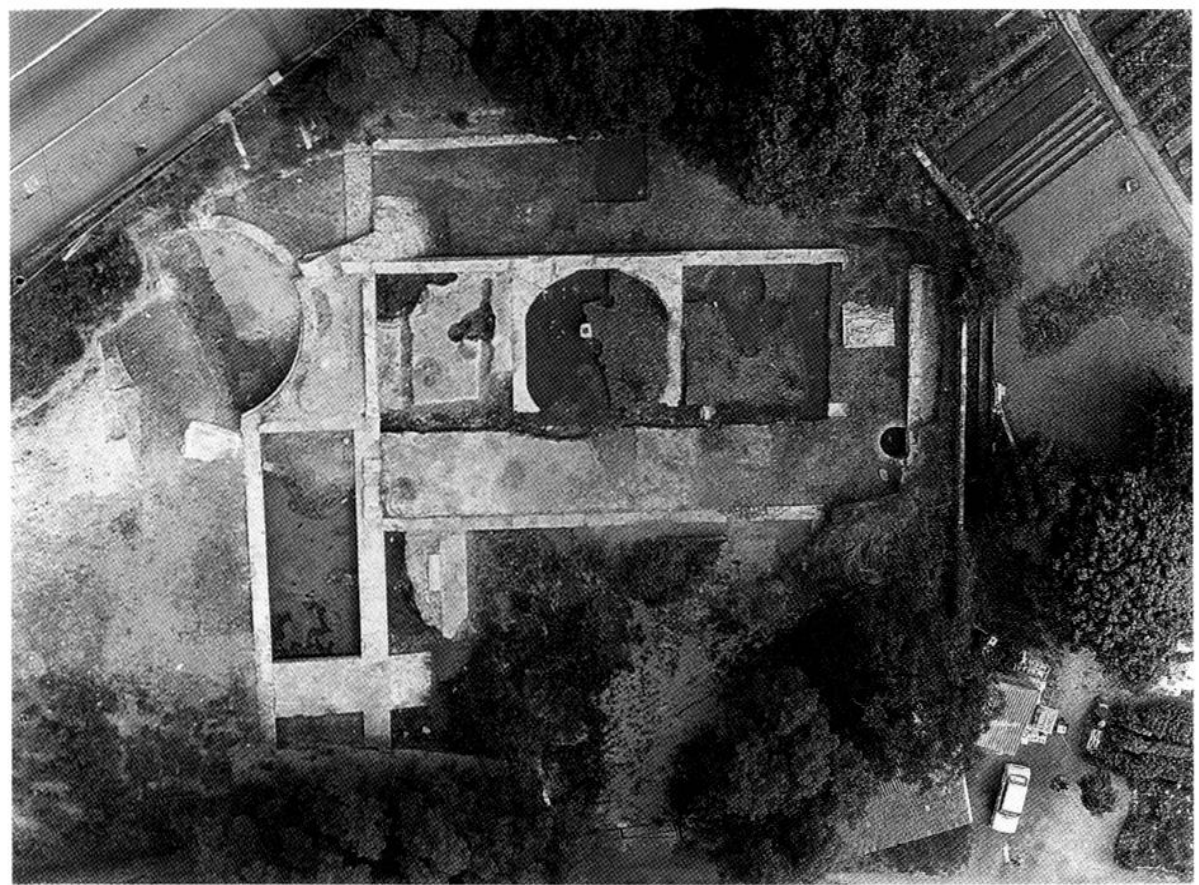

Fig. 2 Vue aerienne du
site.

liig. 3-Plan général du site de la Porte d'Orée. fouilles de $19 \times 6$.

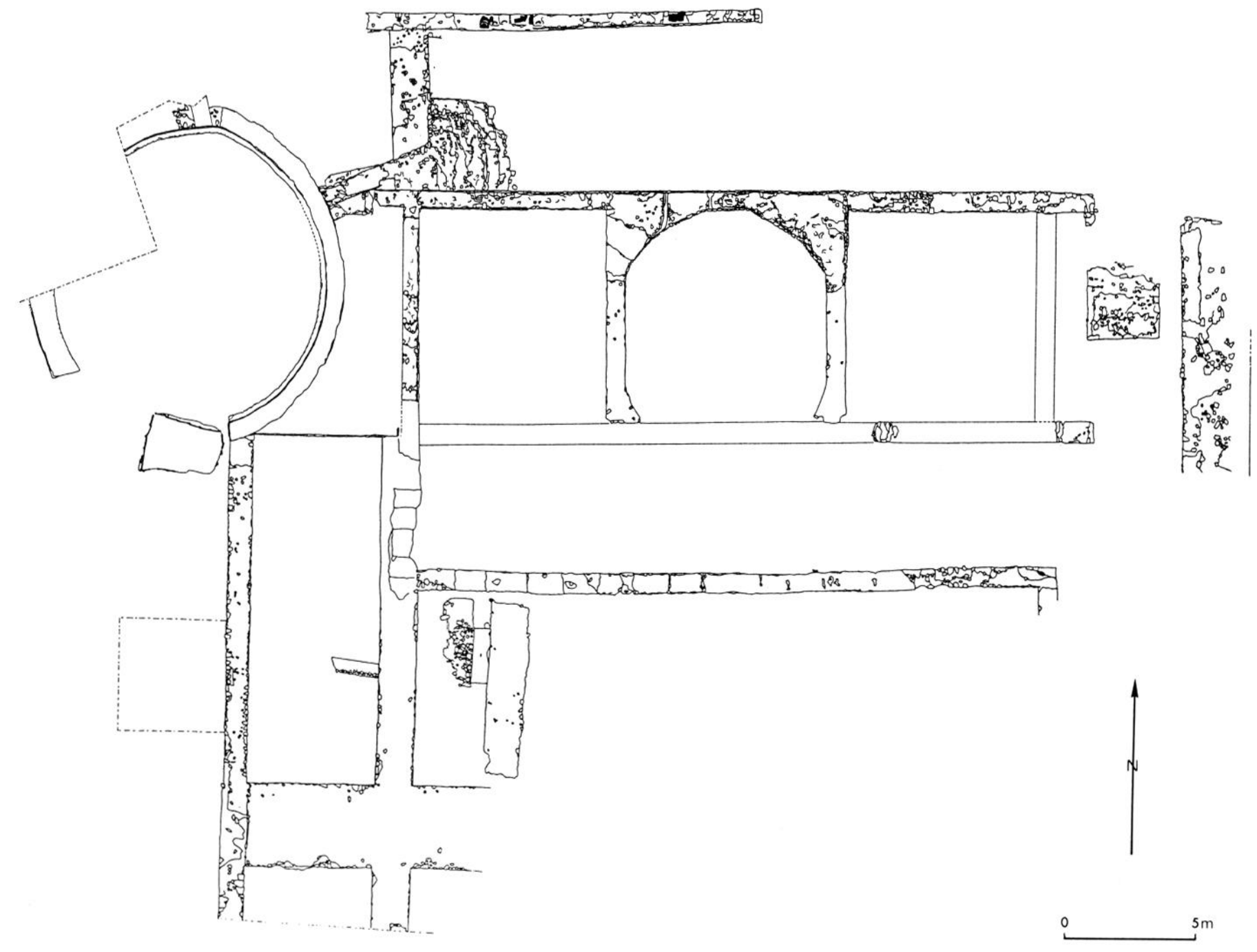




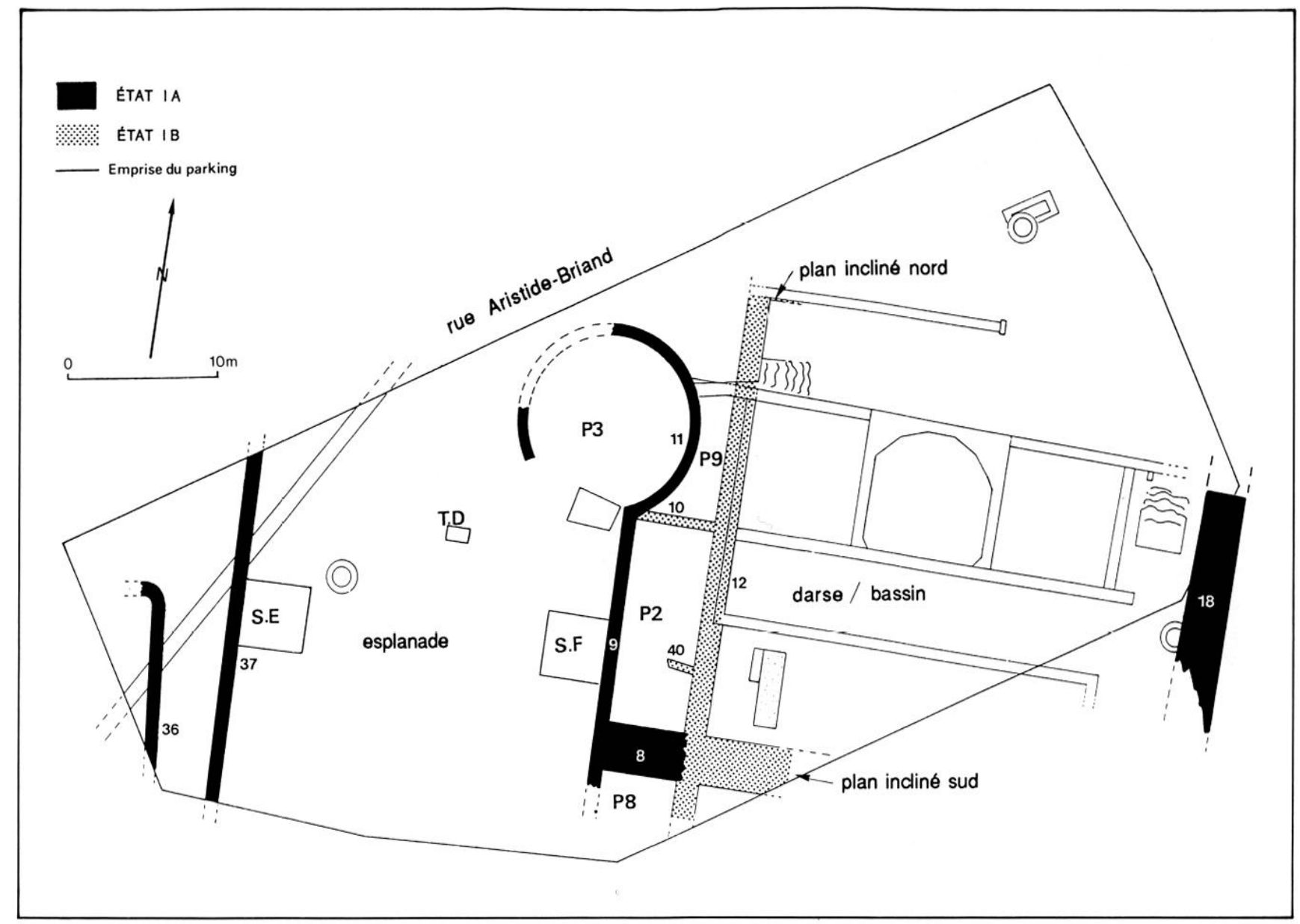

Fig. 4 - Plan sélectif des ètats IA et IB.

ces niveaux, un matériel nombreux et significatif compense l'absence de structures nouvelles qui dénote une activité moins intense et de caractère plus fruste.

\section{L'ÉTAT I}

Avant de commencer la description de l'état I (fig. 4), précisons que nous n'avons affaire qu'à une faible partie (constituée de structures incomplètes) d'un secteur se rattachant probablement au vaste ensemble du port antique de Fréjus.

Cet état est composé d'une série de murs de largeurs différentes, orientés précisément nord-sud et est-ouest. Ils sont intacts dans leur élévation et construits en opus vittalum de moellons de grès avec un mortier lissé sur leur surface supérieure. Outre ces murs, l'état I comprend aussi trois plans inclinés, une pièce circulaire à l'est de laquelle s'étendait une vaste esplanade installée sur un vide sanitaire, ainsi qu'une canalisation.
Cet ensemble, qui a subi des remblaiements successifs de sable, fut scellé lors de l'état II par une surface de circulation. Ce secteur n'était plus visible lorsque commence l'état II à l'exception de la pièce P3, une rotonde dont il subsistait par endroits une élévation de plusieurs mètres, lors des fouilles de 1986.

L'état I (pour la majorité des structures) peut se diviser en état IA et IB. Pour plus de commodité, nous examinerons les murs d'ouest, en est pour chacun des états.

\section{ÉTAT IA}

Le mur 37, dont nous ne connaissons pas les extrémités, mesure $90 \mathrm{~cm}$ de large et est orienté nord-sud. Un sondage directeur (S.E) a été ouvert contre son parement est. Parallèle au précédent, le mur 9, large de $90 \mathrm{~cm}$, est bâti en appareil aux joints beurrés (fig. 5), et possède un ressaut à $1,04 \mathrm{~m} \mathrm{NGF}$, qui se prolongeait par un contrefort doté d'un léger glacis (fig. 6). 


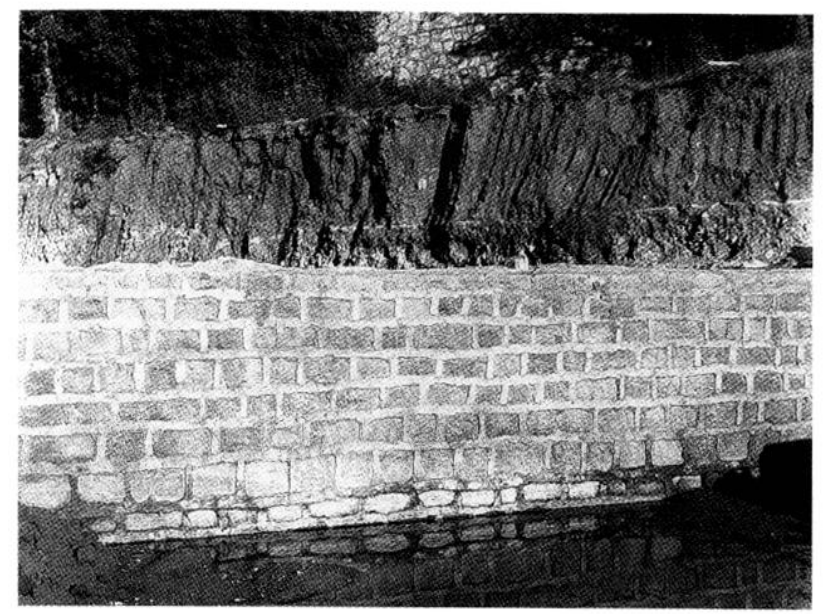

Fig. 5 - Espace P2, mur 9 : joints beurrès ou rubanés.

Un vide sanitaire d'amphores a èté révélé par un sondage (S.F) contre le mur 9. Une partie était immergée dans la nappe phréatique, ce qui a rendu leur extraction très difficile et parfois impossible ${ }^{1}$. Dans ce sondage de $16 \mathrm{~m}^{2}$, ont été mises au jour, sur $8 \mathrm{~m}^{2}$, dix-sept amphores au minimum, de divers types (parmi lesquels des Dr. 2/4, des amphores gauloises). Elles étaient disposées à peu près verticalement ou légèrement inclinées, le pied en haut et le col en bas pour la plupart.

Au nord, le mur 9 se prolonge par le mur 11 qui forme la pièce circulaire P3, d'un diamètre de $12 \mathrm{~m}$, rotonde ou tour largement ouverte vers l'esplanade et vers le sud. D'une largeur de $70 \mathrm{~cm}$ dans son élévation et de $90 \mathrm{~cm}$ au niveau du ressaut, le mur 11 est construit en moellons pratiquement carrés. Une des particularités de ce mur réside dans le fait qu'il a conservé un enduit blanc aussi bien à l'intérieur qu'à l'extérieur.

Enfin, le mur 18 à l'extrémité du site marque la limite orientale; il est orienté nord-sud et large de $2,50 \mathrm{~m}$. De cet état date un premier plan incliné. Il s'agit du mur 8 , large de $3 \mathrm{~m}$ et situé au sud du mur 9, s'appuyant perpendiculairement contre ce dernier. Les moellons ne sont pas visibles car recouverts d'enduit ou de mortier.

\section{ÉTAT IB}

Pendant l'état IB a lieu la suppression du premier plan incliné (mur 8) qui devient un large mur mis au même niveau que les autres structures. Contre ce mur vient s'appuyer le mur $12(1,45 \mathrm{~m})$,

1 En ce qui concerne l’étude des amphores composint ce vide sanitaire et pour une synthèse sur l'utilisation de ce système dans le monde romain. of. larticle de lE. Laubenheimer faisant suite à celui-ci.

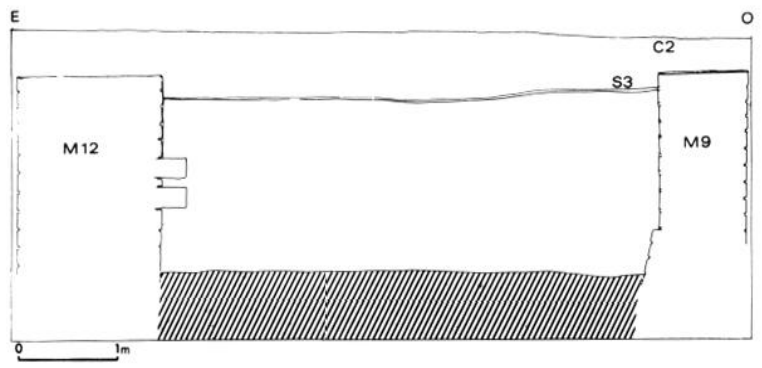

Fig. 6 - Espace P2 : coupe stratigraphique est/ouest.

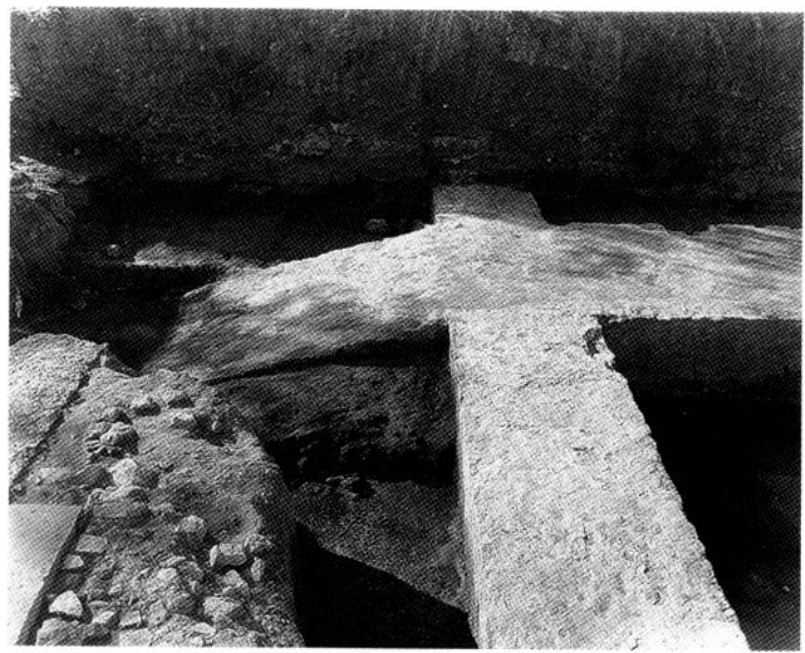

Fig. 7 - Plan inclinè de l'état IB.

orienté nord-sud, dont nous ne connaissons pas les extrémités. Il est bâti en moellons aux joints beurrés. Dans sa partie sud, sur le parement ouest, étaient disposés deux blocs en saillie placés l'un audessus de l'autre, à respectivement $1,44 \mathrm{~m}$ et $1,71 \mathrm{~m}$ $\mathrm{NGF}$, et dont la fonction nous échappe. Ce mur est également percé de trous de boulins à $1,90 \mathrm{~m} \mathrm{NGF}$, ce qui atteste sa construction en élévation et son remblaiement subséquent. Contre ce long mur, à $27 \mathrm{~m}$ de distance, ont été construits deux plans inclinés, l'un au sud dans le prolongement du premier (M8) (fig. 7) et l'autre au nord du mur 12, dont le départ est discernable sous un mur postérieur appartenant à l'état II (M1) (fig. 4). Une portion de muret (M40), perpendiculaire au mur 12, subsistait au niveau de la nappe phréatique. Ce muret, bâti en pierres sèches et orienté à peu près est-ouest, peut être interprété comme un mur de travail.

Le mur 10, large de $60 \mathrm{~cm}$, aux joints beurrés. orienté est-ouest, faisait la liaison entre la base du mur 11 et le mur 12. La structure appelée M36 se replace globalement dans l'état I : il s'agit d'une canalisation large d'environ $90 \mathrm{~cm}$, profonde de 


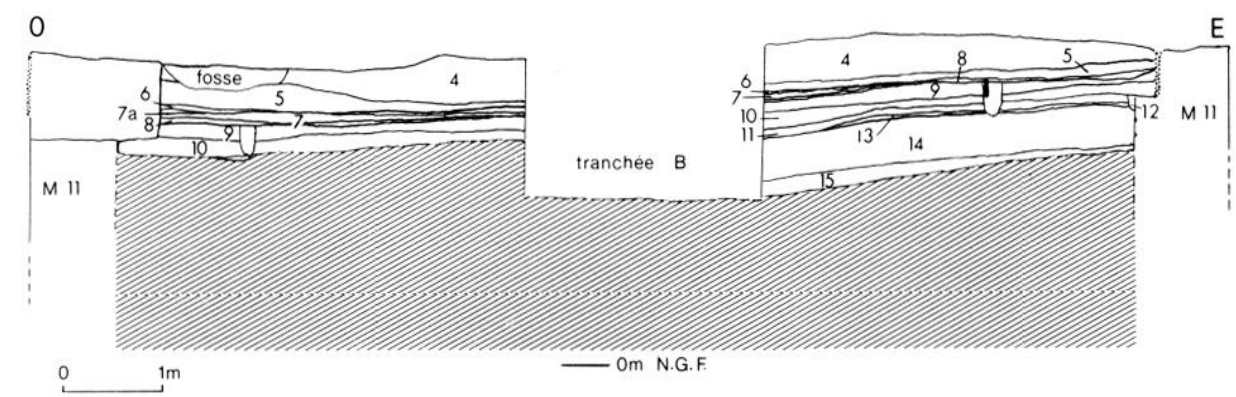

Fig. 8 - Rotonde P3 : coupe stratigraphique ouest/est.

$30 \mathrm{~cm}$, orientée nord-sud, qui bifurque brusquement vers l'ouest par un coude dans sa partie nord. Elle était recouverte de dalles de pierre de taille irrégulière d'environ $65 \mathrm{~cm}$ de large et $10 \mathrm{~cm}$ d'épaisseur. Le fond ètait recouvert de dalles similaires. Lors de sa mise au jour, cette canalisation fonctionnait toujours et avait conservé son étanchéité, transportant une eau potable qui s'écoulait vers le sud ${ }^{2}$.

\section{DATATION DE L'ÉTAT IA}

La chronologie s'appuie sur la stratigraphie de la pièce $\mathrm{P} 3$, des sondages $\mathrm{E}$ et $\mathrm{F}$ et du sondage $\mathrm{T}$.D.

\section{La pièce $\mathrm{P} 3$}

- La couche la plus ancienne rencontrée (C15) est formée de terre noire légèrement charbonneuse d'une épaisseur de 15 à $5 \mathrm{~cm}$, avec un pendage est/ ouest (fig. 8). Le matériel céramique est abondant : 2283 tessons. Il est représenté surtout par des amphores $(43 \%)$, de la céramique commune claire $(23,3 \%)$, de la céramique grise de Vaison $(9,6 \%)$ et, variant autour de $5 \%$, de la céramique modelée, de la céramique engobée locale $(4,6 \%)$ et de la sigillée sud-gauloise $(5,2 \%)$. Cette couche est datée du $\mathrm{I}^{\mathrm{er}} \mathrm{s}$. après J.-C. par une forme de céramique africaine culinaire (Hayes 19/22), une Ritt. 1 et de la céramique à paroi fine de type coquille d'ouf. On peut ajouter pour cette couche un nombre important de tessons de verre (414) ce qui représente $15,35 \%$ du matériel total. Quelques types ont été identifiés : une forme lsings 17 datée du $\mathrm{I}^{\mathrm{er}} \mathrm{s}$. après J.-C. et une assiette Isings 22 des années 0 à 70 de notre ère.

- Ia couche 14 , un épais remblai de $40 \mathrm{~cm}$ de sable fin, présente elle aussi un léger pendage. Elle comporte peu de matériel, composé essentiellement par des amphores $(38 \%$ ), de la céramique engobée locale $(21 \%)$, de la céramique à paroi fine $(12 \%)$ et de la sigillée sud-gauloise $(10 \%)$. Aucune datation précise ne se dégage de ce matériel.

- La couche 13 est une fine pellicule de terre

2 Analyses d'eau etablies par le Laboratoire departemental vétérinaire du Var, le 19 septembre 1986. marron, charbonneuse, d'une épaisseur de $5 \mathrm{~cm}$ au plus à l'est du sondage. Elle contient en majorité de la céramique claire commune $(54,4 \%)$, de la grise de Vaison $(12,4 \%$ ), de la céramique culinaire brune $(9,2 \%)$, mais aucun tesson d'amphore. Cette couche se place dans le premier quart du $\mathrm{I}^{\mathrm{er}} \mathrm{s}$., datée par deux formes d'arétine (Goudineau 32 et 41 ) et. une lampe Deneauve VA.

- La couche 12 , formée d'une bande continue large de $10 \mathrm{~cm}$ de terre et d'argile, ne contient aucun matériel.

- I a couche 11 est la dernière couche de l'état I en P3. C'est un sol d'argile jaune damé, de 7 à $8 \mathrm{~cm}$ d'épaisseur, marqué de légères dépressions linéaires. Il recouvre ces remblais successifs et présente aussi un fort pendage est/ouest, à tel point que la couche venait buter à l'ouest contre le ressaut de fondation du mur 11 . Le matériel céramique est représenté surtout par de la commune claire ( $24 \%$ ), des amphores $(22 \%)$ et de la céramique grise de Vaison (15,3\%). Cette couche est datée du milieu du $\mathrm{r}^{\mathrm{rr}} \mathrm{s}$. (40 à 70) par une forme de sigillée arétine type Goudineau 22-23, une paroi fine forme Mayet XX et une lampe type Deneauve VB.

On constate que les différentes couches de remblai se sont superposées rapidement durant la première moitié du I $^{\text {er }}$ s. après J .-C., avec une utilisation du sol de circulation à la mème époque (milieu du 1er s.).

\section{Le sondage $\mathrm{E}$}

Il a été ouvert contre la face est du mur 37 dans les couches de remblaiement (couches 10 à 16) constituant l'esplanade, sur une superficie de $16 \mathrm{~m}^{2}$ (fig. 9).

- La couche 16 , la plus ancienne et la plus riche, faite d'une terre noire et charbonneuse, n'a été entamée qu'en partie et remontait contre le mur 37. La moitié du matériel est représentée par des amphores $(53,3 \%$ \% ; note aussi en quantites significatives de la céramique grise de Vaison (18\%), de la ceramique commune à pâte claire $(10,5 \%$ ) et de la sigillée sudgauloise $(5,7 \%)$. La couche est datée a partir de 


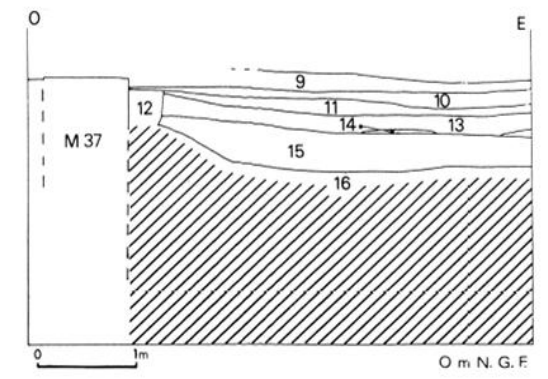

Fig. 9 - Sondage E : coupe stratigraphique ouest/est.

40 après J.-C. par un pied de Drag. 18/31, mais la majorité du matériel est datée de l'époque de Claude (Drag. 24/25, Drag. 11).

- La couche 15 , recouvrant la couche 16 et suivant son pendage à l'ouest, est formée d'une épaisseur de $40 \mathrm{~cm}$ de sable et comprenait une majorité d'amphores $(38,6 \%)$ et de céramique commune claire $(52,5 \%)$, ainsi que $11,8 \%$ de céramique grise de Vaison. Un élément de datation de la première moitié du $\mathrm{I}^{\mathrm{er}} \mathrm{s}$. de notre ère est apporté par la présence d'une sigillée de type Drag. 18.

- La couche 14 , formée de lambeaux d'une fine couche noire et charbonneuse d'une épaisseur de $2 \mathrm{~cm}$, comportait peu de matériel : $38 \%$ d'amphores, $28,5 \%$ de céramique commune claire et $14,7 \%$ de céramique grise de Vaison. La céramique à paroi fine de type coquille d'œuf permet de dater cette couche de l'époque Claude-Néron.

- La couche 13, constituée de nouveau d'un bunc de sable de $20 \mathrm{~cm}$, ne contient que très peu de matériel, essentiellement des tessons de céramique commune claire $(50 \%)$ et des amphores $(20 \%)$.

- La couche 12 est formée par une poche de sable et de terre contre le mur 37 contre laquelle viennent buter les couches 11, 13, 14 et 15. Deux types de céramique y dominent: les amphores $(47,6 \%)$ et la céramique commune claire $(40,6 \%)$. Deux bords de sigillée de type Drag. 24/25 apportent un élément de datation de la première moitié du $\mathrm{r}^{\mathrm{er}} \mathrm{s}$. de notre ère.

- La couche 11, une bande d'argile assez fine et irrégulière dont l'épaisseur varie entre 20 et $10 \mathrm{~cm}$, est dotée elle aussi de peu de matériel; la céramique commune claire y domine $(52,4 \%)$.

- La couche 10 passe par-dessus la poche C12 et vient s'appuyer contre le mur 37 . D'une faible épaisseur de sable de $15 \mathrm{~cm}$ maximum, elle ne contenait que peu de matériel, représenté en grande partie comme dans les couches précédentes, par de la céramique commune claire $(53 \%)$ et par des amphores $(29,4 \%)$. Cette couche est la dernière du sondage et ne livre, comme la couche précédente, aucun élément

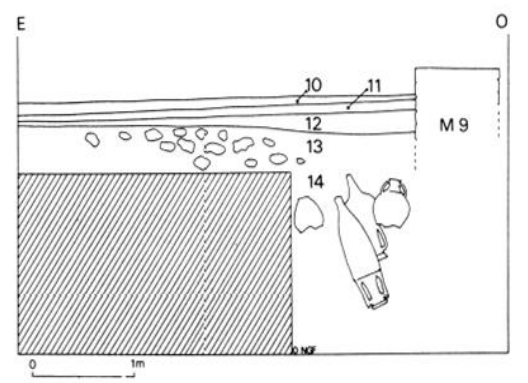

Fig. 10 - Sondage F : coupe stratigraphique ouest/est.

de datation précis. Elle est scellée par la couche 9 appartenant au sol de l'esplanade durant l'état II.

\section{Le sondage $F$}

Un deuxième sondage de dimensions similaires effectué à l'est de l'esplanade (fig. 10), contre le mur 9 , a permis, ainsi que nous l'avons déjà indiqué, de mettre au jour une partie d'un vide sanitaire d'amphores disposé dans du sable et composant la couche la plus ancienne fouillée (couche 14) (fig. 11). Une précieuse indication de datation et un terminus a quo pour l'ensemble du site sont donnés par une amphore à fond plat, de fabrication locale, portant une inscription peinte avec une date consulaire de 28 après J.-C. ${ }^{3}$.

- Sur ce lit d'amphores, une couche de terre marron (couche 13) a été déposée. Elle ne contenait, outre une grande quantité de scories de terre cuite et des nodules d'argile rubéfiée, que très peu de céramique dont l'essentiel était constitué de tessons d'amphores $(78 \%)$.

- Une nouvelle couche de sable (C12) recouvre la précédente; elle possède un léger pendage vers l'est, où elle atteint une épaisseur d'environ $25 \mathrm{~cm}$. Le matériel y est pratiquement inexistant: quelques fragments d'amphore seulement.

- La couche 11 , d'une épaisseur moyenne de $10 \mathrm{~cm}$, recélait dans de la terre noire, une importante quantité de matériel, représenté en majorité par des amphores $(69,5 \%$ : Bétique, Dr. 2/4, Tarraconaise et des fragments d'une amphore de type Richborough 527 , peu connue jusqu'à présent sur nos côtes, toutes datées du $\mathrm{I}^{\mathrm{er}} \mathrm{s}$. de notre ère). La proportion de céramique commune claire est de $30,4 \%$; la céramique culinaire brune, de $20,1 \%$; la céramique grise de Vaison, de $20 \%$; et la céramique à paroi fine, de $10,5 \%$. Un ensemble homogène de formes de sigil-

3 Pour la lecture et l'étude de l'inscription peinte sur l'amphore $n^{\circ} 9$ du sondage F. cf. l'annexe III de l'article de F. Laubenheimer (infra, p. 261), par B. Liou, que nous remercions ici. 


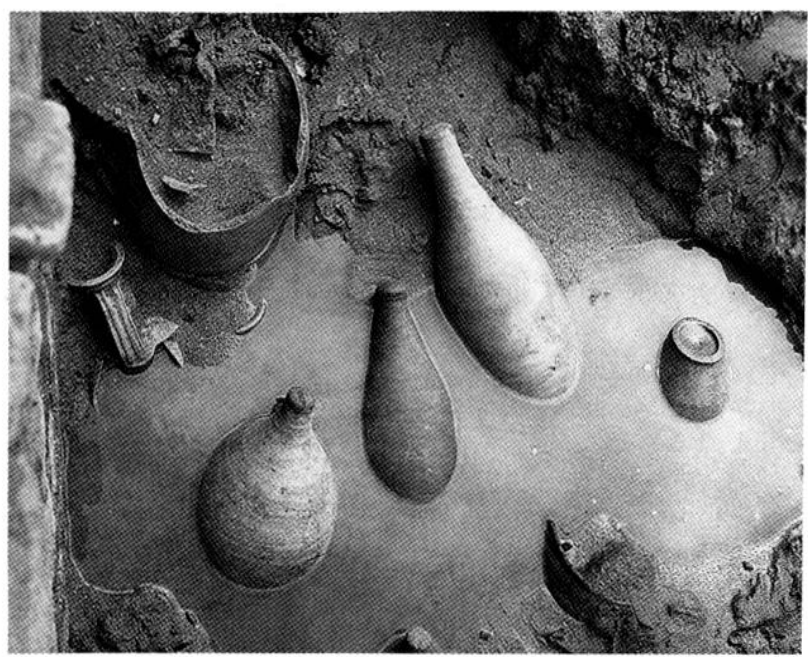

Fig. 11 - Sondage F : vide sanitaire d'amphores.

lées (Drag. 24/25 et 18/31, Hermet 2/12) et de paroi fine (Mayet XXXVII et coquille d'œuf) ainsi que des lampes (Deneauve IVA, VE, et VG) permet de définir une datation moyenne autour du milieu du $\mathrm{I}^{\mathrm{er}} \mathrm{s}$. après J.-C.

- La couche 10 clôture cet état; elle est faite d'un lit de sable de $10 \mathrm{~cm}$, qui servait, on peut le supposer, de sol de circulation avec peu de matériel, principalement des amphores $(42 \%)$, de la céramique commune claire $(32,2 \%)$ et de la céramique à paroi tine $(9,6 \%)$.

Un indice supplémentaire de datation démontrant l'étendue du vide sanitaire sous l'esplanade est apporté par une amphore mise au jour par un engin mécanique lors d'un sondage (T.D) en son centre. Elle se situait à une profondeur équivalente à celle trouvée dans le sondage $F$ et était déposée de la même manière sous un feuilleté de couches de remblai. Il s'agit d'une amphore de type Dr. 7 datée du $\mathrm{I}^{\mathrm{er}} \mathrm{s}$. (Peacock, Williams, 1986, p.117) et plus précisément, dans le contexte de l'épave de la Chrétienne $\mathrm{H}$ à Agay (Santamaria, 1985, p. 260 et fig. 5), du premier quart du $\mathrm{I}^{\mathrm{er}} \mathrm{s}$. de notre ère.

\section{DATATION DE L'ÉTAT IB}

Le seul élément de datation est fourni par l'espace P2, remblayé par du sable presque stérile. Hormis quelques tessons d'amphores et un fond de cruche en céramique engobée locale, un bord de mortier en sigillée sud-gauloise de la forme Ritt.12 permet de replacer cet état au milieu du $\mathrm{I}^{\mathrm{er}}$ s. après $\mathbf{J}$.-C. (40-70).

Le module de l'appareil des murs est un élément supplémentaire de datation. L'ensemble des murs de la Porte d'Orée est bâti en opus villatum. Cet appareil se répand en Gaule (Adam, 1984, p.147 et 149) au
$\mathrm{I}^{\mathrm{er}} \mathrm{s}$. de notre ère et en particulier à Fréjus où l'on peut observer l'usage systématique qui en est fait sur les édifices antiques. Une étude de P.-A. Février (Février, 1956b) portant sur les appareils romains de Fréjus a permis d'établir une chronologie approximative selon le module des moellons. Si l'on met en parallèle ces modules et ceux de la Porte d'Orée ${ }^{4}$, on constate une similitude avec le petit appareil régulier à moellons épais évoqué par P.-A. Février qui daterait à partir de la fin du $\mathrm{I}^{\text {er }} \mathrm{s}$. avant J.-C. ou du début du $\mathrm{I}^{\mathrm{er}} \mathrm{s}$. après. La base de la Lanterne d'Auguste et les quais du port seraient aussi de cette époque au vu de l'appareil utilisé.

\section{CONCLUSION}

Si l'on s'appuie sur les données fournies par la céramique pour l'état IA, il apparaît que l'occupation du site commence lors de la mise en place du vide sanitaire, à partir du deuxième quart du $\mathrm{I}^{\mathrm{er}} \mathrm{s}$. après $\mathbf{J}$.-C. (dans les années 30 ). Ensuite, les couches de remblai de l'esplanade indiquent que les travaux ont dù s'accomplir très rapidement, aux alentours des années 50 de notre ère. Les aménagements de l'état IB interviennent aussitôt après, à cause peutètre d'une faiblesse des structures, en particulier du mur 9, ou d'un envasement qui aurait entraîné un réaménagement du bassin. L'état II se traduira par une transformation radicale du site.

Si l'on suit ce raisonnement et les données stratigraphiques, il faut admettre que cet ensemble n'a $\mathrm{pu}$ fonctionner qu'une cinquantaine d'années au maximum, durant lesquelles il a subi une importante restructuration. Cette brève existence explique sans doute le très bon état des structures qui n'ont que peu subi l'action de l'eau et l'usure du temps avant d'être définitivement remblayées.

\section{LA CÉRAMIQUE COMMUNE DE L'ÉTAT I}

Fig. 12 et tabl. I, p. 216.

Il ressort des pourcentages de matériel présenté ci-dessus une prédominance de la céramique utilitaire, représentée par des formes de commune claire tournée, et des amphores locales ou importées. Les tessons présentés proviennent tous (hormis deux exemplaires) de la couche 11 du sondage $F$ et de la couche 15 de la pièce 3 .

4 Détail des dimensions des appareils (hauteur $\times$ largeur) : mur $8: 13 / 20 \mathrm{~cm} \times 15 / 30 \mathrm{~cm} ;$ mur $9: 12 \mathrm{~cm} \times 16 /$ $22 \mathrm{~cm} ;$ mur $11: 11 / 13 \mathrm{~cm} \times 15 \mathrm{~cm} ;$ mur $18: 12 \mathrm{~cm} \times 20 /$ $25 \mathrm{~cm} ;$ mur $10: 12 \mathrm{~cm} \times 17 / 20 \mathrm{~cm} ;$ mur $12: 13 / 20 \mathrm{~cm} \times 15 /$ $30 \mathrm{~cm}$. 
1
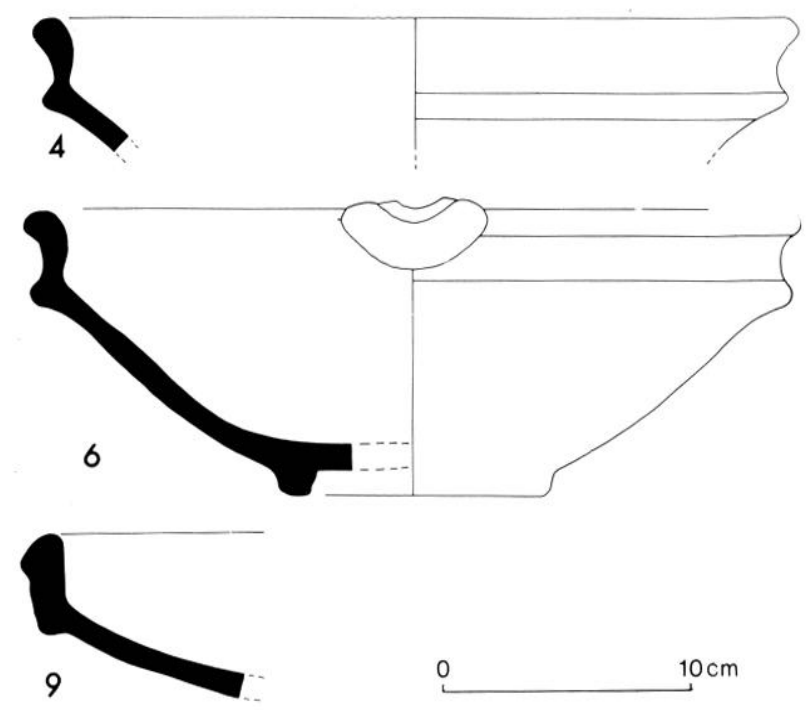

0
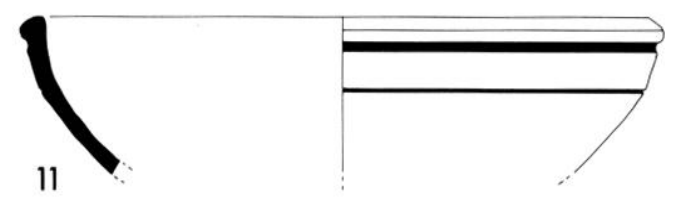

$10 \mathrm{~cm}$
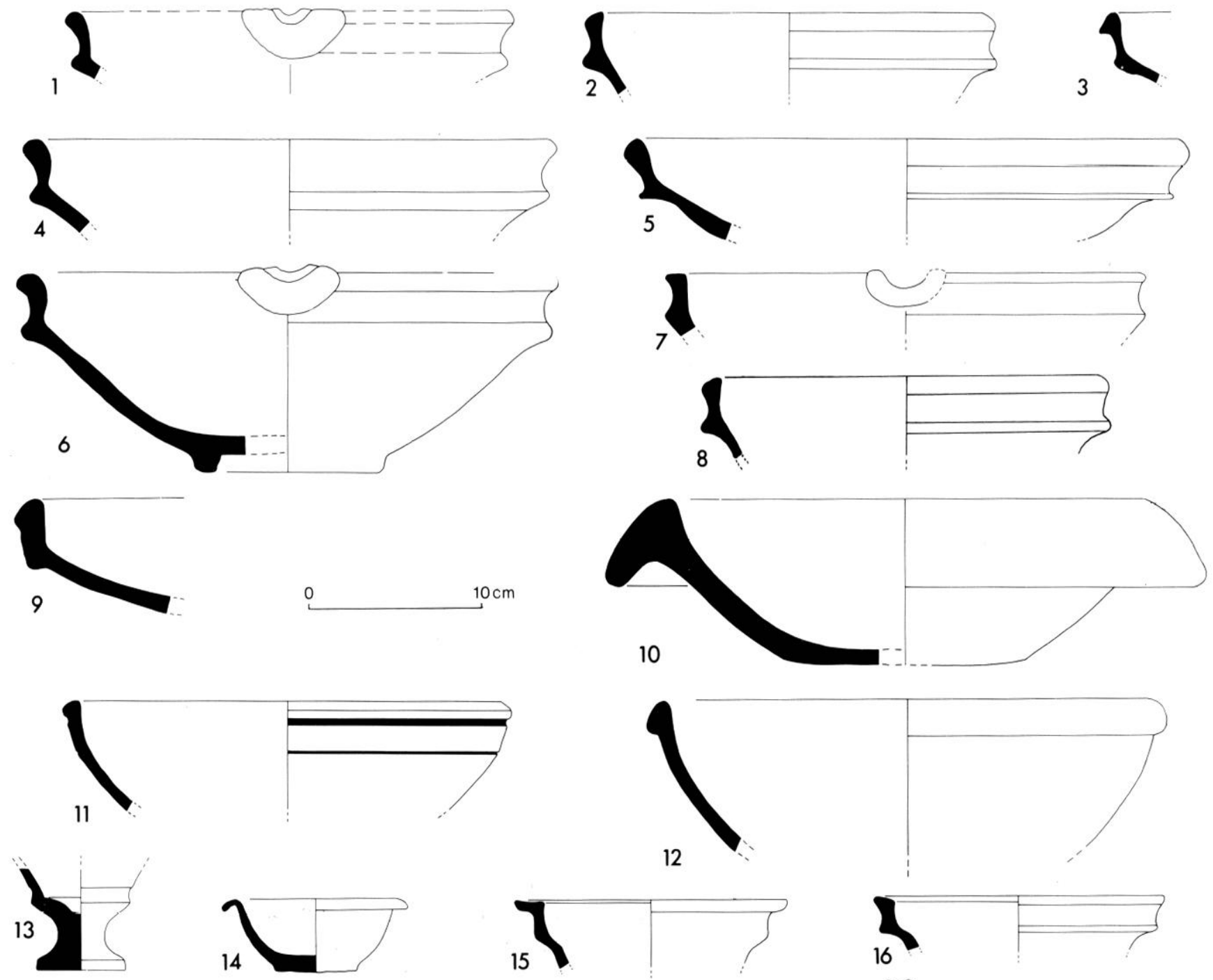

10
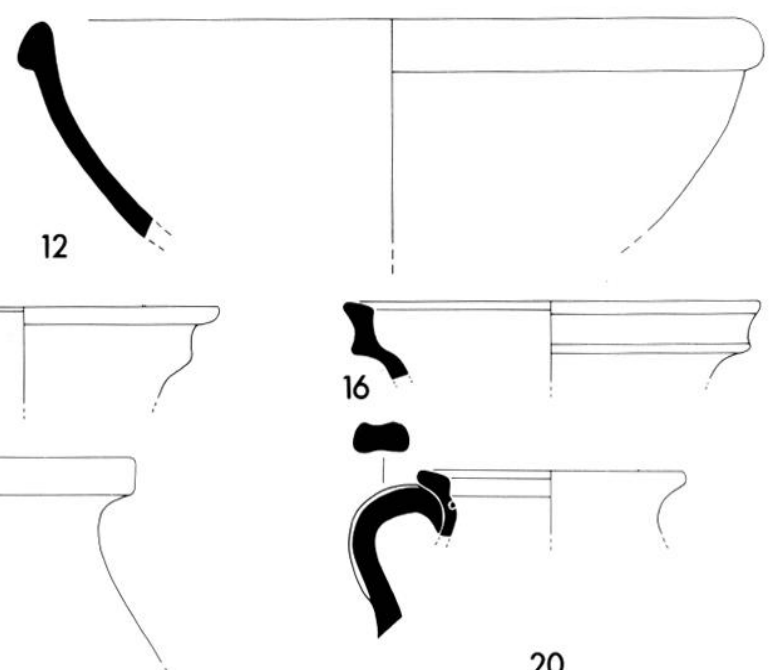

20
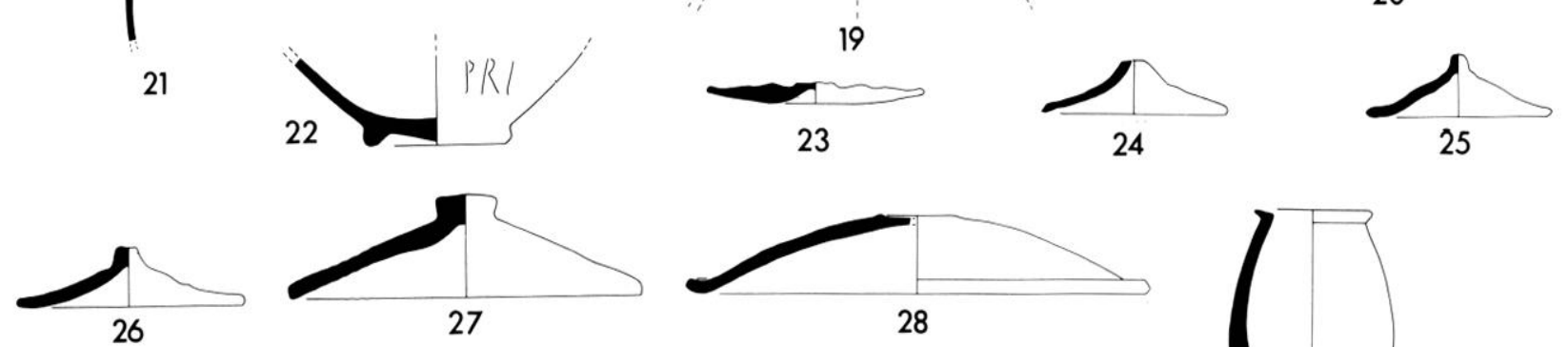

26
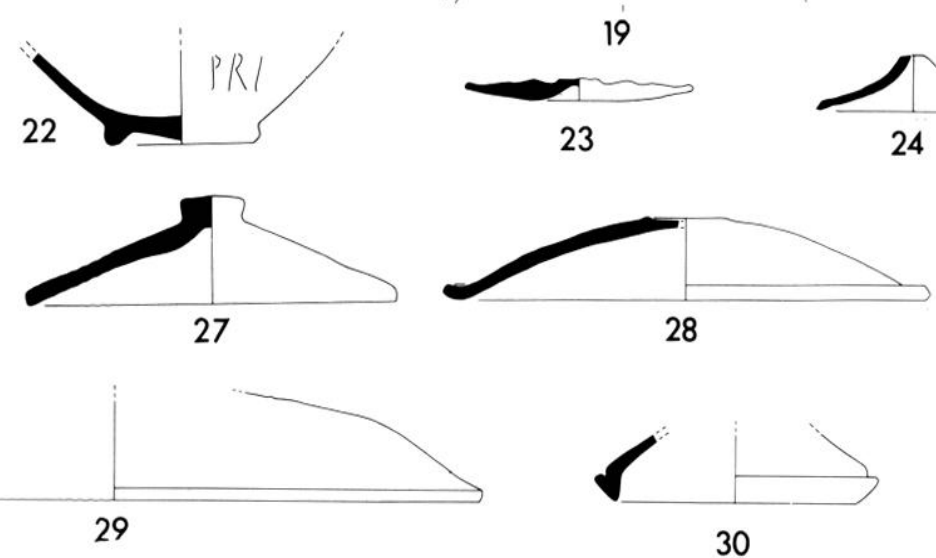

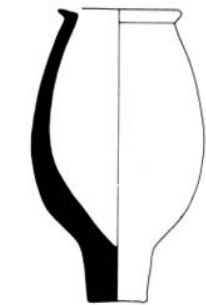

31

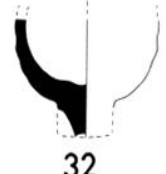

32

Fig. 12 - Céramique commune de l'état I. 
La céramique commune claire, fabriquée probablement en grande partie dans des ateliers locaux, est représentée essentiellement par des formes ouvertes (mortiers, coupes), ainsi que par quelques formes fermées et des couvercles. Des comparaisons ont été effectuées avec la céramique commune claire provenant de sites éludiés à Fréjus : les Aiguières ${ }^{5}$, le Clos de la Tour (Rivet, 1980), les nécropoles de Saint-Lambert et du Pauvadou (Nécropoles de Fréjus, 1985̃), ainsi qu'avec celle de l'épave des Roches d'Aurelles qui transportait des céramiques provenant d'ateliers fréjusiens (Bérato el alii, 1986a). Les datations du matériel de la Porte d'Orée et des autres sites comparés concordent et s'articulent autour du milieu du $\mathrm{I}^{\mathrm{er}} \mathrm{s}$. de notre ère.

\section{Formes ouvertes}

Les mortiers (fig. 12, nos 1 à 10) constituent la série la plus abondante. La pàte, légèrement micacée, varie du beige clair au beige foncé, ou passe à l'orangé selon la cuisson, et contient un dégraissant de grosseur variable. Un type domine, caractérisé par un rebord vertical concave dans sa partie centrale et terminé par deux lèvres plus ou moins épaisses et protubérantes. Un seul exemplaire $\left(n^{\circ} 6\right)$ à panse tronconique et pied annulaire est complet. Trois exemplaires seulement possèdent un bec verseur $\left(n^{\circ s} 1,6\right.$ et 7$)$ : il est probable que les autres, bien qu'incomplets, appartiennent aussi à la catégorie des mortiers ( $n^{\circ} 7$ et $n^{\circ} 4$, similaires aux formes Rivet $39 \mathrm{~b}$ et 41 du Clos de la Tour; $\mathrm{n}^{\circ} 3$ à comparer au type 3A de l'Ormeau [Pasqualini, 1988, p. 160 et fig. 2]). L'exemplaire $n^{\circ} 6$ est très proche d'un mortier trouvé dans l'épave des Roches d'Aurelles (type $5)$, dont le naufrage a été placé dans les années 70 de notre ère. Un deuxième type de mortier est représenté par le no 9 , à rebord vertical (Rivet, forme $39 a$ du Clos de la Tour et $17 \mathrm{c}$ des Aiguières). Le dernier modèle $\left(n^{\circ} 10\right)$ est caractérisé par une lèvre épaisse, un fond plat et une pâte orange clair avec un dégraissant irrégulier (Rivet, type 42 du Clos de la Tour et $15 \mathrm{c}$ des Aiguières).

Les coupes (fig. 12, nos 11 à 14) sont de plusieurs types; la pansc est arrondie, la lèvre à peine marquée $\left(n^{\circ} 11\right)$ ou légèrement pendante $\left(n^{\circ} 12\right)$ (Rivet, 1980 , 13a). Un autre type possède un décor à collerette et une lèvre incurvée, peut-être pour recevoir un couvercle. Une coupe à pied ( $\left.n^{\circ} 13\right)$ type Rivet $38 d$ des

5 I. Rivet, La céramique commune du site de VilleneuveAiguières à Fréjus (manuscrit prèt pour la publication du site des Aiguières) : nous remercions ici l'auteur qui a bien voulu nous permettre de citer cet article inedit.
Aiguières et un petit bol à lèvre pendante et fond plat ( $\left.\mathrm{n}^{\circ} 14\right)$ complètent la série des formes ouvertes.

\section{Formes fermées}

Elles regroupent des cols de grandes cruches ou d'urnes (fig. $12, n^{\text {os }} 15$ à 22), avec des rebords très marqués, une dépression centrale ( ${ }^{\circ} 16$ à comparer avec la forme Rivet 6 des Aiguières) et une lèvre aplatie et en baïonnette ( ${ }^{\circ}{ }^{\text {s }} 18$ et 19 , parallèle au type 2 des Roches d'Aurelles et type B1 de l'Ormeau). Il faut ajouter à cette liste un petit pot ovoïde $\left(\mathrm{n}^{0} 21\right)$ à pâte dure, peut-être d'origine italique. Enfin un fond $\left(n^{\circ} 22\right)$, dont on ne peut dire s'il appartient à une forme fermée ou ouverte, provenant de la pièce 2 , porte sur la base de la panse un graffite : PRI.

Les couvercles (fig. 12, $\mathrm{n}^{\text {os }} 23$ à 30 ) forment une catégorie que l'on peut diviser en quatre types. Le premier $\left(n^{\circ} 23\right)$ est très plat avec une lèvre relevée vers l'extérieur. Le deuxième type (n ${ }^{\text {os }} 24$ à 27) regroupe des couvercles munis d'un bouton de préhension, à paroi plus ou moins rectiligne et lèvre peu marquée. Dans cette série, un couvercle $\left(n^{\circ} 27\right)$ en céramique culinaire africaine (forme Ostia 2, 302) est daté du début du $\mathrm{I}^{\text {er }} \mathrm{s}$. Le troisième type $\left(\mathrm{n}^{\text {os }} 28\right.$ et 29) se distingue par une panse légèrement arrondie, un replat sur le sommet et une lèvre peu marquée vers l'extérieur et une pâte orangée avec un gros dégraissant. Des formes similaires ont été découvertes dans la nécropole du Pauvadou (tombe 32) et dans la tombe 309 de la nécropole de SaintLambert (inédites). Le dernier type de couvercle est incomplet. La lèvre est très relevée vers l'extérieur, sans doute pour s'emboîter dans un récipient ( $\left.n^{\circ} 30\right)$. Deux bouchons d'amphore sont à ajouter à cette liste de couvercles ( $\mathrm{n}^{\text {os }} 31$ et 32 ).

\section{INTERPRÉTATION DES VESTIGES DE L'ÉTAT I}

Ces vestiges se situent dans la portion nordouest du bassin portuaire antique. Les murs de l'état I de la Porte d'Orée sont orientés nord-sud et estouest, de la même manière que les quais méridionaux et orientaux reconnus jusqu'à présent.

Du bassin antique du port on ne connaît que le quai méridional sur lequel a été édifié le monument dit de la Lanterne d'Auguste et une partie des quais est.

Comment replacer les murs de la Porte d'Orée dans cet ensemble portuaire? Un renseignement très précieux est fourni par un plan anonyme de 1825 , où sont mentionnés par un trait rouge "les restes exis- 


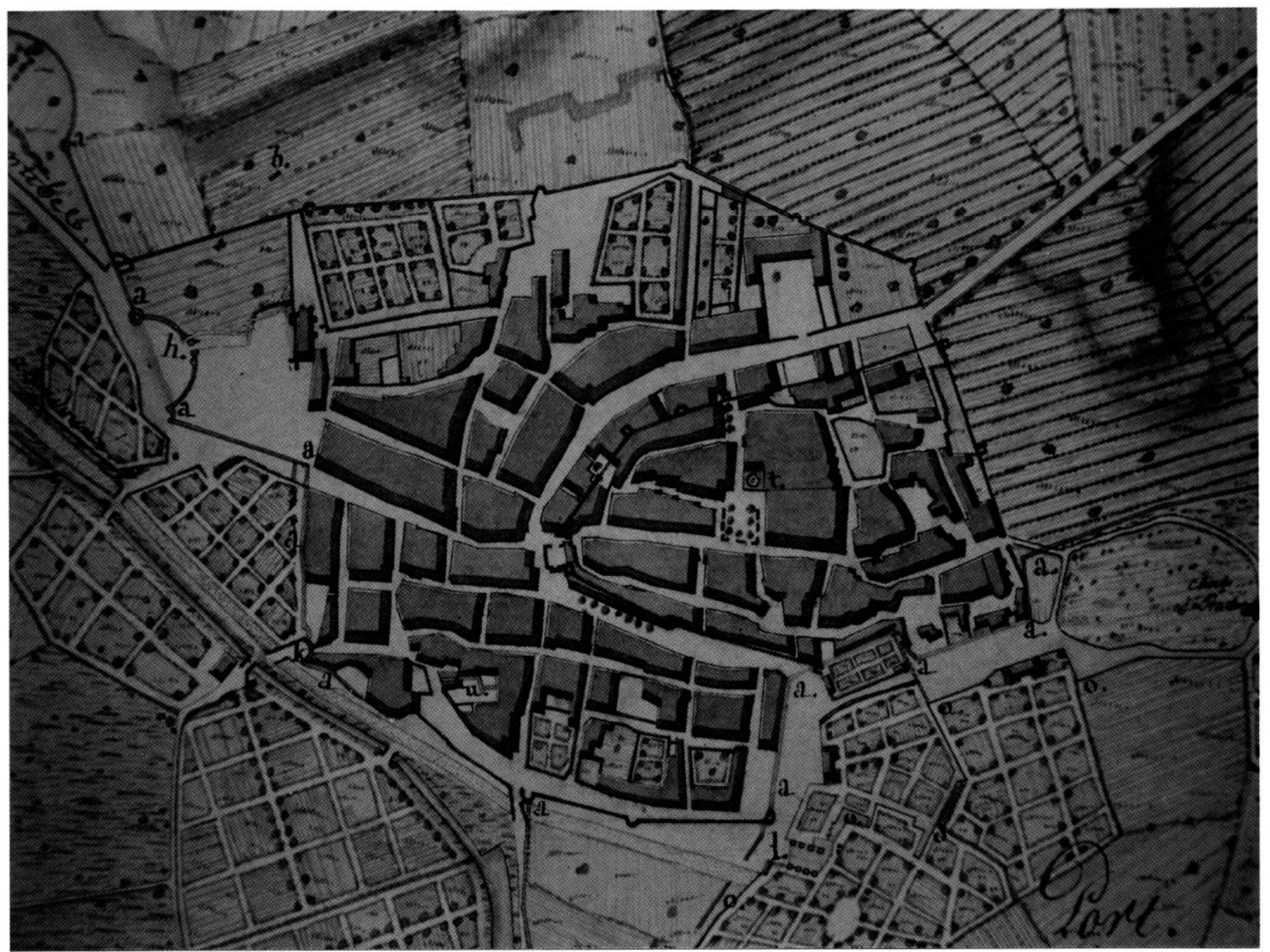

Fig. 13 - Plan de Fréjus de 1825, anonyme.

tants des constructions romaines" ${ }^{6}$ (fig. 13). On voit le tracé du port et des quais et on y retrouve (noté en () sur le plan) le mur 18 de l'état I. Celui-ci sert de limite de parcelle, comme à l'heure actuelle encore : le mur de clôture de propriété est bàti directement sur le mur romain. En revanche, aucune autre structure n'a jamais figuré sur un plan, même ancien. Nous avons là, confirmation que ce mur a été identifié comme faisant partie des structures encore visibles du port antique.

La Porte d'Orée est située vraisemblablement au débouché de la sortie sud de la ville antique qui était établie sur une butte de grès rocheux faisant partie des premiers contreforts de l'Estérel. Le port a donc été aménagé au pied de cette butte, sûrement dans des marais préexistants.

6 Plan de 1825, anonyme, manuscrit conservè à Paris, Bibliothèque nationale. Département des cartes et plans (Réf. GEC: 9309), échelle 1/5000".
Autre élément important à ajouter en faveur de cette identification: il s'agit de la similitude des niveaux NGF entre les murs de l'état I et le quai méridional aux abords de la Lanterne d'Auguste, faisant donc partie d'un même ensemble (fig. 14).

Le niveau de l'eau peut paraître très bas par rapport à la hauteur des quais et pose le problème de la variation du niveau de la mer depuis l'époque romaine $^{7}$ (fig. 15). D.J. Blackman ${ }^{8}$ affirme qu'en

7 Les constructions de l'état I peuvent raisonnablement ètre associèes à l'aménagement des structures portuaires antiques. II n'y a pas incompatibilité entre le niveau de la plus haute arase des murs $8,9,12$ et 18 , celui de la nappe phréatique actuelle et un niveau moyen restitué de la mer au début de la présente ère. L'éloignement par rapport au rivage actuel et l'ennoiement des constructions antiques par la nappe phriatique sont du ressort de la morphodynamique de l'estuaire de l'Argens et de la zone còtière. Les basses terres de la plaine alluviale, au débouché sur le golfe entre les reliefs des Maures et de l'Estérel, sont constituées de sédiments fins. Dans un tel domaine còtier, le comblement de la profonde ria depuis le 


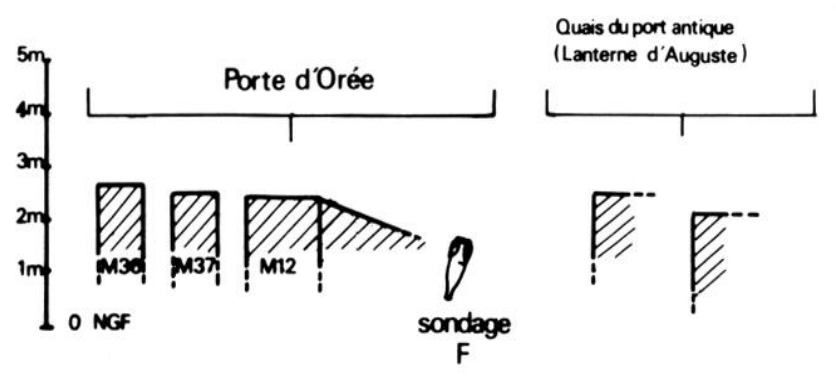

Fig. 14 - Viveaux comparés des quais et structures portuaires connus à Fréjus.

$E$

MOLE

Fig. 15 - Darse de l'état IB : coupe est-ouest.

Médilerranée, durant ces 3000 dernières années, le niveau n'a pas varié de plus de $30 \mathrm{~cm}$, surtout dans nos régions où les mouvements tectoniques des plaques côtières sont faibles. Mais on constate qu'à Portus à Ostie (Testaguzza, 1970, p. 94) dans le port de Claude, il se situe à environ $2 \mathrm{~m}$ en dessous du sommet des quais. En estimant que ce niveau a pu changer de quelques dizaines de centimètres, on

Pliocène ne concerne pas les époques historiques. Par contre. les allerrissements du Quaternaire, de l'Holocène. l'edilication d'un cordon dunaire - qui n'est plus actif aujourd'hui car remplacé par l'aménagement anthropique du littoral - sont bien les sujets d'une étude visant à comprendre les modifications du paysage au cours des 2000 dernières années.

L'examen des photographies aériennes, où l'on distingue nettement les traces d'anciens lits du fleuve et des lagunes littorales totalement comblées ou en voie d'atterrissement, est. convaincant; les ètendues deau isolées de la mer par une flèche littorale (ou lido) construite par la dérive cotière, les divagations des bras du fleuve sont en relation avec l'occupation du sol depuis l'époque romaine jusqu'à nos jours. Le colmatage est bien historique comme en témoignent les vestiges romains reconnus, en l'absence de toutes traces d'occupation humaine antérieure.

Le fait que les ruines romaines, situejes dans la plaine alluviale, soient aujourd hui constamment inondées, témoigne d'un relèvement du toit de la nappe phréatique que l'on peut mettre en relation avec l'éloignement progressif du littoral, consècutif à l'accumulation des sédiments et l'exhaussement. corrélatif de la topographie, qui entrainent une correction du profil d'équilibre des écoulements de la nappe; ceux-ci se font tout naturellement à un niveau plus élevé que lorsque le niveau de base (débouché dans la mer) ètait bien plus rapproché et le colmatage moins avancé (P. Poupet, UPR 290 du C.NRS).

8 Blackman, 1973; - voir en particulier Flemming et alii, 1973, p. 62 et Ports et villes engloutis, 1981. obtient à Fréjus à peu près les mèmes mesures que sur le site de Portus ${ }^{9}$.

Il convient maintenant d'examiner plus en détail l'aménagement et la destination des éléments composant l'ètat $\mathrm{I}$.

\section{L'esplanade}

On peut envisager la construction du mur 37 lors de l'état I, sorte de contrefort servant de mur de soutènement dans ce terrain sableux assez instable et infiltré par la nappe phréatique (sans doute de manière moins importante qu'à l'heure actuelle). L'espace ainsi délimité avec le mur 9 et la pièce 3, remblayé de sable et d'une dimension de $24,50 \mathrm{~m}$, forme une sorte d'esplanade. Par comparaison, le niveau NGF de la maison augustéenne contemporaine de cet état (fouilles de 1988 de la place Formigé) ${ }^{10}$ est de $18,20 \mathrm{~m}$. Le niveau de la Porte d'Orée étant d'environ $2,50 \mathrm{~m}$ la dénivellation entre la ville et le port est de plus de $15 \mathrm{~m}$.

Ce terre-plein, situé à proximité immédiate de la zone portuaire (et probablement au débouché de l'ac:cès sud de la ville), offrait un grand espace et une

9 P.-A. Février a eu l'amabilité de nous communiquer son rapport sur la fouille qu'il a effectuée en juillet 1979, sur une partie du quai nord-est du port, au lieu-dit les Horts; il en ressort une difference de niveau notable avec les structures de la Porte d'Orée. En effet, la partie supérieure du quai varie entre 0,85 et $0,97 \mathrm{~m}$.V(iF. Mais la proximite des restes d'un batiment antique, construit dans le prolongement du quai. peut faire penser à un aménagement différent dans cé secteur opposé du port; d'autre part, on observe sur les photographies de l'auteur les signes de la destruction et de l'arrachement du parement des murs qui a pu affecter aussi l'arase du quai.

10 Niveaux fournis par P.-A. Février. 
surface plane assez importants pour servir d'aire de déchargement immédiat ou de stockage temporaire et de passage, fonction qui s'est perpétuée à travers les ètats du site jusqu'à l'époque médiévale.

\section{Le vide sanitaire (sondage $F$ )}

Lors de l'ouverture de ce sondage contre la face ouest du mur 9, nous avons pu observer que ce terreplein était isolé par un vide sanitaire d'amphores. A l'époque antique, la nappe phréatique était probablement moins haute car directement drainée dans le bassin du port. A Fréjus, d'autres sites d'époque romaine comme les Aiguières ont été retrouvés lors des fouilles noyés par la nappe phréatique (Goudineau, 1982 , p. 280 et 282). Au-dessus de la couche d'amphores étaient disposés, nous l'avons noté plus haut, d'énormes blocs de scories de terre cuite et d'argile rubéfiée : s'agissait-il d'une sorte de radier pour augmenter la stabilité ou le drainage du sol ou simplement d'un comblement quelconque pour le surhausser? Il est difficile de le dire.

Dans le même secteur (T.D), une amphore de type Dr. 7 a été trouvée à une profondeur identique. On peut supposer que ce vide sanitaire s'étendait sous toute l'esplanade; malheureusement faute de temps, le sondage peu profond pratiqué le long du mur 37 (S.E) n'a pas pu nous permettre d'atteindre la couche d'amphores.

\section{La pièce 3}

La pièce 3, comme nous l'avons vu plus haut, est largement ouverte vers le sud et sur l'esplanade. Cette rotonde ou tour devait servir avec le mur 9 (dont elle est la continuité en élévation) de limite ouest au bassin portuaire durant l'état IA. En admettant que l'esplanade puisse être interprétée comme un lieu de circulation et de déchargement, cette pièce circulaire devait avoir une fonction liée à l'activité portuaire ou d'autres activités connexes. En tous cas, il s'agit d'un édifice à vocation publique. Sa structure même (épaisseur des murs, largeur de l'ouverture dépourvue de seuil) permet d'écarter tout rôle défensif à ce bàtiment. En la comparant à d'autres tours connues à Fréjus, des différences de taille apparaissent. La tour est de la Plate-Forme a un diamètre extérieur de $9 \mathrm{~m}$; celle de la porte des Gaules et la tour méridionale de la Butte Saint-Antoine ont chacune $10 \mathrm{~m}$ de diamètre, la plus vaste étant celle de la Porte d'Orée, avec un diamètre de $12 \mathrm{~m}$. Lorsque les bateaux arrivaient de la mer et s'engageaient dans le canal pour atteindre le port, on devait apercevoir à partir de la Lanterne d'Auguste la tour et son esplanade. Dans cette tour, pour la période qui concerne l'état I, plusieurs remblaiements liés à un sol de circulation en argile fortement damée avec un pendage est-ouest ont été observés. La large entrée dont l'enduit blanc est conservé évoque une activité et une circulation intensives.

\section{Le bassin ou darse}

Dans l'état IA, le mur 9 et la pièce 3 servaient de limite à cette esplanade et au-delà, s'étendait un bassin portuaire (fig. 16). Hypothèse qui est renforcée par la présence à ce moment-là du premier plan incliné.

Le mur 18, situé à l'est du site et distant de $36 \mathrm{~m}$ du mur 9 , peut être interprété non comme un quai mais comme une portion d'un môle qui aurait servi à amarrer des embarcations de taille réduite. Un môle de taille similaire existe dans le port de Claude à Ostie : il s'agit du "molo sinistro" construit en blocs de travertin ('T'estaguzza, 1970, p. 91).

L'épaisseur relativement faible de certaines de ces structures portuaires (M9, M11, M12), tout au moins dans ce secteur, peut étonner mais s'explique sans doute par le fait que le port de Fréjus, situé complètement à l'intérieur des terres, ne souffrait ni des tempêtes, ni du ressac marin et n'avait pas besoin de maçonneries importantes pour résister à l'action de la mer.

Dans ce premier ètat, nous pouvons restituer un bassin avec un plan incliné (M8), délimité par un môle (M18), une aire de circulation et de déchargement (esplanade), complétéc par une tour (P3) dont la fonction n'est pas connue. Le quai principal, quant à lui, doit se situer plus au nord, dans la partie non fouillée ou sous la rue A. Briand (fig. 4).

Pour des raisons non déterminées (instabilité de terrain?) les structures de l'état I sont très vite abandonnées; le premier plan incliné (M8) est nivelé, le mur 12 est construit, ce qui a pour effet de rétrécir l'emprise de la darse de $7 \mathrm{~m}$ par rapport au môle M18.

\section{Les plans inclinés de l'état IB}

Deux plans inclinés sont ajoutés au mur 12, lors de l'état IB (fig. 16). Celui du sud (le seul observé dans sa totalité) large de $3 \mathrm{~m}$, mesurait environ $10 \mathrm{~m}$ de long hors d'eau, avec une pente assez forte de 14" (en tenant compte des variations du niveau marin depuis l'époque antique). On peut expliquer cette déclivité par le fait que le bassin, délimité par le môle M18, étant de dimensions assez réduites, la cale ne pouvait pas trop s'avancer dans l'eau en pente douce, car elle aurait gêné la circulation des embarcations dans la darse (fig. 15). Sa surface ne porte pas 

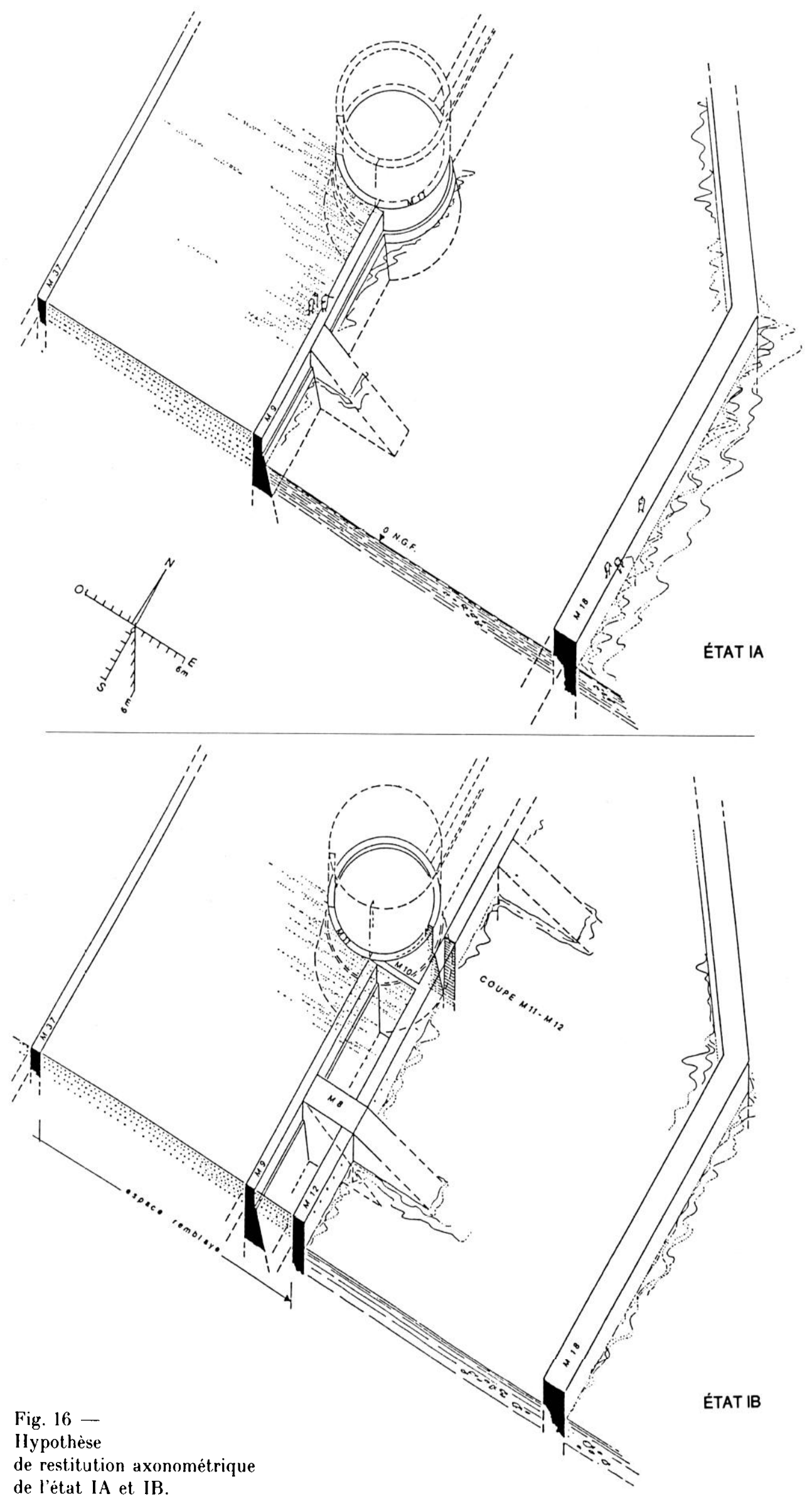
de traces d'usure, mais D.J. Blackman explique que ces plans inclinés pouvaient être pourvus d'aménagements en bois afin de ne point abìmer la quille des bateaux (Blackman, 1982, p. 206). D'ailleurs, dans la partie supérieure du mur 8 , de part et d'autre, subsistaient deux orifices ayant pu servir de point d'ancrage à un revêtement de bois.

La taille relativement réduite de la cale en longueur et en largeur évoque une utilisation par des embarcations de dimensions modestes. Comme élément de comparaison, les deux bateaux (horeia) découverts récemment dans le port de Telo Martius (Toulon) (Borréani el alii, 1987, p. 20-22) sont d'une taille en rapport avec ces aménagements : en effet, ils mesurent respectivement 2 et $3 \mathrm{~m}$ de large et 6 et $8 \mathrm{~m}$ de long. Ces horeia ont été identifiés à Toulon comme des barques de pêche. Autre parallèle, à Portus, dans le port de Claude, des embarcations ont été mises au jour et interprétées aussi comme des bateaux de pêche qui mesuraient $2 \mathrm{~m}$ de large et environ $7 \mathrm{~m}$ de long (Testaguzza, 1970, p. 144). Si les plans inclinés de la Porte d'Orée servaient effectivement de cales à bateaux, on peut envisager qu'elles accueillaient ce type de barque.

D'autres exemples sont connus dans les ports du monde antique : à Rome, sur les quais du port du Tibre à Marmorata des rampes d'accès d'une largeur de $1,80 \mathrm{~m}$ et longues de 7 à $13,30 \mathrm{~m}$ ont été observées, certaines avec des paliers (Le Gall, 1952, p. 195 et pl. XV et XVI). Dans le port de Claude, un plan incliné non couvert mais bordé de parapets débouche sur le côté d'un bâtiment construit sur le môle droit (Testaguzza, 1970, p. 77 et 99). Il mesure un peu moins de $4 \mathrm{~m}$ de large sans les parapets et est conservé sur environ $6 \mathrm{~m}$ de longueur, ce qui le rapprocherait des cales de la Porte d'Orée.

Par ailleurs, on connaît d'autres exemples de type sensiblement différent : ils sont disposés en batterie, avec une pente en général plus faible, dotés d'une couverture, et sont souvent à usage militaire pour remiser hors d'eau les navires de guerre. De tels plans inclinés existaient à Zéa (au Pirée), au Cap Sounion, à Apollonia (Blackman, 1973, p. 126-131), par exemple.

Malgré des structures assez imposantes bâties en appareil soigné (opus vittatum), mises en place durant la première moitié du $\mathrm{I}^{\mathrm{er}} \mathrm{s}$. de notre ère, il apparaît que cet ensemble portuaire n'a fonctionné que quelques dizaines d'années, puis a été comblé dans les années 80 après $\mathbf{J}$.-C. (à l'exception de la rotonde), pour faire place aux aménagements de l'état II, modifiant complètement la fonction du site.

\section{L'ÉTAT II}

Il s'agit d'un ensemble monumental à plan axial, bàti en petit appareil régulier (opus vittatum, module d'environ $12 \times 20 \mathrm{~cm}$ ), lié au mortier comme pour la plupart des monuments de cette époque à Fréjus (fig. 17). L'emprise de ce bâtiment coincide avec celle du bassin antérieur de l'état IB, sur lequel il se greffe en réutilisant certains murs en soubassement.

La limite nord formée par un mur continu (M15, $70 \mathrm{~cm}$ d'épaisseur et $5 \mathrm{~cm}$ de ressaut) qui vient s'appuyer à l'ouest contre le parement extérieur du mur 11 de la rotonde (P3) et s'arrête à l'est avant le mòle M18. Dans un deuxième temps (état IIB), un mur de clôture M1 (50 à $60 \mathrm{~cm}$ d'épaisseur) est rajouté au nord, ainsi qu'un escalier M23 s'appuyant à la fois contre la rotonde et contre le parement externe du mur 15 , dont les joints sont ponctuellement repris lors de cette addition.

La limite est est celle du môle de l'état I, audelà duquel le terrain forme une dénivellation assez importante $(0,56 \mathrm{~m})$. La limite ouest est plus complexe car l'état II réutilise la rotonde P3 mais recouvre les autres murs de l'ètat I (murs $8,9,10$ et 12). En fait, c'est le mur $13(75 \mathrm{~cm}$ de large) qui forme la limite ouest de ce nouveau bâtiment, car la pièce 9 n'est qu'un couloir en cul-de-sac entre la rotonde et lui.

Au sud, la fouille n'a pas été prolongée au-delà de l'emprise du projet initial de parc de stationnement. Mais quelques indices nous permettent d'en extrapoler la limite. Sur le plan de la ville de Fréjus de 1825 cité plus haut (voir supra, p. 174 et fig. 13), il apparait clairement que les vestiges interprétés comme "quai» correspondent à la limite cadastrale actuelle à l'est ainsi qu'au sud, où ils se prolongeraient sous une partie du chemin communal bordant le secteur fouillé. A cet endroit, on note une dénivellation de 1,40 m similaire à celle remarquée à l'est, et les murs antiques sont encore visibles en plusieurs points. Nous pouvons donc penser que la limite sud du site est matérialisée par cette dénivellation ou terrasse, tout comme le môle 18 forme sa limite est.

\section{LES SALLES ET ESPACES}

Cet ensemble est composé de huit salles ou espaces (P4 à 7,9 à 12) et réutilise également un bâtiment: la rotonde ou tour P3 liée à l'état I (fig. 18). Le plan s'organise autour d'une pièce centrale $\mathrm{P} 5$ flanquée symétriquement de deux salles : à l'est (P6) et à l'ouest (P4), ouvrant au sud sur un portique à colonnade P11, lui-même contigu à un 


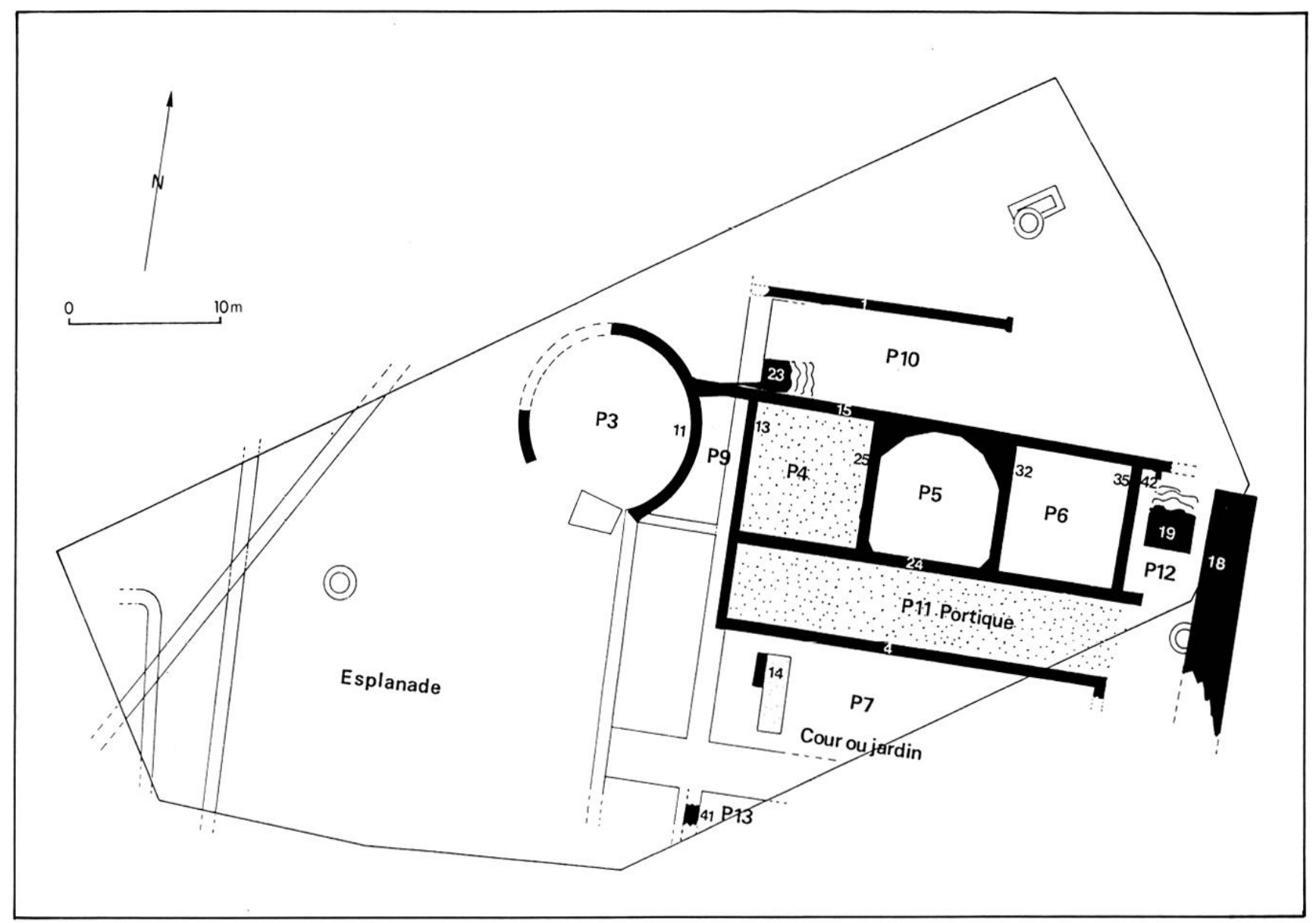

Fig. 17 - Plan sélectif de l'état II.

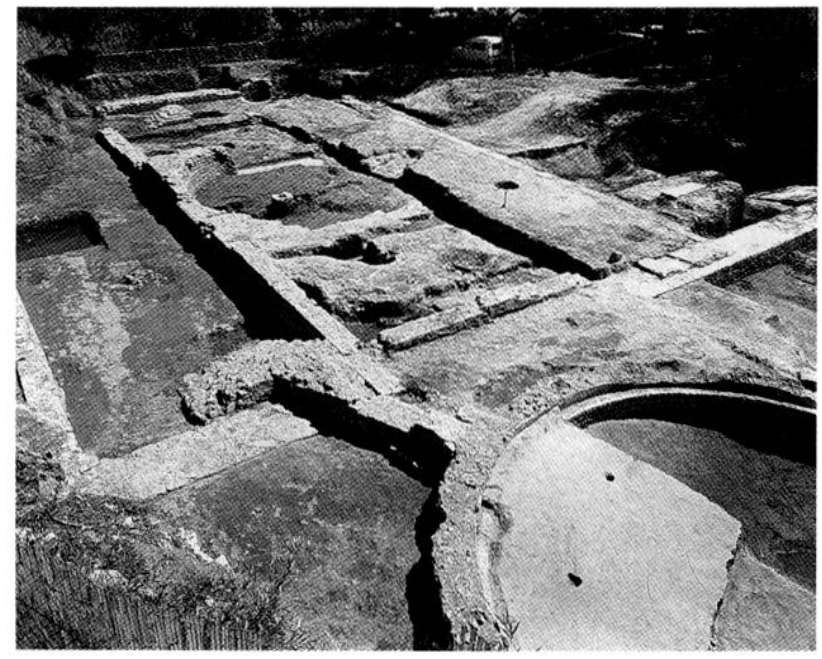

Fig. 18 - Vue d'ensemble des structures composant l'état II.

espace ouvert P7 de plain-pied avec l'esplanade déjà existante à l'état précédent.

Sur le côté est, un passage P12, communiquant avec le portique 11 au sud et avec la pièce 10 au nord par un escalier M19, est délimité par l'ancien môle
M18 servant à présent de bordure au nouveau bàtiment. Nous n'avons retrouvé aucune trace de cloisonnement sur M18, aussi nous est-il impossible de préciser si ce dégagement était fermé à l'est et servait de couloir ou s'il ne consistait qu'en une galerie semi-abritée.

Un muret M1, terminé par un piédroit, clôture au nord des pièces 4,5 et 6 un long espace rectangulaire P10 desservi à l'ouest par l'escalier M23, et rejoint le passage P12. Le mur 1 est bâti directement. sur le plan incliné nord de l'état IB, ce qui explique son aspect irrégulier et sa pente accentuée vers l'est : une rangée de tuiles plates est utilisée pour tenter de redresser le lit de pose.

La salle centrale $P 5$ mesure $9,30 \mathrm{~m}$ de large sur $9 \mathrm{~m}$ de long et se termine au nord par une abside à pans coupés et au sud par deux segments d'ares de cercle. Les deux autres pièces $\mathrm{P} 4$ et $\mathrm{P} 6$ la flanquent symétriquement et mesurent $8 \mathrm{~m}$ sur $9 \mathrm{~m}$. La pièce P4 a gardé un sol en béton de tuileau (posé sur un épais remblai de sable); il est accroché aux ressauts internes des murs nord, est et ouest. Le mur nord (M15) des pièces P4 et P5 est percé d'ouvertures 


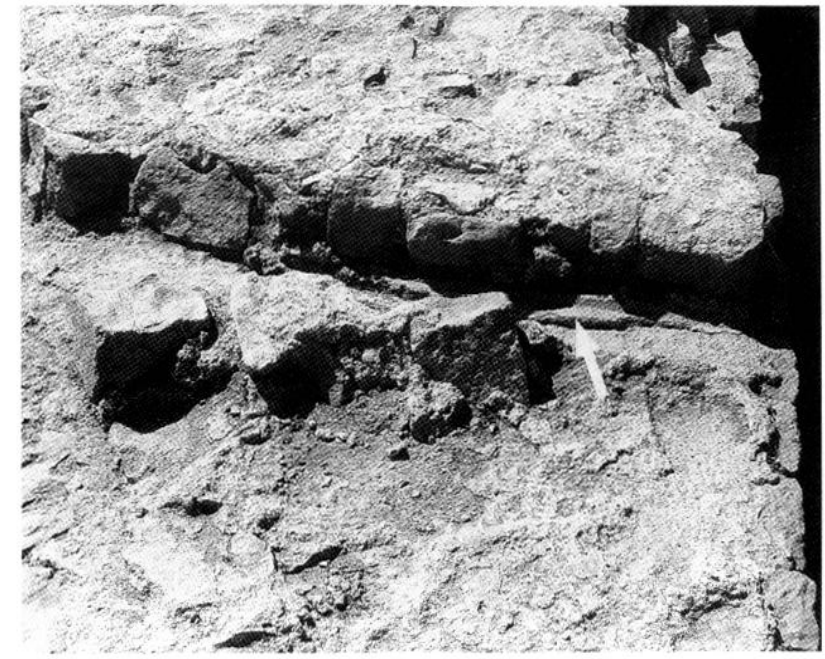

Fig. 19 - Espace P5) : empreinte d'un tuyau de plomb dans le mur 15.

espacées à intervalles réguliers (quatre dans chaque mur) et situées au même niveau, où l'on distingue encore l'empreinte d'un tuyau de plomb d'un diamètre de $15 \mathrm{~cm}$ environ (fig. 19) ayant servi probablement à une arrivée d'eau. Ln fragment de tuyau a d'ailleurs été retrouvé dans le dépotoir de l'ètat IV adjacent à la pièce P10. Le béton encore en place dans la pièce $\mathrm{P} 4$ bouche partiellement une de ces ouvertures $^{11}$. En P6, le mur M15 était trop arasé pour que l'on puisse y discerner des ouvertures de mème nature.

Le portique P11, de forme rectangulaire $(25 \mathrm{~m}$ de long sur $4,50 \mathrm{~m}$ de large), est délimité par un stylobate de $75 \mathrm{~cm}$ de large sur les côtés ouest (M13) et sud (M4) : on distingue nettement la trace d'arrachement d'environ vingt-deux blocs du stylobate. Le mur M13 se réduit à une fondation de mortier (fig. 20) de la largeur du stylobate, reposant directement sur un mur de l'état IB (M12). Aucun mur de clôture n'a été retrouvé à l'est, mais le stylobate retourne en formant un angle droit vers l'espace P7, dont il définit la limite; cependant, il ne porte plus trace de l'arrachement des blocs du stylobate et marque peut-être la fin du portique. Ln sol de béton

11 L'empreinte laissée par les tuyaux de plomb dans le mortier des orifices traversant le mur 15 est un argument important pour les distinguer de simples trous de boulin. Le sol de béton accroché au ressaut de la pièce 4 est peut-être un deuxième état de l'aménagement de l'ensemble: il est aussi possible que les orifices dans cette pièce n'aient jamais fonctionné. Rien dans la pièce 5 ne permet de placer le sol originel au niveau du ressaut interne de l'abside; les murs perpendiculaires avaient été récupérés trop profondément pour que l'on puisse y discerner un ressaut similaire.

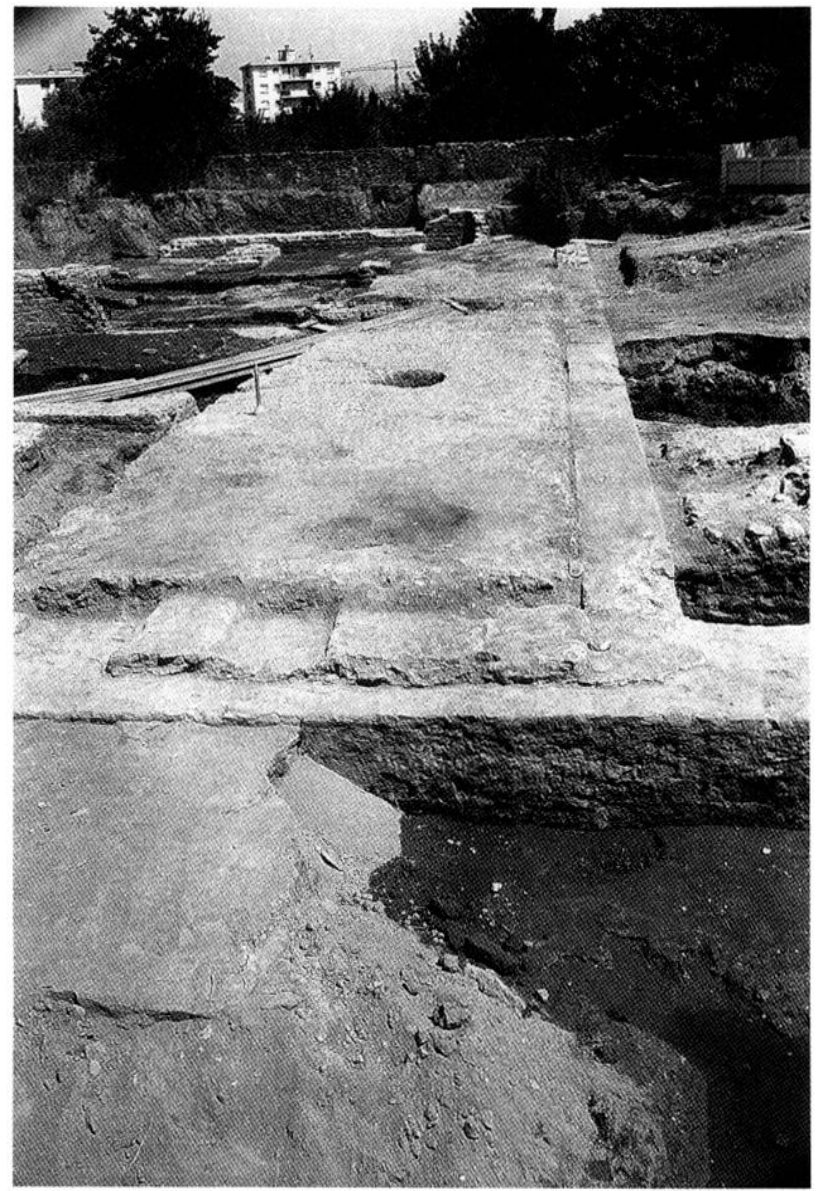

Fig. 20 - Empreinte des blocs stylobates dans le mortier sur le mur 4.

de tuileau est conservé sur presque toute la surface de P11. Il reposait, comme en P4, sur un épais remblai de sable. Ce choix de mode de construction d'un béton posé directement sur du sable, sans radier de pierre intermédiaire, n'est pas étonnant, car ce type de remblai est très porteur.

On peut donc imaginer que le portique communiquait avec le couloir ou passage P12, d'où l'escalier M19 menait directement à la pièce P10. Il est possible que ce passage vers Pl0 ait été ménagé au détriment du mur M15, si l'on en juge par la structure $\mathrm{M} 42$, qui est rajoutée perpendiculairement au mur principal : on aurait donc affaire ici à un état IIB lié à la construction de la pièce P10 et de l'escalier M23, dont le niveau de circulation situé plus bas n'a pas été reconnu. Par ailleurs, les joints sur l'escalier M19 sont repris au fer, sauf sur sa partie supérieure où le mortier est très usé. Ce procédé serait plus tardif (Février, 1956b, p. 165 et 181).

Il nous est impossible de déterminer le mode d'ouverture ayant existé entre les pièces $\mathrm{P} 4, \mathrm{P} 5, \mathrm{P} 6$ et le portique : le mur 24 de façade a été presque 
entièrement récupéré à l'époque tardive (état IVB), en dehors de deux chicots de maçonnerie situés en P6 et P12. Ce mur est construit d'un seul tenant, comme son parallèle au nord (M15). Nous sommes cependant tentées de restituer de larges ouvertures vers le portique et vers la mer, liées à la fonction monumentale et publique du bâtiment.

L'espace déterminé par P7 et P13 n'est délimité matériellement que sur deux côtés : au nord, par le stylobate M4, à l'est par son retour. La fouille ayant été limitée dans son extension par le projet de parc de stationnement, nous ne pouvons dire si cet espace se prolongeait jusqu'au chemin communal, ou si un autre élément pouvait s'y intercaler. A l'ouest, un massif de béton de tuileau, dans lequel était placée une marche de grès rectangulaire (M14) portant des traces d'usure sur la moitié ouest de sa surface et un trou de louve (indiquant la présence d'un second élément : marche ou socle?), constitue une limite du côté de l'esplanade. Seul un fragment de mur M41, posé directement sur le mur M12 de l'état IB et aligné avec le mur M13 de la pièce $\mathrm{P} 4$, témoigne d'une clôture probablement partielle de P7/P13 à l'ouest. L'accès au monument devait donc s'effectuer par l'esplanade à travers le portique P11, ou par l'espace. occupé par P7/P13, qui semblerait avoir été une cour ou un jardin, comme le laisseraient penser les fonds d'amphores lestés de cailloux et les trous remplis de terre noire, en place dans la couche $4^{12}$.

La rotonde P3 de l'état I a été conservée et restaurée à l'état II comme en témoigne une reprise de l'enduit intérieur, avec l'application d'un second revêtement. Le système de couverture est soit renforcé, soit modifié, car huit trous de poteaux carrés ou rectangulaires ont été retrouvés, alignés à $1,50 \mathrm{~m}$ du mur 11, à intervalles réguliers (fig. 21). Certains présentaient un calage de tuiles et leur section était de $20 \mathrm{~cm}$ environ. Ce bâtiment ouvrait sans doute encore de plain-pied sur l'esplanade.

\section{LE VIDE SANITAIRE D'AMPHORES DE L'ESPACE P7-P13}

Construit à la place occupée auparavant par le bassin de l'état IB, le monument de l'état II constitue donc une extension de l'espace urbain au détriment d'un aménagement portuaire. Mais ce gain n'a

12 I)es exemples de traces de plantations en pot ou en pleine terre ont été reconnus à Pompéi (Jashemski, 1975); la profondeur moyenne conservée de ces dépôts à la Porte d'Orée varie entre 20 et $60 \mathrm{~cm}$, par rapport au sol de béton du péristyle.

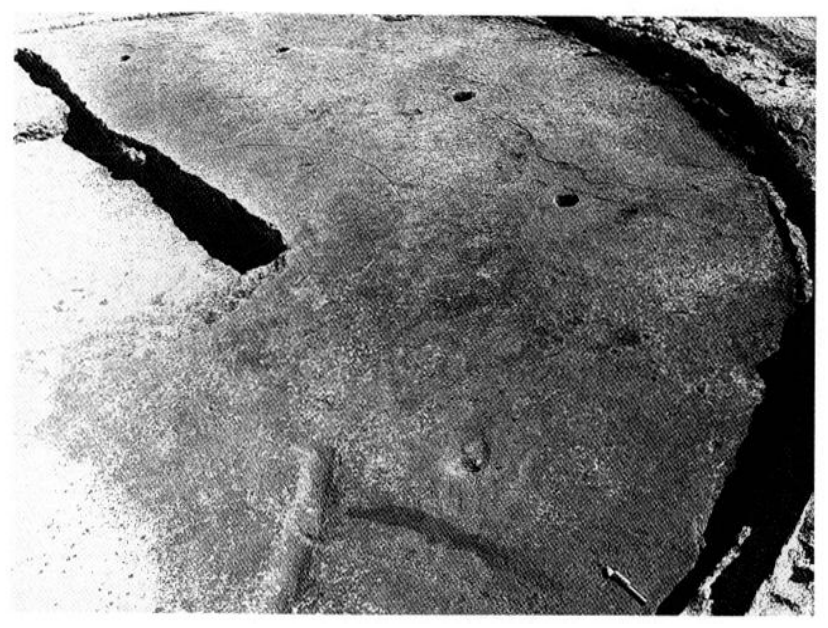

Fig. 21 - Rotonde P3, surface 9 : trous de poteaux disposés parallèlement au mur 11 .

pas été obtenu facilement et, nous le constaterons plus loin, n'a pas subsisté très longtemps dans sa fonction originelle. La raison principale de cette brièveté semble liée au choix même du terrain remblayé, instable par sa nature, malgré tous les efforts déployés pour son assèchement et malgré des fondations de murs très profondes ${ }^{13}$.

La solution adoptée pour tenter de pallier l'instabilité et l'humidité du terrain a été celle d'un comblement homogène de l'ancienne darse par un épais remblai de sable très pur, après construction en élévation des murs constituant le monument de l'état II ${ }^{14}$. A trois reprises, nous avons pu constater l'emploi, par les constructeurs de cet ensemble, d'une technique d'assainissement déjà utilisée à la Porte d'Orée pour l'état IA (sondages D et F) : le vide sanitaire. Les emplacements où nous avons pu effectuer des sondages plus profonds ont révélé deux types de vides sanitaires.

Un sondage ouvert dans la pièce 5 a démontré l'existence, sous une couche de sable, d'un épais remblai de pierres aux arêtes vives, ressemblant à des déchets de taille, à une profondeur de 1,70 m NGF.

Dans une partie de P7, la fouille a permis la découverte, à une profondeur de $2,10 \mathrm{~m} \mathrm{NGF}$, d'un vide sanitaire composé de deux couches d'amphores

13 Deux sondages profonds ouverts en Pl2 ont montre que les murs descendent a $-1,50 \mathrm{~m}$ NGF au moins.

14 Aucune tranchée de fondation n'a été reconnue en fouille : les couches de remblai et d'occupation venaient buter directement contre les structures; l'appareil reste régulier et soigné jusque dans les fondations. Il n'y a que dans la pièce 5 , couche 9, mur 25, que l'on a pu constater l'utilisation d'un module de pierres légèrement plus important et taillé moins soigneusement, ainsi que la reprise au mortier des joints. 
au moins, de type Dr. 2/4 essentiellement, posées côte à côte suivant un pendage nord-ouest/sud-est, parfois tête-bêche, parfois emboîtées partiellement (fig. 22). Pour une superficie de $16,50 \mathrm{~m}^{2}$ environ, fouillée sur une profondeur de $1 \mathrm{~m}$ (jusqu'à la nappe phréatique), nous avons retrouvé soixante amphores au minimum dont vingt-sept entières. Ces amphores sont essentiellement du type Dr. 2/4 avec quelques exemplaires de Pompéi VII ${ }^{15}$. Elles étaient noyées dans un épais remblai de sable (couches 5 et 6 ) et venaient buter contre le stylobate M4.

Au sud, le plan incliné M8 était simplement recouvert par le remblai de sable puisqu'il constituait le seul point ferme de cette zone. Mais le vide sanitaire reprend à partir de $\mathrm{P} 13$ : ici les amphores sont disposées de la même manière qu'auparavant, dans une couche de sable très pur (couche 6 ). Nous avons dégagé une superficie de $4,50 \mathrm{~m}^{2}$, à une profondeur de $2,10 \mathrm{~m} \mathrm{NGF}$, où dix-sept amphores au minimum ont été mises au jour, dont sept presque entières; les Dr. 2/4 sont encore nombreuses et deux amphores de fabrication fréjusienne sont timbrées $M A R I$ (fig. 23).

S'agit-il d'un vide sanitaire ou du moyen de stabiliser un sol humide el mouvant? Le procédé a déjà èté employé à Fréjus, dans un contexte similaire de terrain sableux et très humide à cause de la présence de la nappe phréatique, sur le site des Aiguières, pour une période un peu plus ancienne (Goudineau, 1982, p. 286).

\section{Chronologie de L'ÉTAT II}

Les éléments de datation pour la mise en place de cet état sont fournis par le comblement du bassin de l'état IB : les couches d'occupation ont été largement détruites par les réutilisations successives du site (états III et IV). Hormis les sols de béton de tuileau encore en place dans la pièce $\mathrm{P} 4$ et le péristyle P11, le sol de cour ou de jardin P7, celui de l'esplanade et de la rotonde P3, aucun autre sol n'a survécu.

\section{Stratigraphie de la pièce 3}

Les couches d'occupation dans la rotonde P3 durant l'état I correspondent, nous l'avons vu plus

15 En ce qui concerne l'étude des amphores composant le vide sanitaire de $\mathrm{P} 7 / \mathrm{P} 13$, dont les Dr. 2/4 semblent représenter une production fréjusienne inédite d'après les analyses de M. Picon (1989), cf. l'article de F. Laubenheimer, infra, p. 229265.

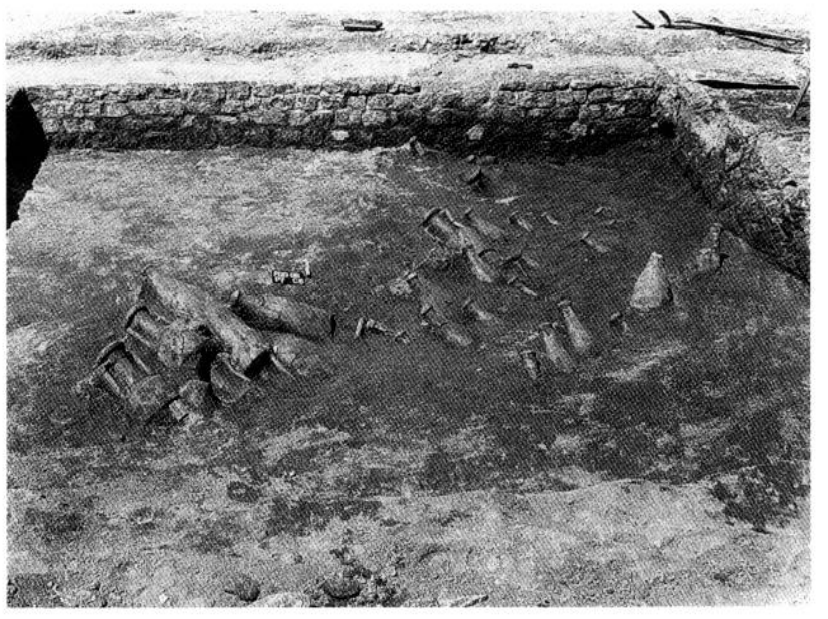

Fig. 22 - Espace P7 : le vide sanitaire d'amphores.

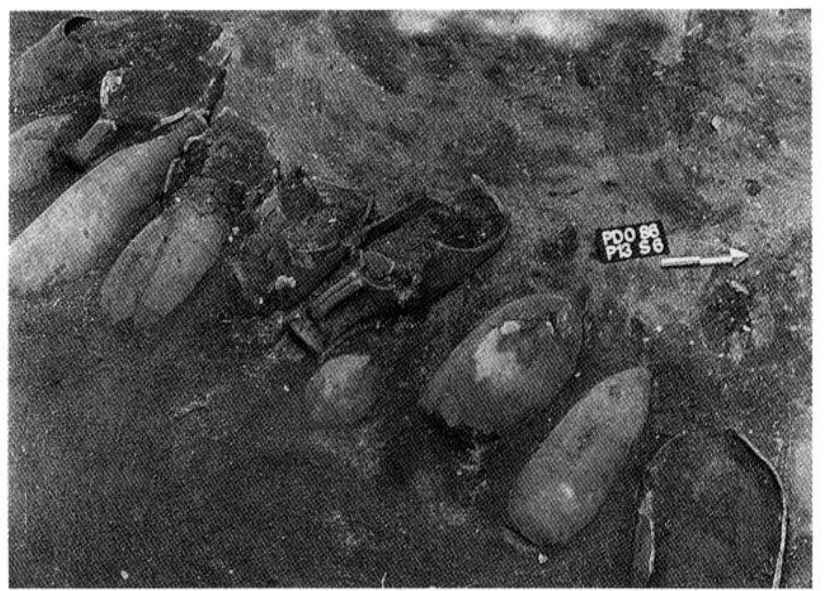

Fig. 23 - Espace P13 : le vide sanitaire d'amphores.

haut, à une série de remblais et à une surface d'occupation. De la même manière, une seule surface soutenue par un remblai (couches 10 et 9) marque la réutilisation de la rotonde à l'état II (fig. 8).

- La couche 10 est constituée par un remblai de sable fin recouvrant le ressaut du mur 11 sur une épaisseur de $20 \mathrm{~cm}$ à l'ouest et de $10 \mathrm{~cm}$ à l'est.

- La surface 9 est un sol d'argile jaune épais de $10 \mathrm{~cm}$ environ, à fort pendage est-ouest, se terminant en bourrelet contre le mur M11. Une réfection de l'enduit et la mise en place de poteaux correspondent à l'utilisation de cette surface.

Le matériel trouvé en couche était peu abondant: la couche 10 contenait une majorité d'amphores $(47,9 \%)$ et de céramique commune à pâte claire locale $(29,1 \%)$. Cette proportion est inversée pour la couche $9(58 \%$ de céramique commune locale et $16,8 \%$ d'amphores). Cette céramique est généralement attribuable au $\mathrm{I}^{\mathrm{er}} \mathrm{s}$. après $\mathrm{J}$.-C. 


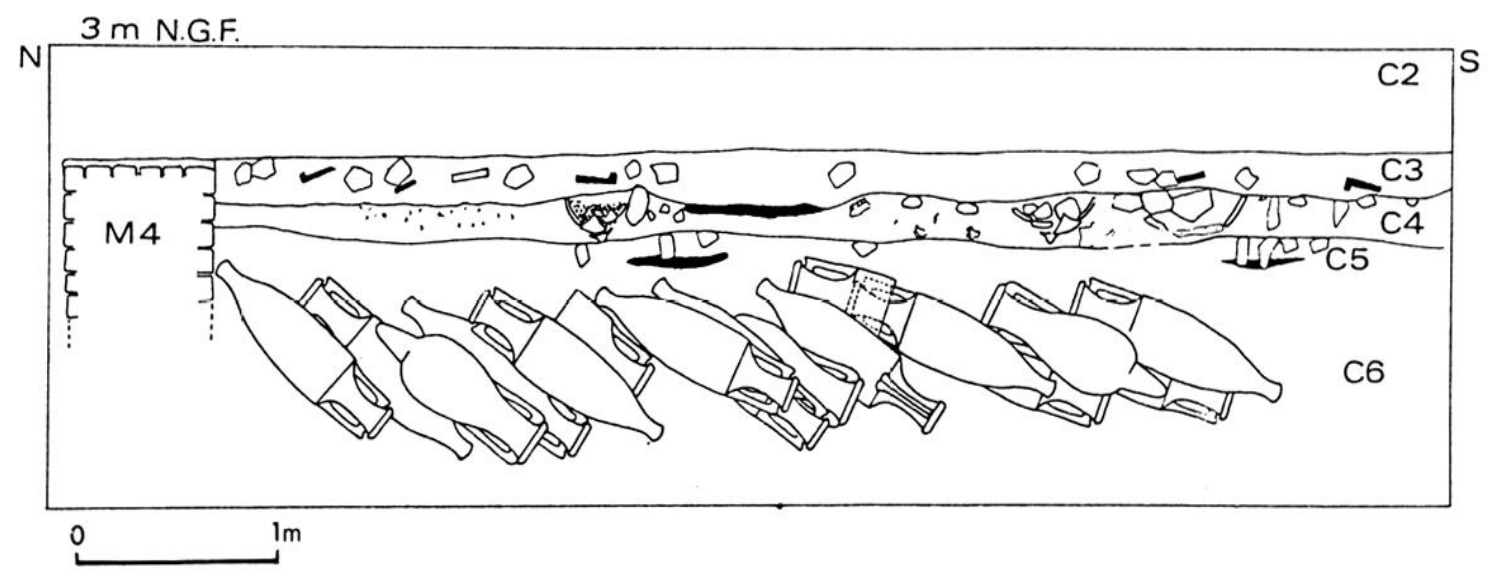

Fig. 24 - Espace P7 : coupe stratigraphique nord/sud.

\section{L'espace P7-P13}

La mise en place du vide sanitaire d'amphores (couches 5 et 6 ) et d'une surface aménagée dans un espace ouvert (couche 4) forme l'essentiel de la stratigraphie pour cette période en P7 (fig. 24).

Le matériel provenant de la couche 4 comprend une grande partie d'amphores $(52,5 \%)$, datable du $\mathrm{I}^{\mathrm{er}} \mathrm{s}$. de notre ère. Les types d'amphores représentés (Gauloise 5, Dr. 2/4 ou Pascual 1, Tarraconaise, Dr. 20 et Richborough 527) (fig. 25), la céramique sigillée de La Graufesenque (formes Drag. 15/17, 18/31, 24/25, 27 et Ritt. 12) associée à l'arétine (un fragment décoré), ainsi que la culinaire africaine du type Hayes 23 ancien ${ }^{16}$, nous permettent d'avancer une datation du dernier quart du $\mathrm{I}^{\mathrm{er}} \mathrm{s}$. après J.-C. $(80$ à 100).

Cette datation est confirmée par le contenu des couches 5 et 6 où, mises à part les amphores du vide sanitaire qui elles-mêmes ne dépassent pas le $\mathrm{I}^{\mathrm{er}} \mathrm{s}$. de notre ère, le matériel datable (sigillée de La Graufesenque, céramiques à paroi fine) est d'époque flavienne.

La superficie fouillée en P13 (fig. 26) était proportionnellement plus restreinte et le matériel (excepté les amphores du vide sanitaire) peu abondant. Sur les quelques tessons retrouvés dans la couche 6 , on compte trois fragments de sigillée de La Graufesenque (Drag. 23 et 27), de la céramique grise de Vaison et des fragments d'amphores (Gauloise 8 et Tarraconaise). La couche 7 ne contient que quelques tessons de céramique grise de Vaison. Ce matériel nous permet d'avancer une datation du dernier quart du $\mathrm{I}^{\mathrm{er}} \mathrm{s}$. après J.-C., qui corrobore celle des couches correspondantes en P7.

16 Type ancien trouvé dans les fouilles de la Place Formigé, inédit : renseignement communiqué par $\mathrm{L}$. Rivet que nous remercions ici.

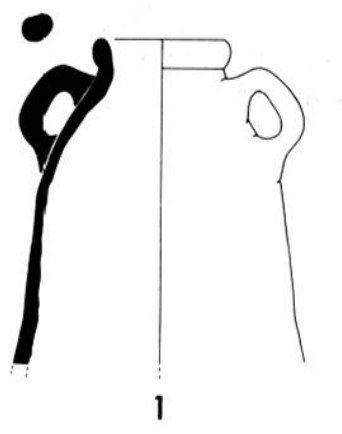

0 $10 \mathrm{~cm}$

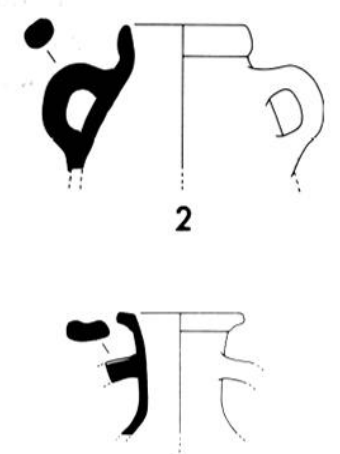

3
Fig. 25 - Espace P7, C4 : amphores de l'état II.

\section{La pièce 5}

Nous avons mentionné plus haut l'emploi d'un remblai de pierres comme vide sanitaire ou pour améliorer le drainage dans cette pièce (voir supra, p. 182) : au-dessus, une couche de sable d'une épaisseur de $20 \mathrm{~cm}$ environ (couche 10) témoignait encore de la nature du comblement du bassin de l'état IB, semblable en cela à celui présent sous les sols de béton de tuileau encore préservés. Ce remblai entamé par les réoccupations successives (états III et IV) contenait du matériel qui n'a été reconnu que dans un sondage profond de dimensions modestes. Il est composé en majorité d'amphores $(58 \%)$ du $\mathrm{J}^{\mathrm{er}} \mathrm{s}$. après $J_{.-C .}$; les autres catégories se répartissent à peu près également, avec cependant un pourcentage un peu plus élevé de sigillées sud-gauloise et arétine $(9,8 \%)$, de céramique modelée $(7,3 \%)$ et de céramique engobée locale $(6,2 \%)$. La date attribuable à cet ensemble est la seconde moitié du $\mathrm{I}^{\mathrm{er}} \mathrm{s}$. après J.-C., confirmée par quatre marques sur sigillée de La Graufesenque, dont trois de la seconde moitié du I ${ }^{\text {er }} \mathrm{s}$. 


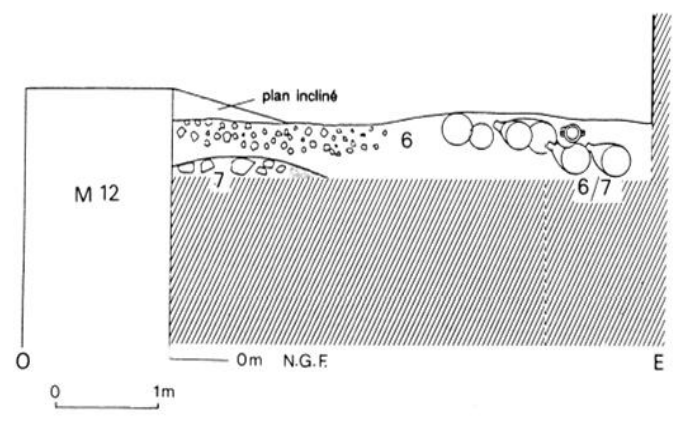

Fig. 26 - Espace P13 : coupe stratigraphique ouest/est.

La couche 9 de la pièce 5 , qui sera étudiée en particulier dans l'état III, fournit un terminus post quem à l'utilisation originelle du bâtiment : début du $\mathrm{II}^{\mathrm{e}} \mathrm{s}$. de notre ère.

\section{LA CÉRAMIQUE COMMUNE}

Fig. 27 et tabl. II, p. 217.

La céramique issue de l'état II est donc représentée essentiellement par des amphores et de la céramique commune. Celle-ci est en grande majorité de fabrication locale, fréjusienne très probablement; on distingue aussi une proportion de céramique italique plus importante que durant l'état précédent, mais qui ne concurrence jamais la céramique locale, bien que quelques formes y trouvent une filiation.

Sans vouloir faire une étude exhaustive, nous avons choisi quelques formes représentatives de la production locale et importee, dominées en grande partie par les formes ouvertes (mortiers, grandes coupes), puis par les urnes. La céramique de Vaison, non présentée ici, est essentiellement composée de cruches, avec quelques formes d'urnes. Les pâtes locales sont toujours jaunes, pouvant tirer sur l'orangé sclon la cuisson (notons une forme fermée en céramique grise de Fréjus). Les pâtes importées, généralement d'Italie, sont brunes ou rouge brun, parfois beige. On note quelques exemplaires de céramique commune sombre de la vallée du Rhône.

\section{Céramiques communes claires locales}

\section{Formes ouvertes}

On reconnaît deux types de mortier, dont l'intérieur est lisse (fig. $27, n^{\text {os }} 1$ à 6 ) :
1 coupe tronconique évasée munie d'un rebord et d'un bec verseur façonné séparément; surplomb au niveau de la carène (Rivet, à paraitre, type 17c).

2 mortier à panse évasée, double lèvre dont la plus grande est arrondie vers l'extérieur et le bas (manque le bec verseur) (Rivet, à paraître, type $16 \mathrm{~b}$, mais sans sablage intérieur).

Deux types de coupes sont également identifiables :

3 grande coupe à lèvre en amande tournée vers l'intérieur (Pasqualini, 1988, type 6A ; Rivet, 1980, type $13 \mathrm{a})$.

4 et 5 grandes coupes à lèvre arrondie ; le pied annulaire est à rattacher à cette forme (Nin, De Luca, 1987, phase 2 , état $4, n^{\circ} 16$ ).

6 coupe à bord en baïonnette, panse à tendance rectiligne, lèvre en méplat oblique (Rivet, à paraître, type 26b).

\section{Formes fermées}

Un type d'urne et de nombreux couvercles de grandes dimensions sont individualisables durant cet état (fig. 27, nos 7 à 10) :

7 grande urne à lèvre triangulaire; ce type est attesté dans les nécropoles de Fréjus, à Saint-Lambert, tombe $87 n^{0} 1$, dimensions similaires mais pâte grise et au Pauvadou/Lagon Bleu, tombe $37 \mathrm{n}^{\circ} 1$, inédite (Béraud, Gébara, 1986, p. 206-207).

8 couvercle très évasé, lèvre triangulaire.

9 coupe ayant peut-être servi de couvercle, forme conique (Bérato el alii, 1985, type 8).

10 bol (?) à lèvre en double bourrelet extérieur, panse globulaire; pâte beige rosé. Provenance inconnue, peut-être locale.

\section{Céramique commune grise locale}

11 col de cruche en calice, pâte grise tendre, fabrication locale (fig. 27, $\mathrm{n}^{\circ} 11$ ) (Rivet, 1980, céramique grise $\left.\mathrm{n}^{0} 24\right)$.

\section{Céramique importée}

Fig. 27, nos 12 à 14 .

12 pot ovoïde, céramique commune sombre de la vallée du Rhône (Desbat et alii, 1979, p. 1-17).

13 urne en céramique brune italique; bord angulaire (Cosa, K-ware) (Dyson, 1976, PD54).

14 couvercle en céramique à pàte beige orangé fine, bord en baïonnette. 

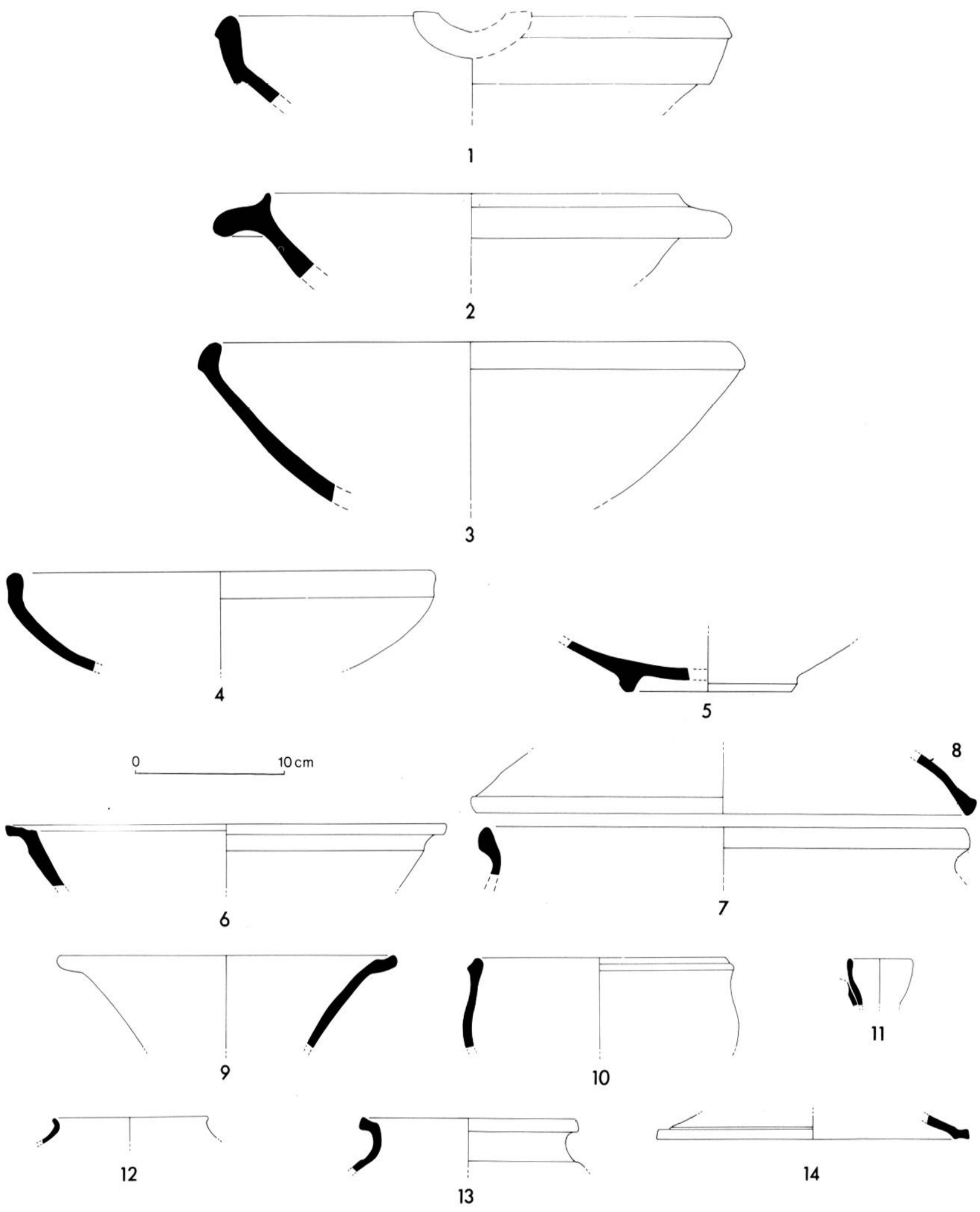

Fig. 27 - Céramique commune de l'état II. 


\section{ESSAI D'INTERPRÉTATION DES VESTIGES DE L'ÉTAT II}

La fonction exacte de ce monument est difficile à déterminer, du fait des réoccupations successives qui l'ont dépouillé très rapidement. Nous pouvons cependant tenter de cerner son utilisation à travers certains indices architecturaux et en le replaçant dans le contexte de la ville antique.

Cet ensemble qui couvre une superficie minimale de $1500 \mathrm{~m}^{2}$, est situé au pied de la ville antique, au sud, dans le prolongement théorique du cardo maximus. Rien n'est connu de l'accès sud de la ville, mais l'importante différence de niveau entre les constructions situées au sommet de la butte de grès et le bassin portuaire laisse envisager un traitement particulier des problèmes d'accès.

A l'époque flavienne, sur ce terrain gagné sur l'emprise du port et situé dans un emplacement privilégié, on construit un bâtiment ouvrant au sud par un portique et un jardin (ou une placette) et faisant face directement au canal d'accès artificiel reliant la mer au port. Ce bâtiment est presque équidistant des extrémités est et ouest du bassin, flanquées des sites majeurs de la Butte Saint-Antoine et de la PlateForme qui le dominent. Le nouveau monument est directement accessible par la ville grâce à l'esplanade (conservée depuis l'état I) qui lui est reliée de plainpied à travers le portique et le jardin (ou placette). La rotonde P3 qui marquait l'emplacement du bassin de l'état I est probablement masquée par ce nouveau bâtiment.

Quelques indices peuvent laisser imaginer sa décoration : tesselles de mosaïque polychrome, fragments d'enduits peints (et enduit blanc encore en place contre le mur 13 dans la pièce P9), placages et fragments de moulures et d'inscriptions très mutilées en marbre (dont une inscription monumentale), attestent d'un aménagement luxueux. Le plan luimême nous oriente vers un bâtiment public : la disposition symétrique autour d'un élément central, la division tripartite précédée d'un portique et d'un espace ouvert, ne sont pas très répandues dans le monde romain (fig. 28). Il faut ajouter à cela l'alimentation en eau probable d'une pièce au moins du monument (la pièce centrale à abside). Seuls certains nymphées de tradition hellénistique rappelleraient. de loin il est vrai, ce plan avec son aspect simultanément imposant et décoratif, inséré en contrebas de la

Fig. $28-$

Hypothèse de restitution du monument de l'état II.

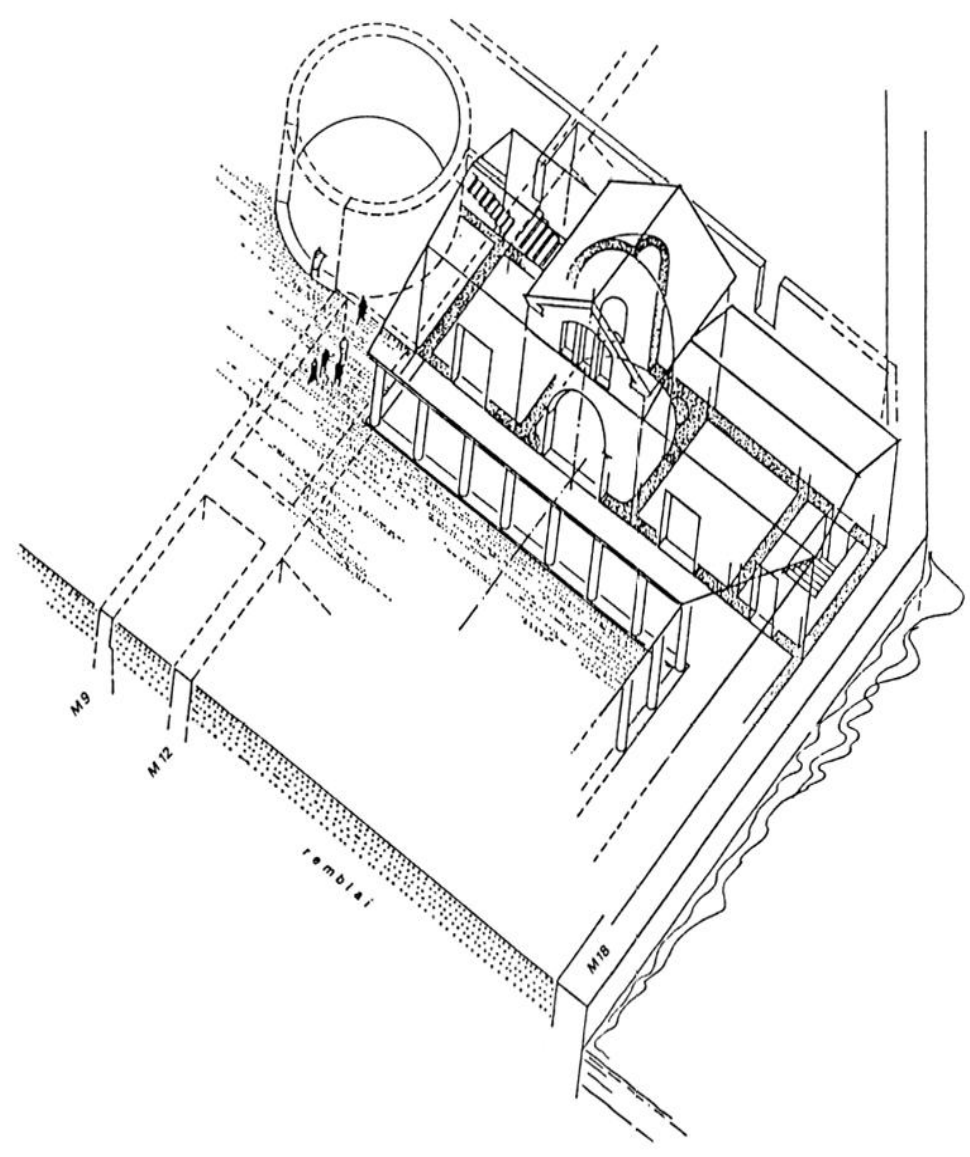




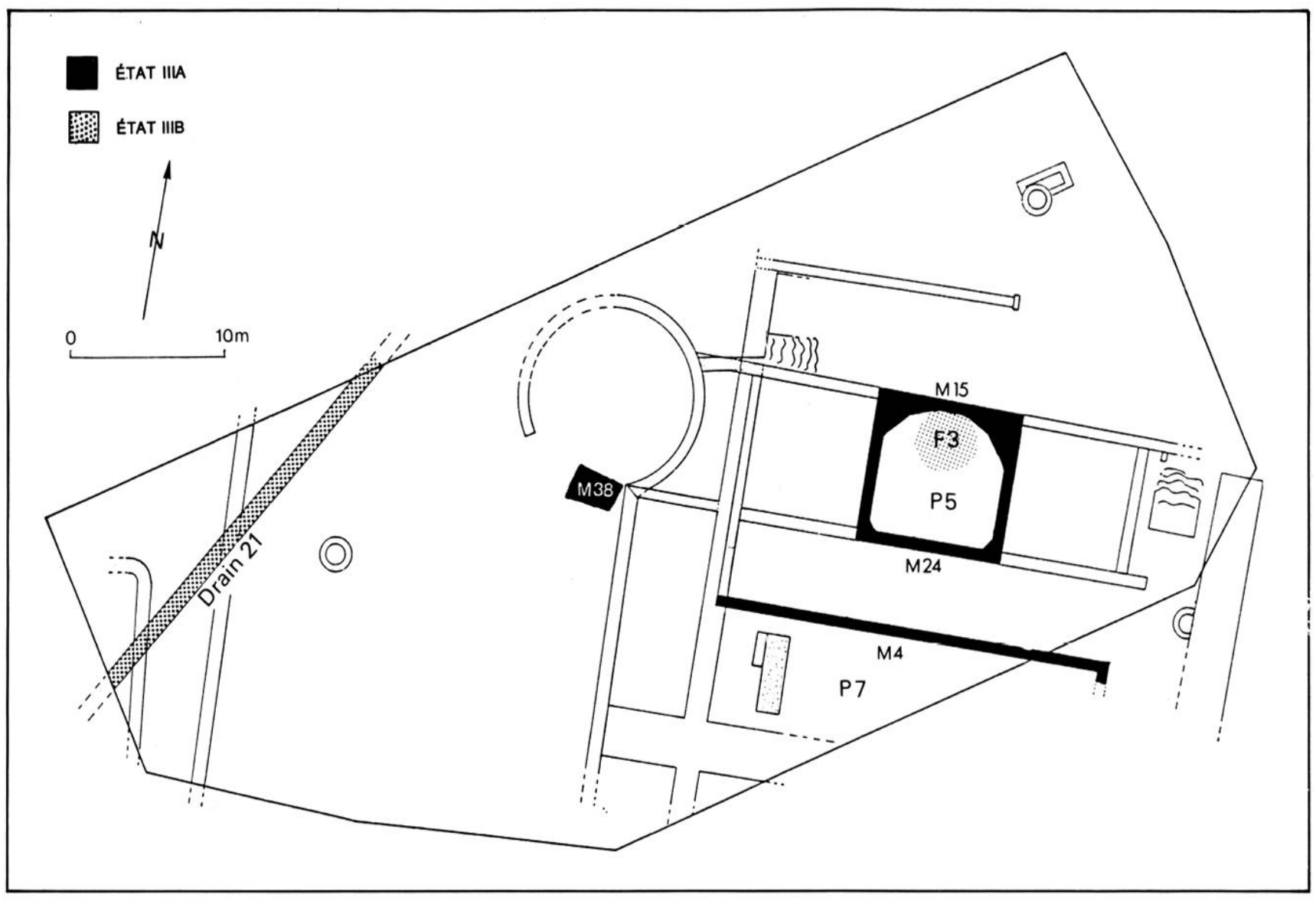

Fig. 29 - Plan sélectif de l'état III.

trame urbaine, en liaison avec le plan d'eau du port ${ }^{17}$.

Ce monument public à caractère somptuaire situé à l'orée de la ville n'aura vécu, en raison même du choix d'un terrain difficile, que quelques décennies. Sa construction est à mettre en rapport avec le développement urbain de l'époque flavienne.

\section{L'ÉTAT III}

Le bâtiment d'époque flavienne de l'état II a èté utilisé comme monument public jusque vers le début du $11^{\circ} \mathrm{s}$. de notre ère. Ce terminus post quem est. confirmé par la datation des couches d'occupation correspondant à l'état IIIA et B (fig. 29).

17 Veuerburg, 1965, et notamment les $n^{0 *} 28,29,31$, 32, 37, 38 et 39 (Palestrina, Tivoli); - Aupert, 1974, nymphèes de type $C, F$ ou ( $i$; pour des parallèles mème lointains, voir Chemtou, Cherchell, Hippone; -- Glaser, 1983, paralleles avec Ialysos, Sikyon, Phigaleia, Épidaure, Perachora, Tenos, Argos: - Lavagne, 1988.
L état IIIA est caractérisé par une réoccupation des structures de l'état II, encore certainement intactes en élévation, mais probablement déjà dépecées au moins partiellement de leurs revêtements muraux et de sols. En effet, les couches correspondant à l'état IIIA contiennent de nombreux fragments d'enduit, de tesselles de mosaïque, de placages ou de moulures de marbre. Dans un deuxième temps (état IIIB), un important remblai vient recouvrir la partie ouest du site.

\section{ÉTAT IIIA : LA RÉOCCUPATION DE L'ÉTAT II}

Elle est discernable dans la piece 5 el l'espace 7 (fig. 30). Le sol de la pièce j̀ est d'abord détruit, puis remblaye (couche 9) et une vaste fosse 133 de diamètre approximatif de $2,40 \mathrm{~m}$ est aménagée contre le parement interne de l'abside. I)ans cette fosse (fig. 31), hormis le matériel fragmentaire (60\% d'amphores et $16,8 \%$ de sigillée), nous avons retrouvé un dolium écrasé en place, bordé sur le côté est 


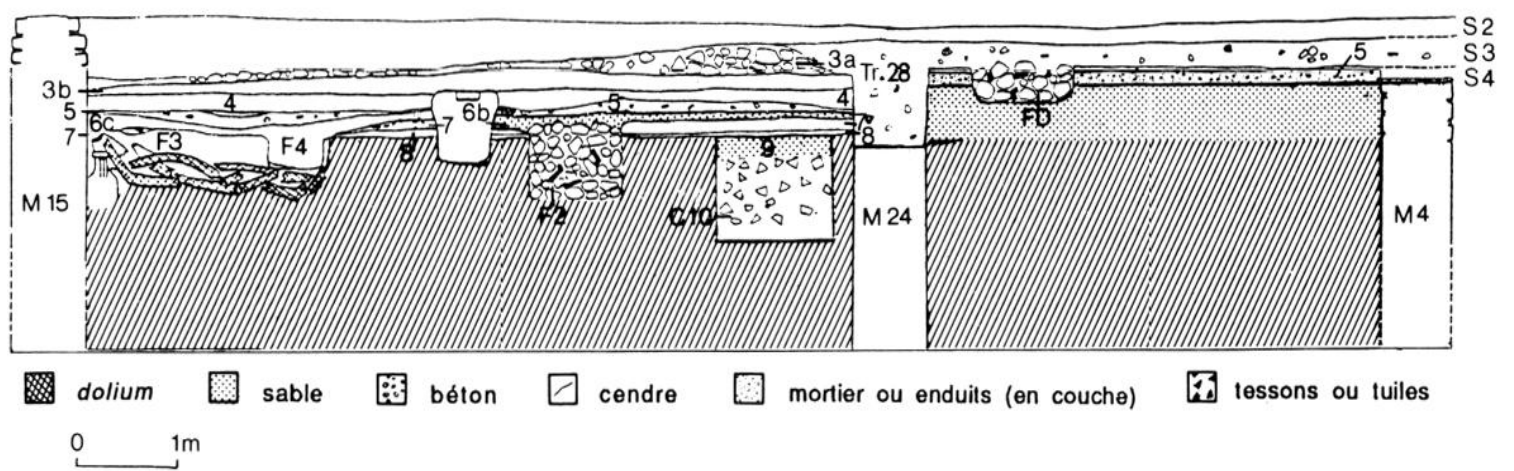

Fig. 30 - Pièces P5 et P11 : coupe stratigraphique nord/sud.

par quatre amphores: l'une à pâte fréjusienne du type Lenzbourg, l'autre à lèvre à double inflexion externe (Laubenheimer, 1991, p. 253); (fig. 32); une Dr. 2/4 locale et une amphore de Tarraconaise estampillée FVS (marque inédite, fig. 33, $\mathrm{n}^{\circ} 1$ ).

Cet ensemble fosse $3 /$ surface 9 , datable de la première moitié du $\mathrm{II}^{\circ} \mathrm{s}$. de notre ère, est rapidement recouvert par une couche de remblai (couche 8) contenant une majorité de tessons d'amphores $(56 \%$ de Dr. 20, Dr. 2/4 ou Pascual 1, Tarraconaise, Gauloise), une assez grande quantité de céramique commune claire $(8,4 \%)$ et de céramique modelée et culinaire $(8 \%)$, locales. La sigillée de La Graufesenque est présente $(5,3 \%)$ et fournit les éléments de datation de la première moitié du $\mathrm{II}^{\mathrm{P}} \mathrm{s}$.

La couche 7 est une couche d'habitat percée irrégulièrement d'une série de fosses ( $\mathrm{F} 3 \mathrm{~B} 1,3 \mathrm{~B} 2$, $3 B 3,4)$ et possédant un foyer (foyer $\left(C_{1}\right)$ : on perçoit encore le comblement de la grande fosse 3 (couche 9) fait de fragments d'amphore, de tuiles et de pierres. La fosse $3 \mathrm{BI}$ est circulaire (diamètre : $60 \mathrm{~cm}$ ) et remplie de détritus (coquillages, os d'animaux, tessons, fragments de tuiles, de verre, de fer et d'enduit peint, ainsi qu'une grande quantité de charbon de bois).

Les fosses $3 \mathrm{~B} 2$ (profondeur : $25 \mathrm{~cm}$; diamètre : $80 \mathrm{~cm}$ ) et $3 \mathrm{B3}$ (profondeur : $30 \mathrm{~cm}$; diamètre : $60,5 \mathrm{~cm})$, circulaires, etaient remplies de terre noire avec de grosses pierres au fond. La fosse 4, également circulaire (diamètre supérieur: $60 \mathrm{~cm}$; diamètre inférieur : $50 \mathrm{~cm}$; profondeur : $35 \mathrm{~cm}$ ) était creusée dans le comblement de la fosse 3 (couche 9) et ne contenait que quelques ossements animaux, des tessons et un rebut de cendre au fond.

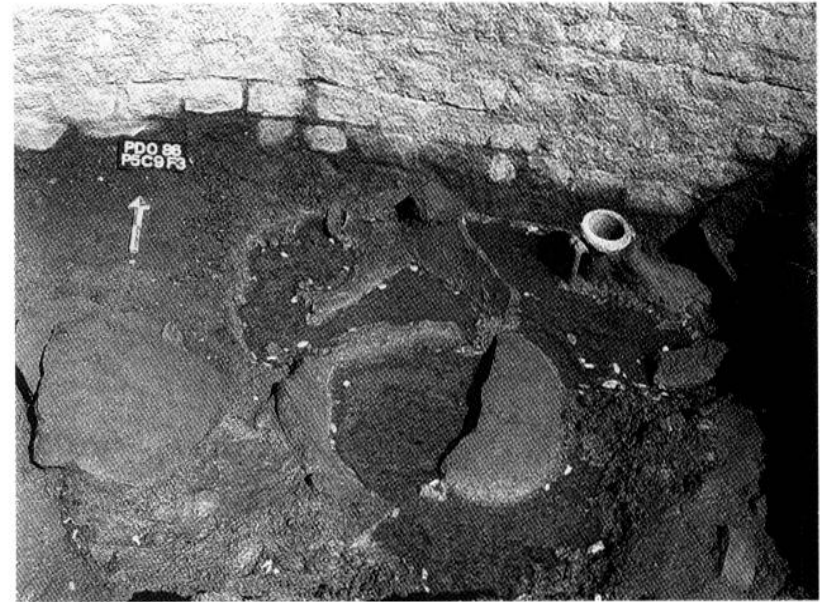

Fig. 31 - Pièce P5, fosse $3:$ dolium et amphores en place.
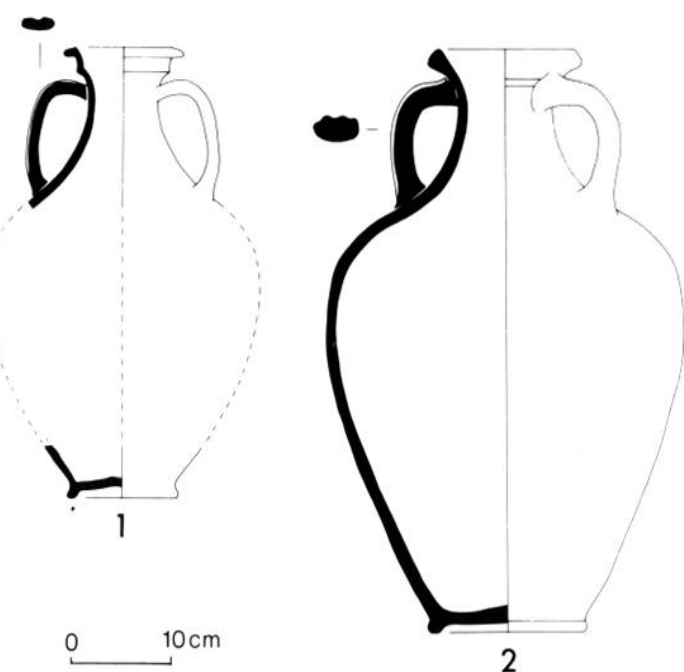

Fig. 32 - Pièce P5) : amphores gauloises de la fosse 3. 

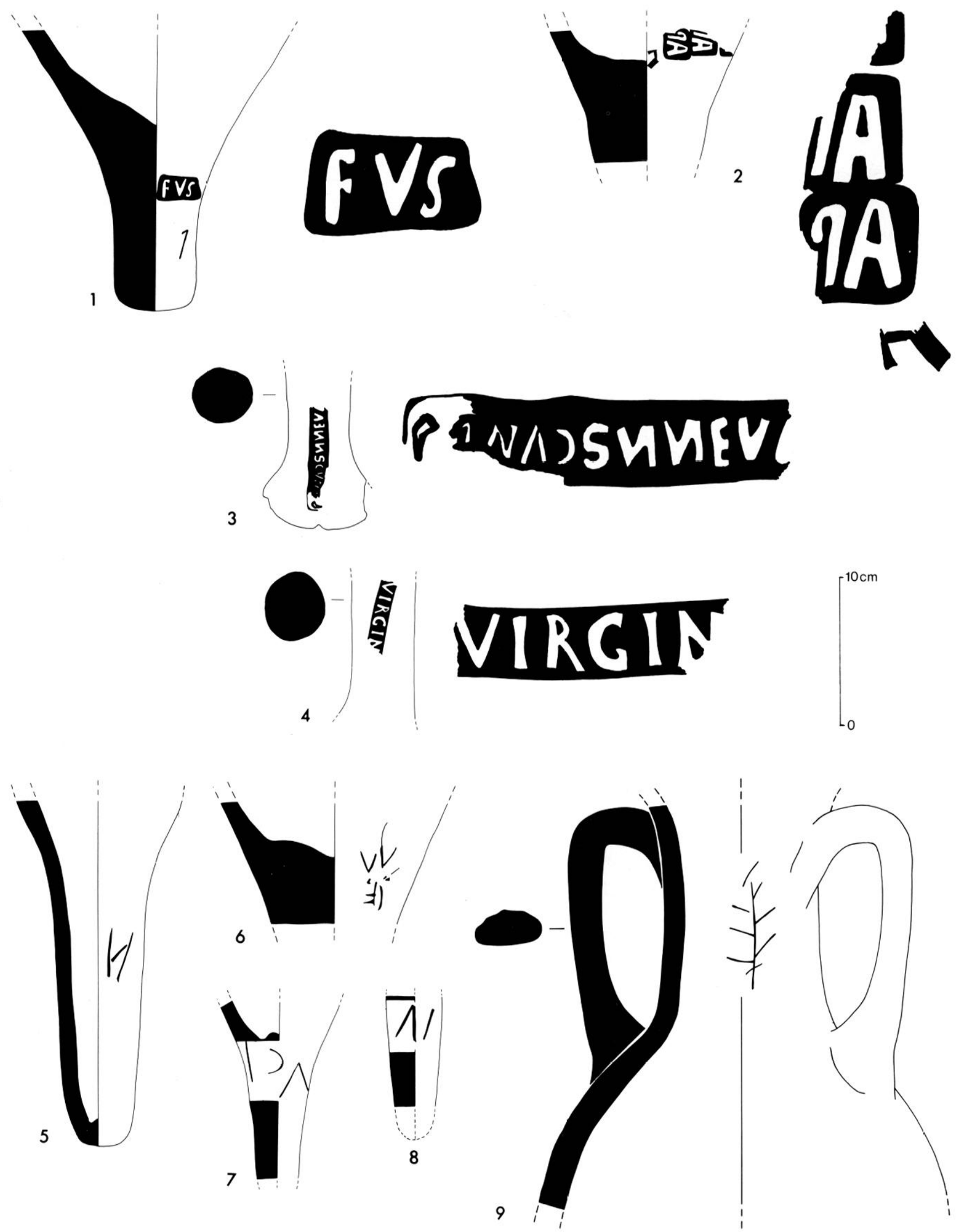

Fig. 33 - État III : marques et graffites sur amphores. 
Le foyer C ètait tapissé de grosses pierres et rempli de charbons de bois et de pierres plus petites. Il contenait assez peu de matériel : scories de fer. verre, céramique et ossements animaux.

L'ensemble du matériel retrouvé soit en couche, soit dans les fosses ou le foyer, était composé à nouveau d'un pourcentage élevé d'amphores (Dr. 20 et $2 / 4$, Pascual 1 et Tarraconaise, $58 \%$ ), de céramique culinaire et modelée $(11 \%)$, puis de céramique commune claire $(7,2 \%)$, de la sigillée de La Graufesenque $(6,4 \%)$, de céramique grise de Vaison $(5,6 \%)$. La datation qui s'en dégage correspond à celle de la couche de remblai (couche 8), c'est-à-dire la première moitié du II ${ }^{\mathbf{e}} \mathbf{s}$.

\section{Réoccupation de l'espace P7}

De la couche 3 recouvrant le sol de l'état. II à la couche 2 , première couche rencontrée en fouille contenant du matériel antique, on retrouve dans l'espace $\mathrm{P7}$ le même type de réoccupation que celui constaté en P5́.

La surface 3 est une surface sableuse recouverte de taches de cendre et de plaques de terre brune ou caillouteuse et percée par une fosse (fosse 3A) dans l'angle nord-est du secteur fouillé. Un fond d'amphore de Bétique reposait également sur la surface 3 (fig. 24).

Le matériel de la couche 3 et de la fosse $3 \mathrm{~A}$ contient, comme dans les couches correspondantes de la pièce 5 , une majorité d'amphores $(56,7 \%)$, de la céramique commune $(7,6 \%)$, de la céramique culinaire et modelée $(7 \%)$, un lot assez homogène de sigillée claire $\mathrm{A}$ et de céramique culinaire africaine $(5,4 \%)$. Les formes représentées dans cette catégorie (Hayes 9A, 14A, 16.1, 21, 23 et 196) nous permettent d'avancer une datation du milieu du II $^{\mathrm{e}} \mathrm{s}$. (confortée par un fragment de sigillée Drag. 18/31).

Dans la couche 2, un épais remblai de sable, on retrouve 79,6\% d'amphores (Bétique, Tarraconaise, Dr. 38 et $2 / 4, G 8, G 5, G 4)$. Le reste se répartit entre les autres catégories $(6,4 \%$ de céramique commune claire), avec une petite quantité de sigillée claire A et de culinaire africaine $(0,2 \%)$ dont les formes (Hayes $9 \mathrm{~B}$ et 197) nous fournissent des éléments de datation de la seconde moitié du $\mathrm{II}^{\mathrm{e}} \mathrm{s}$. (on trouve encore des fragments de Drag. 18/31 et 33), époque de l'abandon de l'état IIIA dans ce secteur.

\section{La pièce 3}

D'autres traces de réoccupation correspondant à l'état II sont visibles dans la rotonde (P3) où les couches 8 à 6 forment des recharges et des remblais de rattrapage de la pente déjà existante aux états précédents (fig. 8).

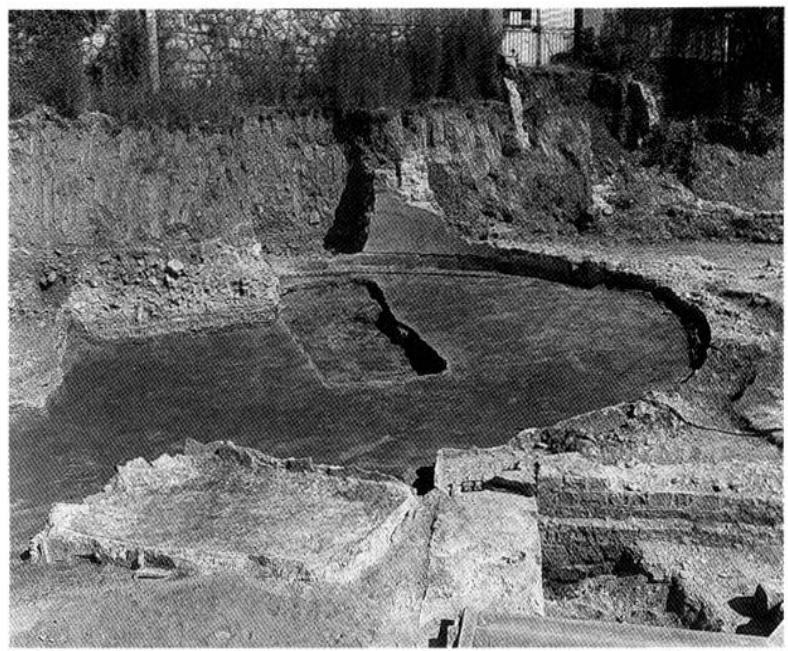

Fig. 34 - La cuve M38 obturant partiellement l'entrée de la pièce 3.

- La couche 8 contient du sable assez grossier mêlé par endroits à des lentilles d'argile.

- La couche 7 est constituée de petits gravats (mélange d'enduits peints et de mortier), avec une recharge de sable fin à l'ouest (couche 7a) et une couche d'argile au sud (couche $7 \mathrm{~b}$ ).

- La couche 6 est un remblai de terre marron. C'est à partir de la surface 5, argileuse, homogène et très damée, qu'a été creusée la cuve à chaux (M38) à l'entrée de la rotonde, qu'elle obture partiellement (fig. 31). Il s'agit d'une structure de plan trapézoïdal $(2,80 \times 2,10 \mathrm{~m}$ à l'ouest et $1,60 \mathrm{~m}$ à l'est $)$, en béton de tuileau; l'intérieur, légèrement concave, forme une cuvette de $0,20 \mathrm{~m}$ de profondeur conservée. Une bordure faite de tuiles plates posées de chant subsistait dans le béton. Le comblement de M38 est composé de cinq couches (gravillons provenant de la décomposition du béton de construction, argile verte, blocs quadrangulaires d'argile rubéfiée semblant provenir d'une sole de four, couche d'argile rubéfiée, terre meuble recouvrant le tout). Le matériel était composé essentiellement de tessons d'amphores (55 à $100 \%$ selon les couches).

Les éléments de datation sont très rares : un tesson de céramique culinaire africaine de type Hayes 23 dans la couche 8 ; trois fragments identifiables de La Graufesenque ne dépassant pas le début du II $^{\mathrm{r}} \mathrm{s}$. dans la couche 7 ; trois fragments identifiables de datation similaire dans la couche $\overline{5}$. Le comblement $\mathrm{du}$ bassin fournit deux fragments de sigillée claire $\mathrm{B}$ et un de sigillée claire $A$. Nous proposons donc pour l'ensemble de ces couches une datation de la première moitié du $\mathrm{II}^{\mathrm{e}} \mathbf{s}$. 


\section{LA CÉRAMIQUE COMMUNE} DE L'ÉTAT IIIA

Fig. 35 et tabl. III, p. 218.

Elle provient essentiellement de la pièce 5 couche 8 et de la pièce 7 couches 1 et 2 : en dehors des amphores, c'est la catégorie la plus importante; elle se divise, encore une fois, en céramique à pâte claire locale (la majorité) dont les caractéristiques de pâte ont déjà èté définies et en céramique importée. Contrairement à l'état précédent, ce sont les formes fermées qui dominent.

\section{Fabrications locales}

\section{Formes ouvertes}

Fig. $35, n^{0 \times} 1$ et 2.

1 coupe à anses en boudin rapportées, avec un pou-
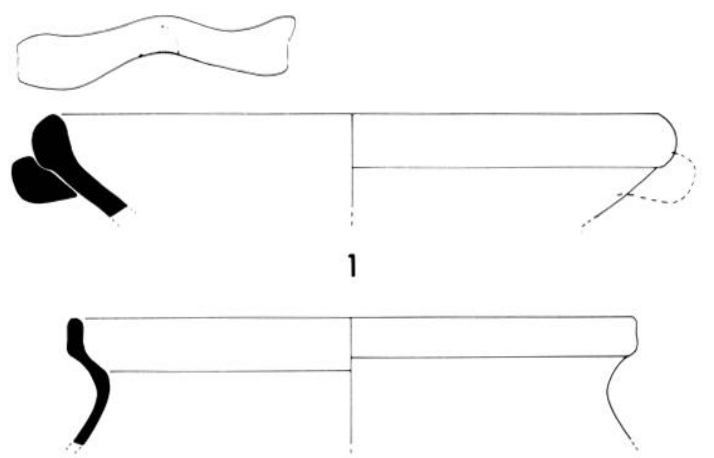

3

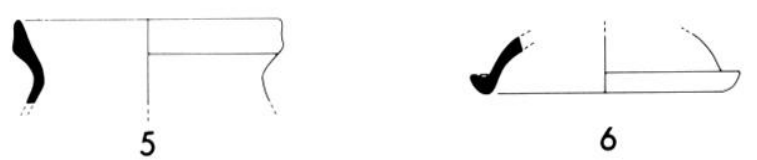

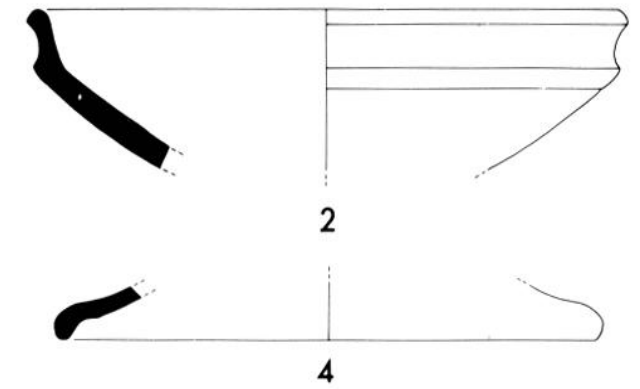
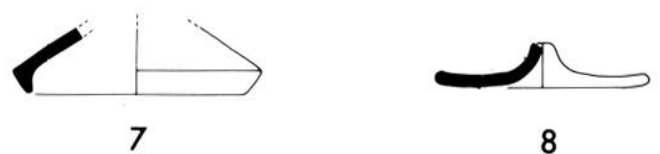

8

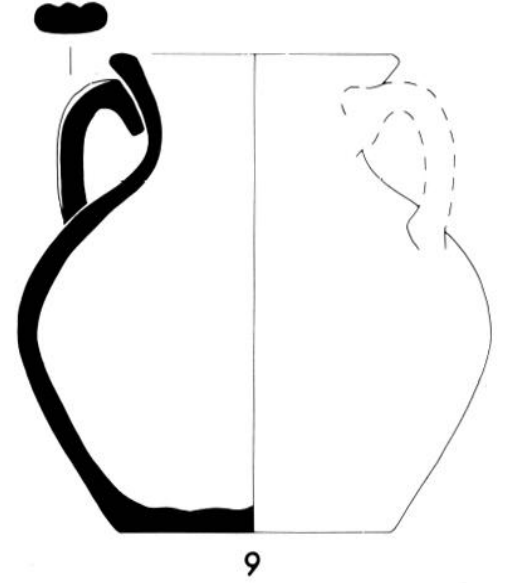

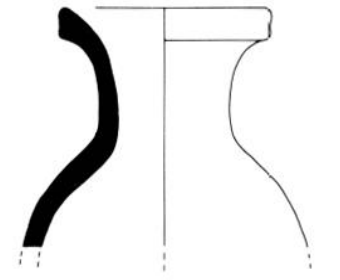

10

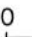

$10 \mathrm{~cm}$

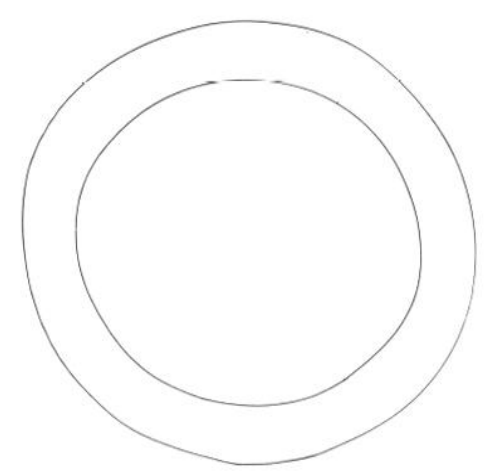

)

12

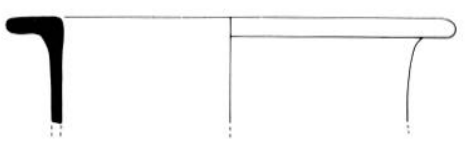

13

11

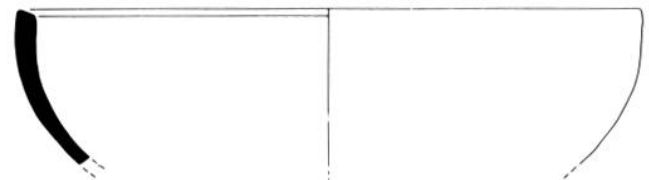

Fig. 35 - La céramique commune de l'ètat III. 
(ier central (sorte de "tian" provençal), à lève en amande, et panse très évasée.

2 coupe tronconique, peut-être munie d'un bec verseur: bord concave, carène marquée (Rivet, à paraître, type $17 \mathrm{a} ; 1980$, type $39 \mathrm{~b}$; Pasqualini, 1988. type $\overline{)})$.

\section{Formes fermées}

Fig. $35, n^{\circ} 3$ à 10.

3 urne à bord caréné, similaire à de nombreux exemplaires présents dans la nécropole du Pauvadou/ I agon Bleu (tombes $39 n^{\circ} 4 ; 42 n^{\circ} 1 ; 43 n^{\circ} 1 ; 47$ $\mathrm{n}^{\circ} 2 ; 48 \mathrm{n}^{\circ} 1$, pour ne citer que quelques exemples ; inédites).

4 couvercle à bord rentrant, ayant peut-être appartenu à l'urne n" 3 (Bèrato et alii, 1986, type 8).

5 urne à lèvre triangulaire, de diamètre inférieur à l'urne no 3 (Pasqualini, 1988, B1/B2).

6 coupelle ayant servi de couvercle, à lèvre pendante (Rivet, à paraître, type 20 ; 1980, type '2a).

7 coupelle ou couvercle, à bord rentrant caréné (Rivet, 1980, n०1).

8 couvercle à préhension pincée et trouée, bords relevés.

9 vase à deux anses, lèvre aplatie, fond plat, panse ovoïde. Les parallèles ne sont pas entièrement satisfaisants, mais citons pour mémoire: Santamaria, 1985, fig. $6 \mathrm{n}^{\circ} 3$; Dyson, 1976, V.I)., C-ware, jars 82, 83, 85; Heraclea Caccabaria, 1988, no 166, aux dimensions similaires et de fabrication sans doute fréjusienne.
10 vase fermé à lèvre en listel et col biconcave, sans anses; peut-être d'origine locale (comparaisons lointaines avec Rivet, 1980, n' 28; T)yson, 1976, PD168, I)-ware).

\section{Céramique importée}

Fig. 35, $\mathrm{n}^{\mathrm{os}} 11$ à 13.

11, 12 pots ovoïdes en céramique commune sombre de la vallée du Rhône (Desbat et alii, 1979).

13 bassine en céramique brune italique, légèrement ovale (Dyson, 1976, Late Shops, K-Ware, L.S5்).

\section{ÉTAT IIIB : LE REMBLAIEMENT}

Dans cette rotonde, on note ensuite un phénomène qui affecte la moitié ouest du site et une partie du bâtiment de l'état II (P7, couche 2) : il s'agit du remblaiement massif de cette zone par des gravats issus probablement du démantèlement d'une autre construction située soit au nord, soit à l'ouest du terrain fouillé. Fn effet, la couche 8 de ce remblai est composée de morceaux de maçonnerie, de plàtre et de fragments de voûtains de plafonds, de pierres de claveaux de luf, de fragments de sols de mosaïque blanche avec leur nucleus et leur rudus d'origine, de morceaux d'enduits peints, de plaques de marbre provenant d'un opus sectile. Quelques éléments d'architecture y ont été retrouvés : un seuil en grès; une base de colonne fragmentaire moulurée (diamètre minimal : $45 \mathrm{~cm}$ ) et un autre fragment (diamètre minimal : $40 \mathrm{~cm}$ ) tous deux en marbre blanc; deux chapiteaux de pilastre (longueur : $102 \mathrm{~cm}$; hauteur : $26,5 \mathrm{~cm}$ ) également en marbre blanc (fig. 36 ) et une colonne géminée en grès blanc.
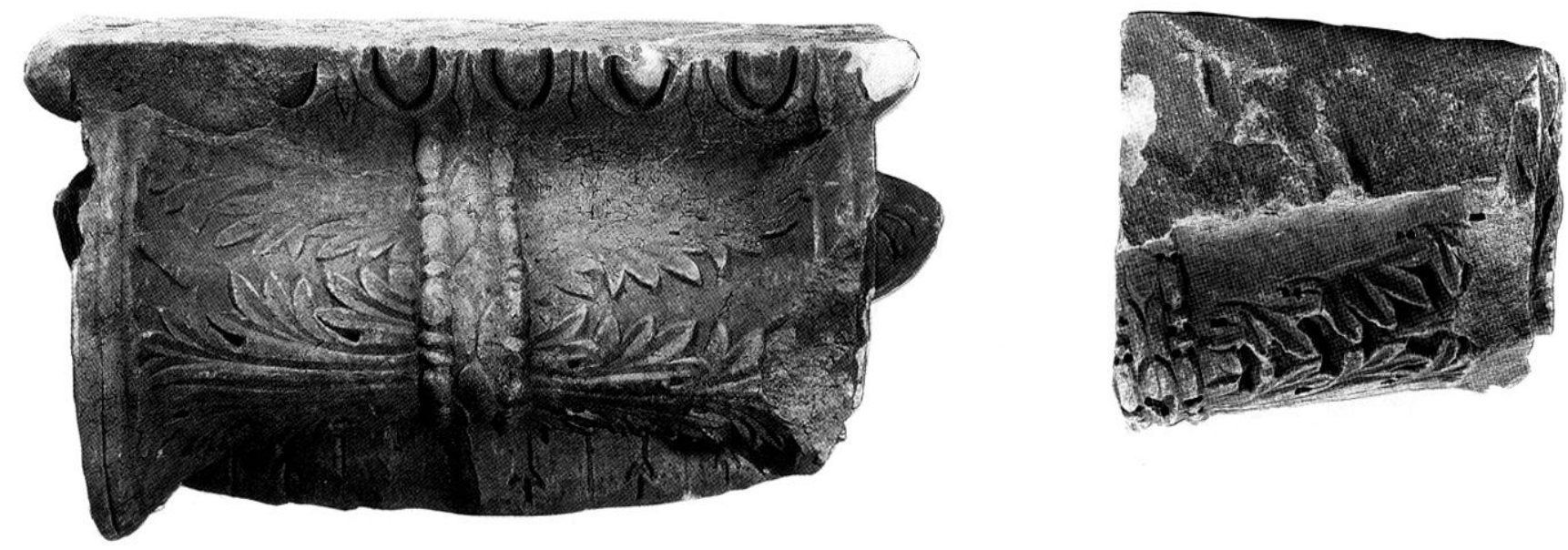

Fig. 36 - Chapiteaux en marbre trouvés dans la couche 8 . 
Cette couche, d'une épaisseur de $1,43 \mathrm{~m}$ à l'ouest, se réduit vers l'est à $1,13 \mathrm{~m}$ au niveau de la rotonde, pour se terminer en pente douce vers le mur M9 de l'état IA ; ce remblai de gravats vient buter contre le parement interne du mur M11 en P3, qui alors lui sert de soutènement. A partir du mur M9, un remblai beaucoup plus sableux conlenant des fragments de tuiles, de briquettes, d'enduit blanc et d'amphores, vient recouvrir le sol d'argile correspondant à l'état II et rejoint la couche 2 de la pièce 7 .

Très peu de matériel a été recueilli dans cette couche 8 , car elle a été décaissée à la pelle mécanique pour les raisons de délais évoqués plus haut. Hormis les éléments de revêtement ou de décoration déjà mentionnés, il a été trouvé une firmalampe (type Bucchi IXC) marquée STROBILIS. La majeure partie du matériel est composée d'amphores (Tarraconaise, Gauloise, Bétique : $67,4 \%$ ) et de céramique commune à pâte claire $(24,4 \%)$.

Toute une partie du site est donc fortement surélevée et cette modification profonde du paysage restera marquée jusqu'en 1986, puisqu'un mur de soutènement moderne orienté nord-sud soulignait la différence de niveau entre la partie ouest et la partie est du site, reprenant l'alignement antique des murs M9 et M11.

Fondée directement dans cette couche de gravats, un égout (drain 21) traverse la partie ouest du site, en empruntant une direction sud-ouest/nord-est (fig. 37). Il s'agit d'une construction en petit appareil régulier de grès (moellons de $20 \mathrm{~cm}$ d'épaisseur),

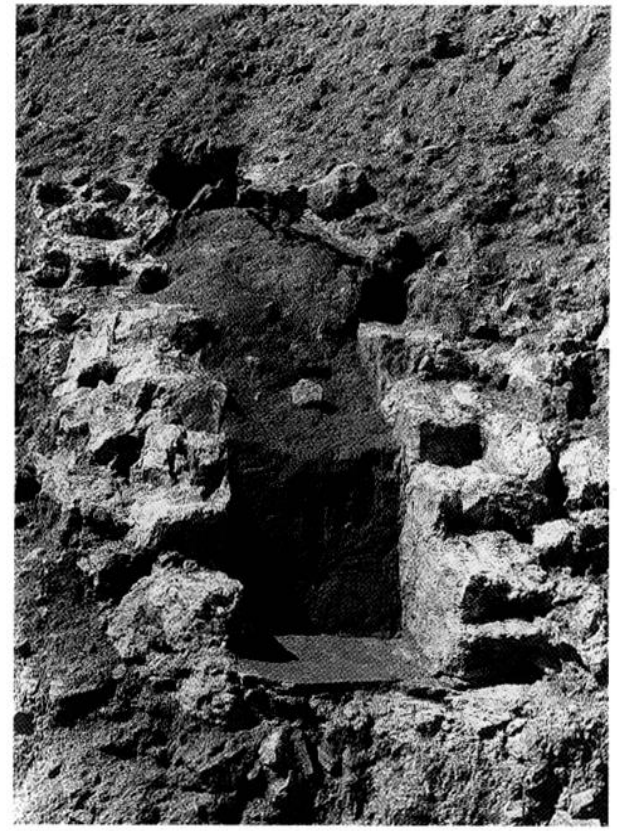

Fig. 37 - Le drain 21 en coupe. parementée à l'intérieur et noyée à l'extérieur dans de la maçonnerie. Le muret sud est bâti sur un lit de mortier; le fond est fait de dalles de terre cuite d'environ $40 \mathrm{~cm}$ de large et $47 \mathrm{~cm}$ de long. Le muret nord est construit directement sur le rebord de ces dalles. La couverture est composée de tegulae posées en bâtière sur le sommet des murets maçonnès et un regard de calcaire en place, de 55 sur $60 \mathrm{~cm}$ de côté a été trouvé. La hauteur de cette canalisation était de $1,30 \mathrm{~m}$ et sa pente de $13 \mathrm{~cm}$ pour une vingtaine de mètres, vers le nord-est.

Cet égout était entièrement comblé par deux couches de terre brune assez pure et fine qui contenait, à l'emplacement fouillé, très peu de matériel réparti sur les catégories suivantes: amphores (33\%); céramique commune claire, grise culinaire et engobée, locales (11\% de chaque catégorie); lampes $(5 \%)$; sigillée sud-gauloise $(16 \%)$; sigillée claire A $(11 \%)$.

Dans la tranchée de fondation du drain, à l'emplacement fouillé, il y avait un peu plus de matériel (fig. 35, nos 14 et 15) : amphores (Gauloise, Dr. 2/4, $38: 44 \%$ ); céramique commune claire, culinaire modelée, culinaire brune italique $(2,5 \%$ de chaque catégorie); céramique grise (5\%); céramique grise de Vaison $(5 \%)$; sigillee sud-gauloise $(23,5 \%$, dont 3 panses décorées); culinaire africaine Hayes $23(5 \%)$.

A partir de ces quelques données et en les mettant en relation avec la couche 8 et la stratigraphie générale de ce secteur, nous proposons une datation du $\mathrm{II}^{\circ} \mathrm{s}$. pour le remblai et la canalisation 21 , et plus probablement, de la seconde moitié du $\mathrm{II}^{\mathrm{e}} \mathrm{s}$., si l'on prend en compte les données chronologiques fournies par la couche 2 de l'espace P7.

\section{INTERPRÉTATION}

L'état III se caractérise donc par une réoccupation du monument public et de la rotonde vers la première moitié du $\mathrm{II}^{\mathrm{e}} \mathrm{s}$. (état IIIA), puis par un exhaussement global du site à l'ouest durant la seconde moitié du II $^{\mathrm{e}} \mathrm{s}$. (état IIIB).

La nature de la réoccupation IIIA est assez simple à concevoir : un bâtiment déserté el facilement accessible, ouvert, est réutilisé partiellement comme abri temporaire, peut-être même comme lieu de stockage de provisions (dolium et amphores de la fosse 3 en P5). En même temps, une activité de récupération des revêtements de marbre ou de calcaire est établie sur l'ancienne esplanade : la cuve M38 et la surface 5 dans la rotonde.

Assez rapidement, pour des raisons qui nous échappent, on éprouve le besoin de surélever de manière importante le niveau de l'esplanade et, 


\section{Fig. 38 -}

Antéfixe trouvé

dans la couche 8 .

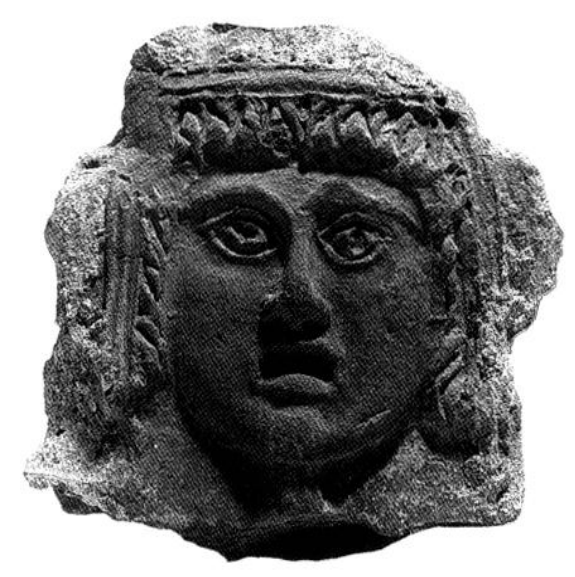

cause ou effet, il sera impossible de le dire, les gravats provenant d'un bâtiment proche sont amenés dans ce but. Il est cependant possible d'avancer une explication à cet énorme apport de matériaux propres, aérés et secs que constituaient les gravats de la couche 8 : l'humidité ambiante. Ainsi, à l'instar des vides sanitaires d'amphores enfouis dans le sable de comblement des états précédents, cette vaste plate-forme dont nous ne connaissons que la limite est, a été peut-être une solution technique plus facile à mettre en cuvre et plus efficace pour résoudre les problèmes de remontée d'eau et d'humidité que ce secteur semble toujours avoir connu.

Le bâtiment détruit devait être relativement luxueux, si l'on en juge par les fragments de décoration qui nous sont parvenus. D'autres couches et fosses plus tardives ont révélé des éléments de récupération de mème nature : une lète el une portion de statuette, un chapiteau de pilastre et un demi chapiteau de style ionique en marbre ${ }^{18}$ (fig. 36) et deux antéfixes (largeur : $18 \mathrm{~cm}$, hauteur : $15 \mathrm{~cm}$ ) en terre cuite (fig. 38).

Il ne pouvait s'agir de la récupération des éléments de revêtement du monument de l'état II, car ceux-ci avaient déjà dû être au moins partiellement arrachés durant l'état IIIA et, de plus, les murs étaient encore debout jusqu'au $\mathrm{vi}^{\mathrm{e}} \mathrm{s}$. au moins (voir infra, l'état IV, p. 201).

L.e niveau d'utilisation de cette couche est à mettre en rapport avec l'égout 21, mais l'installation postérieure des silos de l'état $V$ semble avoir détruit les surfaces liées à ce remblai.

De fait, au $\mathrm{II}^{\mathrm{e}} \mathrm{s}$, , on devait apercevoir à partir du bassin portuaire un bâtiment somme toute peu changé extérieurement et, à l'ouest et au nord, une terrasse venant mourir en pente douce contre cette construction.

18 Datés de l'époque julio-claudienne par A. Roth Congès, que nous remercions ici.

\section{ÉTAT IV}

Durant l'Antiquité tardive, l'activité se concentre exclusivement dans l'édifice public, encore conservé en élévation. Les pièces 4 et $\overline{5}$ deviennent des espaces habités, tandis que la pièce 10 est transformée en un vaste dépotoir. Ailleurs, notamment dans la pièce 6 et le portique 11 , les traces sont plus ténues et ponctuelles (fig. 39).

\section{ÉTAT IVA}

Dans la pièce 4 , le sol antique continue d'être utilisé de sorte que les seuls vestiges de la réoccupation se limitent aux nouveaux aménagements installés dans le béton de tuileau (fig. 40).

La salle est traversée du nord au sud par deux tranchées (Tr. 29 et $\mathrm{Tr} .30$ ) distantes de $3,50 \mathrm{~m}$ et. creusées respectivement à $1,20 \mathrm{~m}$ et $0,90 \mathrm{~m}$ des murs 13 et 25 . Elles constituent deux saignées d'une largeur moyenne de $0,70 \mathrm{~m}$ pour une profondeur de $0,60 \mathrm{~m}$ (fig. 41 ). La tranchée 30 ne portait aucune trace d'un éventuel revêtement, en revanche le fond de la tranchée 29 était partiellement recouvert de mortier, d'argile ou, pour la partie sud, de fragments de tuiles posés à plat qui formaient un radier (fig. 42). Les deux cavités, dans la mesure où leur vocation devait être identique, avaient pu bénéficier d'un tel dispositif certainement destiné à servir d'assise à des structures, probablement des murs. Malheureusement la fouille n'a révélé aucun témoin de ces constructions dont la disparition totale peut s'expliquer par une récupération exhaustive ou par l'emploi de matériaux périssables.

Il nous faut donc restituer dans cette pièce deux murs parallèles qui définissent un nouvel espace d'une superficie de $24 \mathrm{~m}^{2}$. Nous ne pensons pas que ces nouveaux parements avaient été édifiés dans le seul but de modifier l'espace interne mais qu'ils répondaient à un besoin technique précis. Ainsi ils ont pu remplacer les murs du bâtiment alors en mauvais état ou soutenir une toiture déficiente. Après la récupération des matériaux qui les constituaient les tranchées ont servi de dépotoir et se sont comblées d'une terre noire associée à des fragments de céramiques, de coquillages, des charbons de bois et des cendres.

Le béton a également été entaillé pour permettre l'installation d'un foyer (foyer A) (fig. 43) contre le nouveau parement ouest de cette pièce. Il s'agit d'une structure fruste, de forme ovoïde, mesurant $1,80 \mathrm{~m}$ sur $1,60 \mathrm{~m}$, dont la surface d'utilisation est formée simplement de quelques fragments de tuiles et de pierres accumulés essentiellement à l'est. 


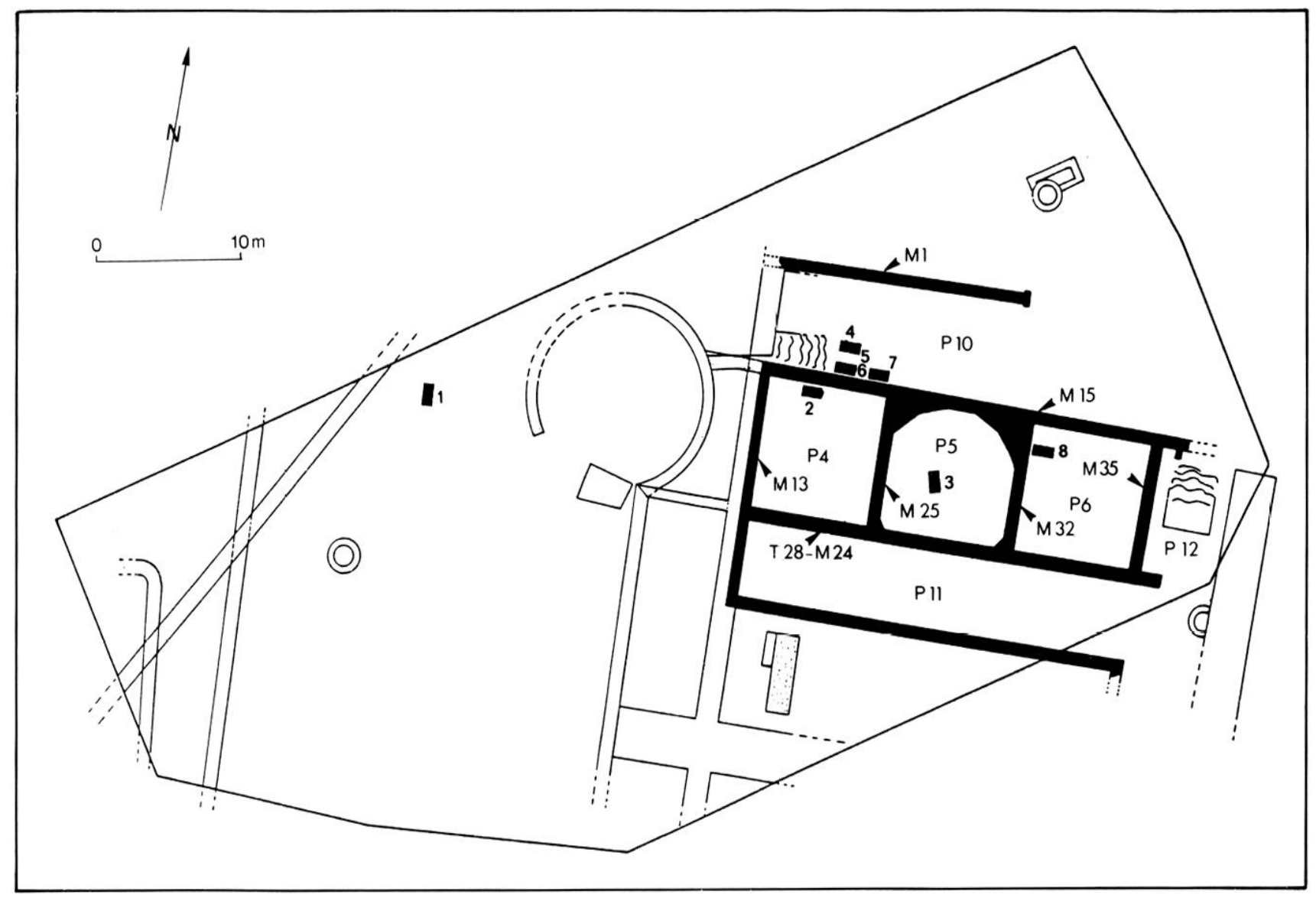

Fig. 39 -. Plan sélectif de l'état IV.

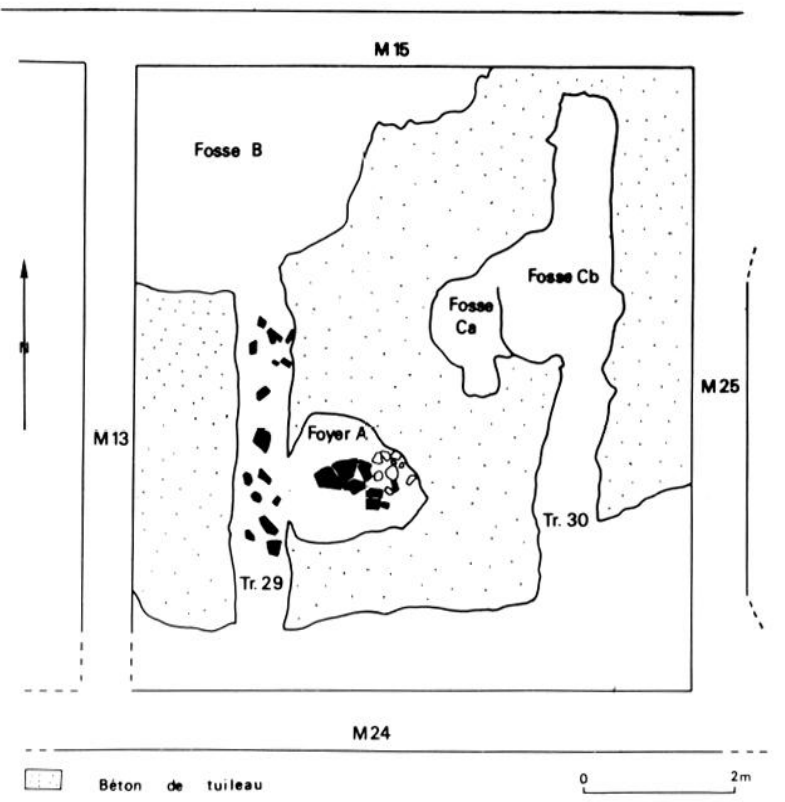

Fig. 40 - Pièce P4. Plan des installations tardives.
Des tessons de céramique et des coquillages ont été trouvés au contact des tuiles et des pierres; ils n'avaient pas subi l'action du feu et avaient dû être jetés là peu de temps après l'abandon du foyer.

\section{Éléments de datation}

Tabl. IV, p. 219.

Les céramiques contenues dans le foyer sont des sigillées claires I) de la première génération (forme Hayes 59, 61 et 91) (CATHMA, 1984, p. 34), qui permettent de situer son abandon et peut-être celui de la pièce vers le milieu du $v^{\prime \prime} s$. Cette céramique fine est associée à un bord d'amphore du type Keay LXII Q (deuxième quart du v's.-milieu du vi"s.) (fig. 44, no 1) (Keay, 1984, p. 309-350) et une anse de la forme Late Roman Amphora 1 (Peacock, Williams, 1986, p. 185).

I)ans l'espace P10, la fouille a révélé la présence d'un vaste dépotoir qui n'a pu ètre reconnu de manière exhaustive mais pour lequel nous avons 


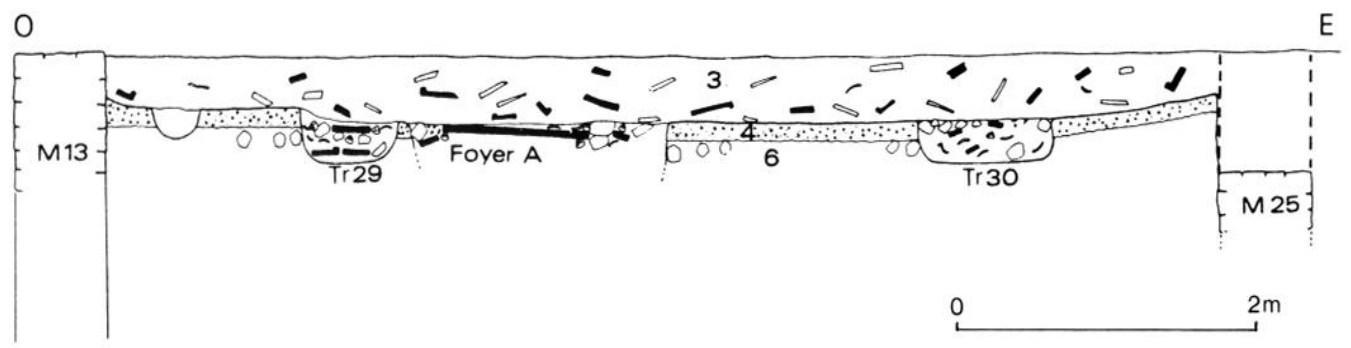

Fig. 41 - Pièce P4 : coupe stratigraphique ouest/est.

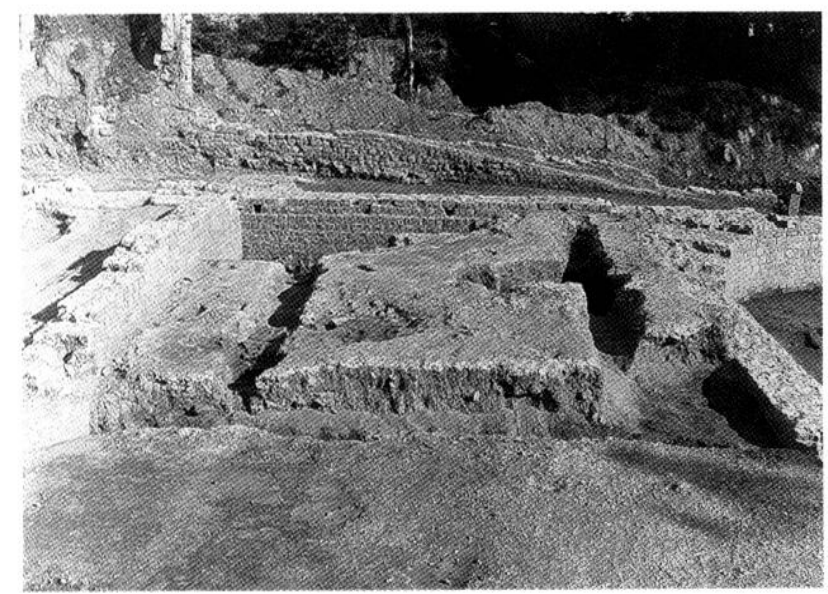

Fig. 42 - Vue de la pièce P4. négatifs de cloisons tardives.

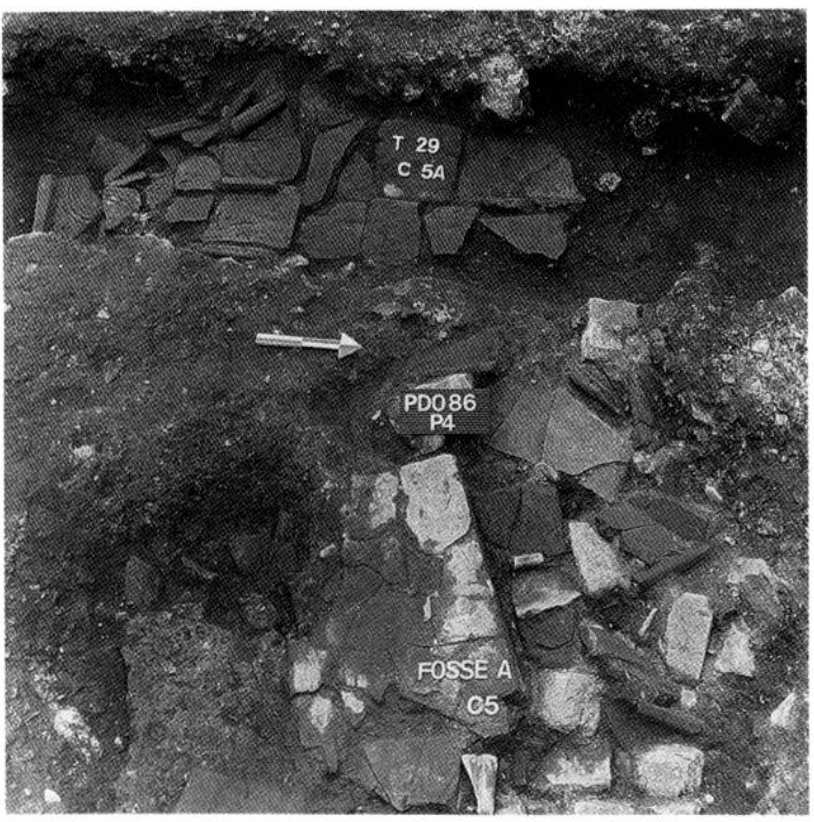

Fig. 43 - Pièce P4 : vue du fover $A$.

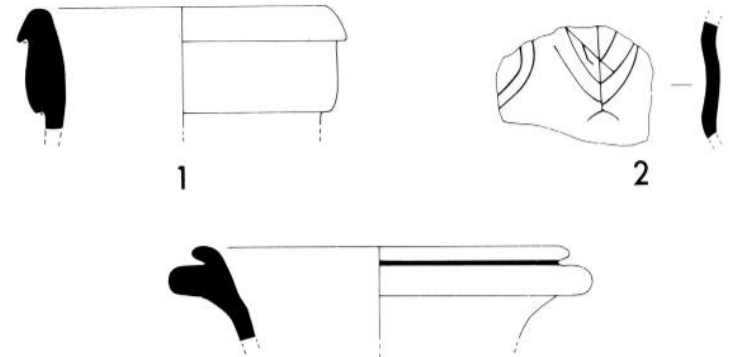

3
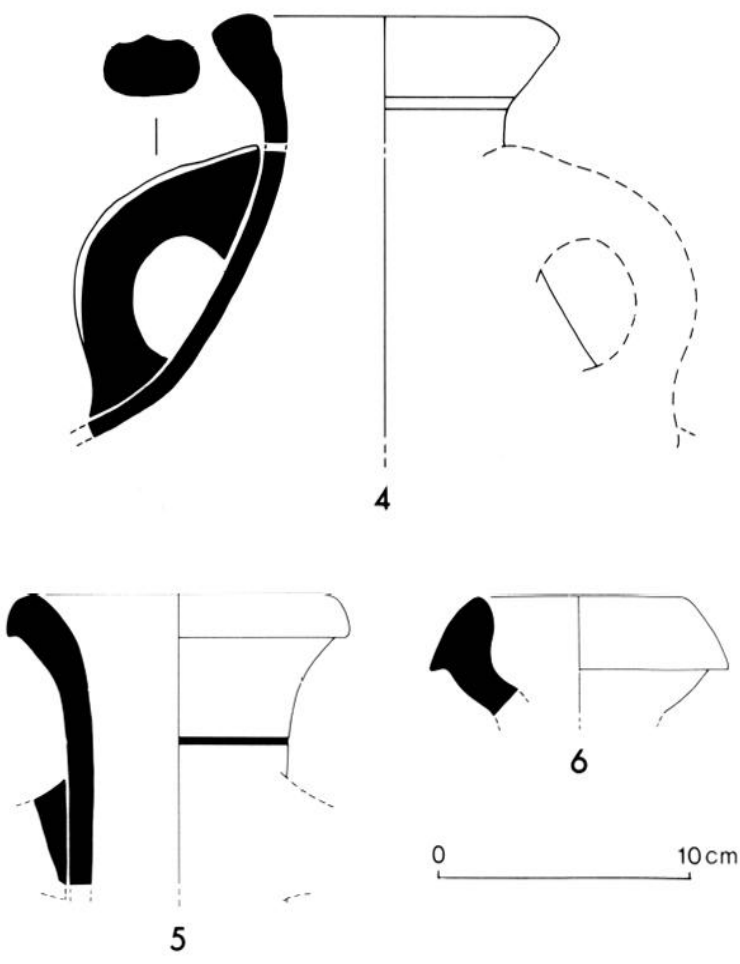

Fig. $44-$ Amphores. 


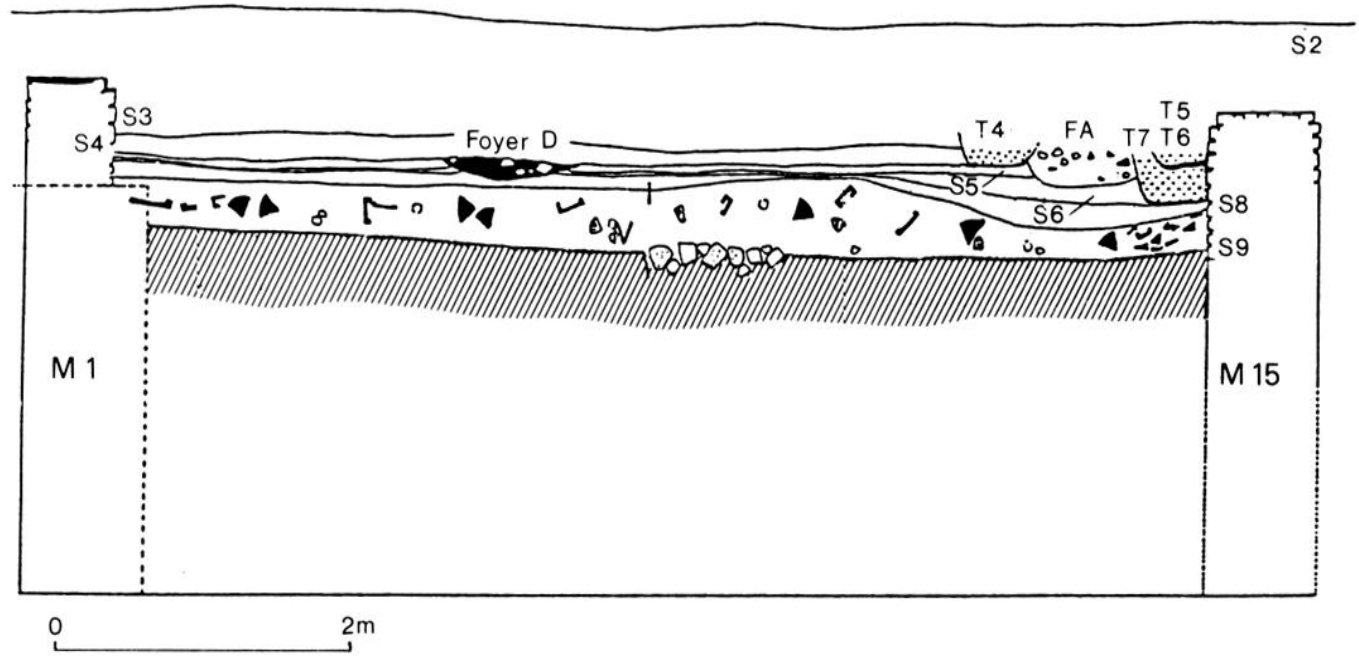

Fig. 45 - Dépotoir de la pièce P10 : coupe stratigraphique nord/sud.

individualisé trois niveaux successifs (couches \$8, sy et S10) (fig. 45). Ces trois couches présentaient le même aspect: terre marron, contenant des tuiles, des tubulures, des quarts de rond ainsi que d'autres matériaux de construction, mais surtout un important matériel de l'Antiquité tardive ${ }^{19}$. L'étude des sigillées claires D permet de le placer dans la première moitié du $v^{\prime \prime} s$. Parmi les amphores tardives présentes citons les formes LXIIA et $Q$ de Keay, une anse du type Late Roman Amphora 3 (début du IV s. - fin du vie s.) (Leguilloux, 1988, p. 32) ainsi que deux fragments de panse du même type où figure un graffite représentant un chandelier à sept branches (fig. 44, no 2), un bord du type Maña C (fin du $111^{\mathrm{e}}$ s. - début du ve s.), (fig. 44, $\mathrm{n}^{\mathrm{o}} 3$ ) (Peacock, Williams, 1986, p. 151-152), un bord du type Africaine II en pâte bordeaux contenant de nombreuses inclusions blanches et noires (fin du $\mathrm{II}^{\mathrm{e}} \mathrm{s} .-\mathrm{IV}^{\mathrm{e}} \mathrm{s}$ ) (fig. 44, no 4) (Peacock, Williams, 1986, p. 155-156), un bord du type Ostia IV (IV - ve s.) (fig. 44, no 5 ) (Peacock, Williams, 1986, p. 153-154).

Dans le portique (P11), les témoins de la réoccupation se limitent à trois excavations creusées dans le béton, il s'agit des fosses $\mathrm{D}$ et 3a ainsi que d'un foyer $3 \mathrm{~b}$, de forme circulaire et comblé de cendres, de charbons de bois et d'un fragment d'inscription en marbre dont les lettres sont très mutilées.

19 La masse globale du matériel issu de ce dépotoir (soit 3445 fragments) correspond à plus de la moitié de l'ensemble des tessons étudiès pour celte période.

\section{$E_{T A T} I V B$}

Dans la pièce P5, le premier niveau de l'Antiquité tardive, couche 6 , est constitué d'une fine épaisseur de terre mêlée de mortier, surface $6 c$, recouverte de deux recharges faites d'argile dans la partie médiane (surface 6a) et de sable au sud (surface 6 b) (fig. 30).

Ce niveau avait été percé de deux fosses (F1 et F2) (fig. 46) comblées respectivement de terre noire contenant peu de matériel et de gravats de construction (pierres, fragments de tuiles et d'enduits peints rouges) associés à quelques tessons de céramiques. Nous n'avons par ailleurs décelé aucune trace d'occupation importante, seule une poche de cendres de forme circulaire située à l'angle sud-ouest de la pièce peut être signalée, nous l'avons appelée foyer B mais elle est en réalité le résultat d'une activité ponctuelle.

Pour cette période, la couche 5 est le seul niveau d'occupation de cette pièce, il s'agit d'une épaisse couche de terre jaune, sableuse ou argileuse, contenant un matériel céramique relativement important (723 tessons). Elle a connu une phase d'occupation plus longue et a bénéficié d'aménagements plus conséquents (fig. 46).

Un foyer (foyer A) a été retrouvé dans l'angle nord-ouest de la pièce. Il avait pris place dans une fosse quadrangulaire, bordée de pierres dont cer- 
taines avaient éclaté à la chaleur. Cette installation rudimentaire était remplie de bois calciné et de cendres sur lesquels des moellons avaient été disposés après la dernière utilisation.

Un bloc de grès quadrangulaire, de $0,60 \mathrm{~m}$ sur $0,80 \mathrm{~m}$ pour une hauteur de $0,70 \mathrm{~m}$, avait été inséré profondément dans le sol (fig. 47) ct constituait un socle particulièrement solide. Cet élément avait probablement èté arraché à un édifice antique; il présentait une moulure et un fragment de béton de tuileau était encore accroché contre sa face inférieure. Sa surface était creusée en son centre d'une cavité carrée de $25 \mathrm{~cm}$ de côté qui a pu, par exemple, recevoir un pilier destiné à soutenir la toiture, ce qui explique sa position centrale.

Au sud-est de ce bloc, nous avons retrouvé un muret (M31) peu fondé, construit en pierres sèches, long de $1,90 \mathrm{~m}$ pour une largeur de $0,60 \mathrm{~m}$ et conservé sur une hauteur de $0,30 \mathrm{~m}$. Il est difficile de préciser sa fonction, car nous ne connaissons pas son aspect initial, peut-être s'agissait-il d'un simple aménagement domestique.

\section{Éléments de datation}

Les rares tessons recueillis dans la couche 6 ne fournissent pas d'éléments de datation précis et, à l'exception de deux fragments informes de DSP, l'ensemble du matériel était composé de céramiques communes. Cependant, nous avons pu constater que le matériel apparaît dans les mêmes proportions que dans la couche 5 (fig. 48), ce qui nous permet peutètre d'exclure une discontinuité trop importante entre ces deux contextes.

La couche 5 contenait peu de céramique fine, toutefois la présence d'un tesson de sigillée claire D de la forme Hayes 99, associé à un matériel qui diffère, par les proportions respectives des différents types représentés, de ce que nous avons pu observer dans la pièce 4 (fig. 48), nous permet de placer ces niveaux dans le courant du vi ${ }^{e}$ s. ou peut-être à la fin du ve s. ${ }^{20}$.

20 La forme 99 apparait à Marseille à partir de la période $2 \mathrm{~A}$ phase 3 (fin du $v^{\prime \prime}$ s. - début du $v^{*}$ s.) et est particulièrement importante dans la phase 4 (courant du $\mathrm{vI}^{\mathrm{e}} \mathrm{s}$.) (Bonifay, 1983). La céramique grise prend davantage d'importance dans ces niveaux, or on constate que sur d'autres sites de Provence ou du Languedoc ce type de production est mieux représenté dans les contextes de la fin du $v^{\prime \prime} s$. et du vi"s. (C.A'TIMA. 1984, p. 37).
La salle $\overline{5}$ a donc été occupée après l'abandon de la salle mitoyenne $\mathrm{P} 4$ dont le béton est alors percé de deux fosses $\mathrm{Cb}$ et $\mathrm{Ca}$, creusées successivement contre la tranchée 30 (fig. 40). Ces deux cavités imbriquées ont servi de dépotoir; la première avait été comblée de sable auquel se mêlaient quelques os animaux et fragments de céramiques, tandis que la seronde contenait, associés à des pierres, des os, des tessons, ainsi que des cendres et des charbons de bois. Ce comblement est daté du $\mathrm{vi}^{e} \mathrm{~s}$. par deux bords de sigillée claire I) de la forme Hayes 88 (tabl. V, p. 220).

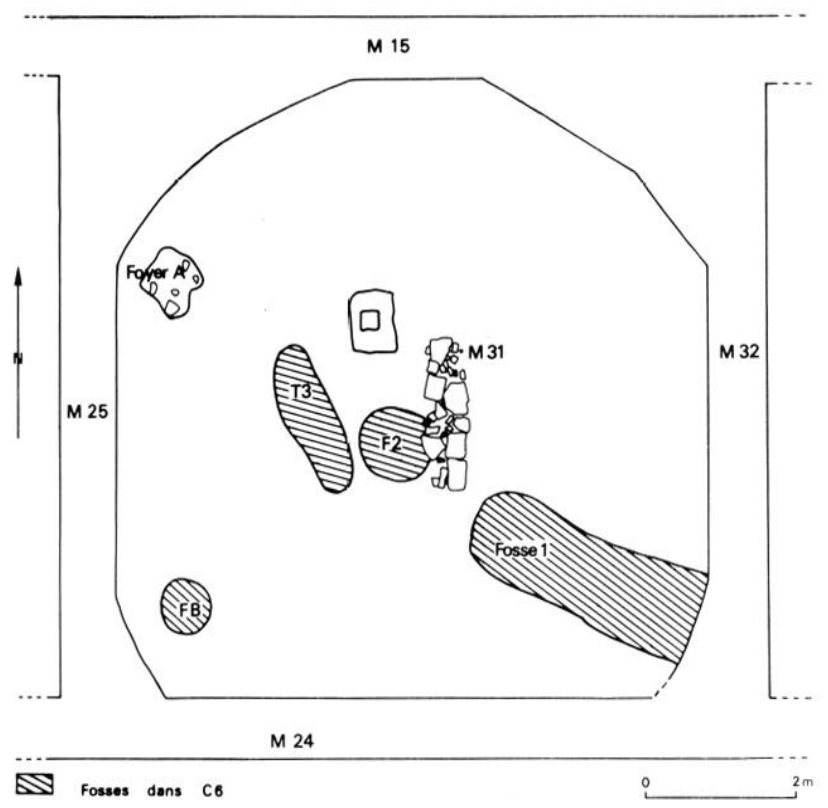

Fig. 46 - Pièce P5. Plan des installations tardives.

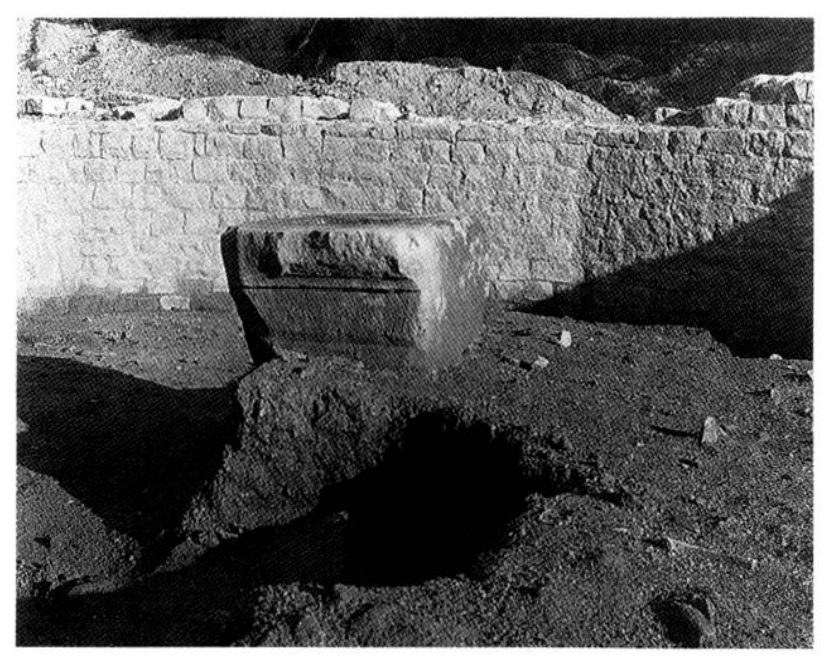

Fig. 47 - Support central en place dans la pièce 45 . 


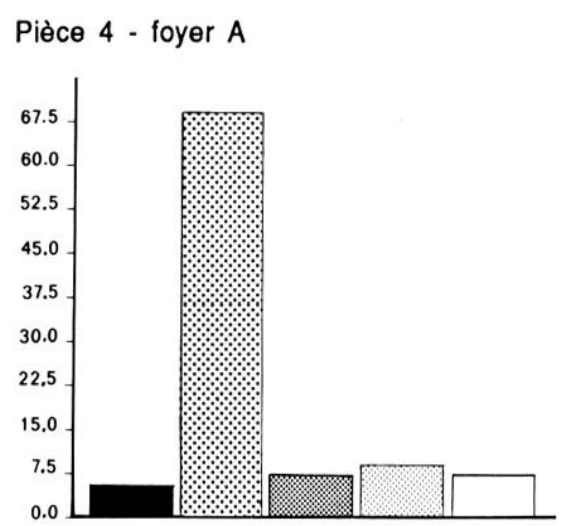

Pièce 10 - dépotoir

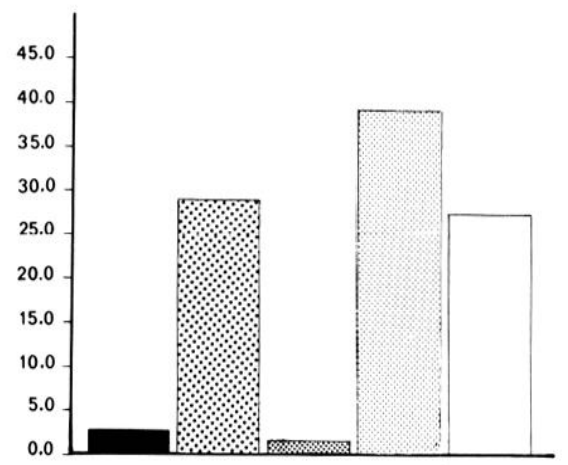

Pièce 5 - phase I

Pièce 5 - phase II
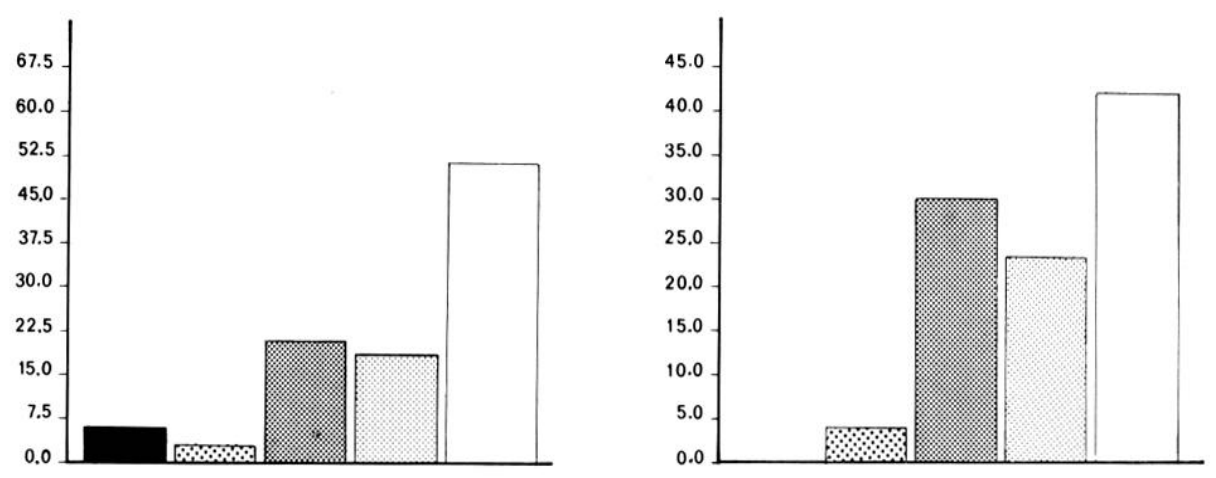

DSP

claire D

communes brunes

communes grises

communes claires

Fig. 48 - Diagrammes de comparaison.

\section{LES SÉPULTURES}

Sept tombes contemporaines des phases IVA et IVB de l'occupation du site ont été découvertes dans les pièces 5,6 et $10^{21}$ (fig. 39).

Dans la pièce $P 5$, l'inhumation 3 a été aménagée dans une fosse de $1,90 \mathrm{~m}$ sur $0,70 \mathrm{~m}$ creusée à partir de la surface 6 . Le squelette, orienté nord-sud, reposait directement sur le sol, tandis que les parois de la tombe étaient faites d'un parement de pierres de modules divers, assemblées sans liant, formant un coffrage. La présence de trois clous disposés le long du parement est pourrait indiquer l'utilisation concomitante d'une structure en bois.

21 L'étude anthropologique de ces tombes a èté faite par M"'r" J. Williams. L'estimation de l'àge a été fondée sur le degré d'usure des dents, la jonction basi-sphénoïde, la symphyse pubienne et l'apparence générale du squelette. Dans le cas de la tombe 1, le degré de fusion des épiphyses d'os long a permis une estimation plus précise de l'àge, ainsi que pour le squelette bien conservé du petit enfant de la tombe 5 , avec le recouvrement de quelques-unes des dents temporaires. Seul un
La vocation de zone funéraire est particulièrement marquée dans la pièce $\mathrm{P} 4$ et la partie immédiatement mitoyenne de l'espace P10, le long du mur 15 , qui contiennent respectivement un et quatre squelettes.

Dans la pièce 4, la tombe 2 , en pleine terre, est installee dans la fosse B (fig. 40) creusée dans le béton de tuileau. Le sujet, orienté est-ouest, tenait

adulte avait un bassin complet. En l'absence de symphyse pubienne et de la grande échancrure sciaticque, la détermination du sexe a été effectuée par le critère de gracilité ou de robustesse des traits caractéristiques de tout le squelette. les huit tombes de la Porte d'Oree comprennent un enfant de 16 a 18 mois (T5), un adolescent age de 18 a 20 ans (T1) et six adultes âges de 25 a 15 ans environ (T2 à T4. T6 a T $T$ ). Deux des adultes etaient de sexe masculin. un etait probablement de sexe masculin et un de sexe féminin (T6). Leur stature (mis a part la femme, qui mesurait $1.56 \mathrm{~m}$ ) variait de $1,7.2 \mathrm{~m}$ a $1,76 \mathrm{~m}$. Trois des adultes sembleraient ètre des dolichocephales. Ieetude anthropologique complete de ces tombes doit paraitre prochainement (I)utour, Berato, Williams, 1991). 
dans sa main un fragment de panse d'amphore du type Late Roman Amphora 1 qui recolle avec un autre tesson retrouvé en stratigraphie dans cette mème pièce.

Dans l'espace P10, les squelettes 5 et 6 sont ceux d'un enfant et d'une femme (peut-être sa mère), réunis dans le même cercueil dont nous avons trouvé les clous régulièrement répartis sur le pourtour. La sépulture, creusée à partir de la surface 8 , était orientée est-ouest, comme la tombe 4 dont seuls les os des membres inférieurs étaient conservés. Le squelette de la tombe 7 est plus complet ; il avait semble$\mathrm{t}$-il été déposé dans une fosse en pleine terre, orientée est-ouest et creusée à partir de la surface 9. C'est peut-être à cet ensemble funéraire qu'il convient de rattacher l'inhumation 8 retrouvée dans l'espace $P 6$, secteur de la fouille où la stratigraphie était extrêmement perturbée par des remaniements modernes et contemporains. Cette tombe creusée dans la couche इ était également orientée est-ouest, la tête du sujet venant s'appuyer contre le mur 32. Le squelette reposait à même le sol et des fragments de tuiles plates, disposés de chant sur les côtés, étaient sûrement les témoins d'un coffrage ou d'une bàtière de tuiles.

\section{Éléments de datation}

A l'exception de la tombe 3 qui a livré deux fragments de sigillée claire I) et un tesson de sigillée tardive estampée (DSP), les sépultures contenaient uniquement de la céramique commune ou résiduelle.

Le matériel présent dans la tombe 3 et sa position stratigraphique (elle est scellée par la couche 6 qui se place vers la fin du $\mathrm{V}^{\prime \prime}$ s. ou dans le courant du $\mathrm{vi}^{\mathrm{F}} \mathrm{s}$.) permettent de la dater du $\mathrm{v}^{\mathrm{c}} \mathrm{s}$. C'est également le cas de la tombe 7 , creusée dans la couche 9 et recouverte par la couche 8 du dépotoir. En revanche, les tombes 4,5 et 6 creusées dans la couche 8 sont recouvertes de différentes couches de remblais qui n'ont pas livré de matériel; cependant, il est probable qu'elles soient immédiatement postérieures à la tombe 7 , dans la mesure où elles présentent les mêmes caractéristiques.

\section{ÉTAT IVC : LE DÉMANTÈLEMENT DU BÂTIMENT}

Après le $\mathrm{vl}^{\mathrm{e}}$ s., le bàtiment est définitivement abandonné et les gravats de la couche de destruction s'accumulent sur les bétons de tuileau des pièces $\mathrm{P} 4$ et Pll et dans les salles et espaces voisins.

Les structures sont alors peu à peu démantelées afin d'en récupérer les matériaux. Seuls les blocs d'un assez gros module ont èté emportés. Ainsi, dans la pièce $\mathrm{P} 5$, la couche $3 \mathrm{~b}$, niveau de destruction constitué exclusivement de petites pierres et de mortier, résulte d'un tri minutieux auquel correspond une surface légèrement damée (S3a). La couche 4 contenait un fragment de DSP et un bord de Late Roman $C$ de la forme Hayes 3, tandis que la couche $3 \mathrm{~b}$ contenait un tesson de la forme Hayes 99 qui la situe à la fin du $v^{r} s$. ou dans le courant du $\mathrm{VI}^{\mathrm{e}} \mathrm{s}$.

Les structures récupérées à cette époque sont les murs 24, 35 et les deux parements qui ont remodelé tardivement l'espace interne de la pièce 4 (tranchées 29 et 30). Seules ces deux tranchées et la récupération du mur 24 ont livré un matériel abondant. La tranchée 29 ne contenait pas de sigillées claires D postérieures au milieu du ves. mais a livré un bord d'amphore du type Keay XXXV (fig. 44, $\mathrm{n}^{\circ} 6$ ). La tranchée 30 a fourni un bord de la forme Hayes 99 et, si l'on compare le matériel des deux tranchées, on constate des similitudes qui suggèrent nettement une récupération simultanée des murs à la fin $d u \mathrm{v}^{\mathrm{e}} \mathrm{s}$. ou dans le courant du $\mathrm{VI}^{\mathrm{e}} \mathrm{s}$. Le. comblement de la tranchée 28 (mur 24) est daté du $\mathrm{vI}^{\mathrm{F}} \mathrm{s}$. par deux sigillées claires I) (une forme Hayes 99, une forme Hayes 88) (tabl. VI, p. 221).

\section{ÉTUDE DE LA CÉRAMIQUE}

Ces niveaux de l'Antiquité tardive ont livré 6206 tessons dont $55 \%$ proviennent de la pièce 10 où seul le dépotoir (couches 8,9 et 10) a fait l'objet d'une étude plus précise. Dans la pièce P5, nous avons comptabilisé 1646 tessons et seulement 201 dans la pièce 4 où le béton antique avait été réutilisé. Les tranchées de récupération des quatre murs spoliés à la fin de l'Antiquité contenaient 719 tessons principalement issus des tranchées 28 (mur 24), 29 et 30.

Nous avons choisi de présenter ici les catégories de céramiques les plus représentatives pour cette période, il s'agit des sigillées claires $\mathrm{D}$, des sigillées tardives estampées (DSP) et des céramiques communes grises, brunes et claires. En effet, les céramiques luisantes sont rares, nous en comptons seulement neuf fragments dans le dépotoir et la Late Roman $C$ (sigillée phocéenne tardive) est représentée par un tesson de la forme Hayes 3 provenant de la pièce 4 (couche 3) (fig. 49A, no 1), ainsi que par un fragment de fond où figure un décor de clé (Hayes, 1972. $\mathrm{n}^{\circ} 26 \mathrm{c}, 440-490$ ) qui provient de la tranchée 29 (fig. $49 \mathrm{~B}, \mathrm{n}^{\circ} 2$ ). 

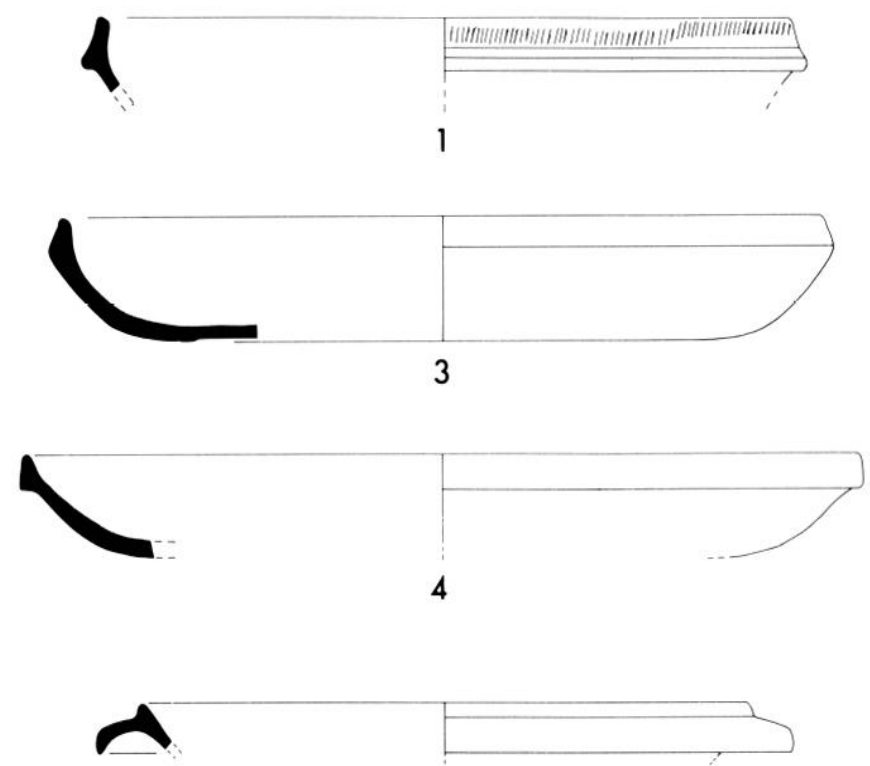

9

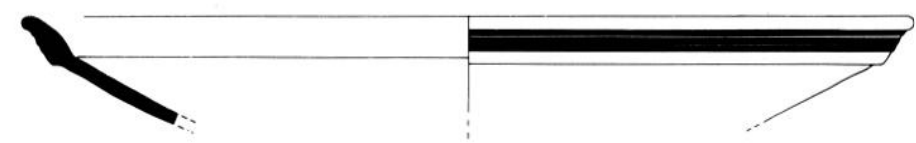

22

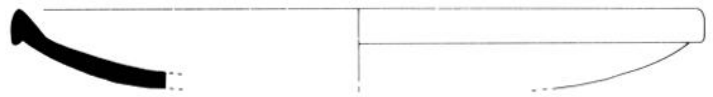

23

Fig. 49A - Late Roman C ( $\left.\mathrm{n}^{\circ} 1\right)$, sigillée claire D $\left(\mathrm{n}^{\text {os }} 3-7,9,11,12,14-23\right)$

Un graphique établi à partir du comptage global des tessons permet de constater l'importance relative de chacun des cinq groupes considérés (fig. 48). Si l'on compare les deux catégories de vaisselle fine, on est frappé par la faible proportion des DSP et la place importante occupée par la claire $D$ à la différence de ce que l'on a coutume d'observer sur les sites de Provence occidentale ou du Languedoc (CATHMA, 1984, p. 37, fig. 5). Parmi les céramiques communes, les pâtes brunes sont mieux représentées que les pâtes grises, caractéristique régionale déjà mise en évidence à Olbia ${ }^{22}$ ou à Toulon (Bérato et alii, 1986b, p. 159, fig. 20).

\section{La sigillée claire D}

Parmi les sigillées claires africaines, les claires I) sont majoritaires et fournissent pour tous les

22 Fouilles effectuees par M. Bats, renseignement fourni par J. Andreau.

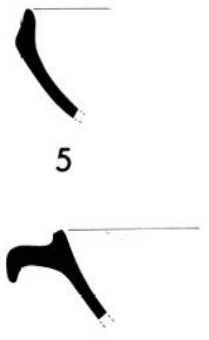

11
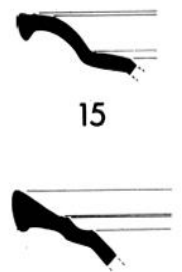

18

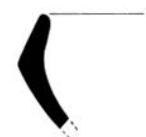

6

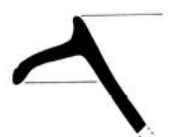

12
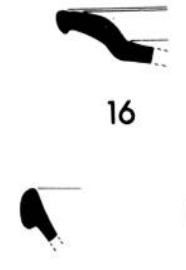

19

20

21 niveaux des éléments de datation. Un comptage réalisé à partir des bords a permis d'identifier 79 exemplaires parmi lesquels nous avons distingué onze formes répertoriées suivant la typologie de J.W. Hayes. Cette catégorie occupe une place importante dans le dépotoir et dans le foyer de la pièce $\mathrm{P} 4$, elle est moins bien représentée dans la pièce P5̆ (tabl. VII, p. 221).

\section{État IVA}

Dans l'espace P10 (couches 8, 9 et 10), la plupart des céramiques claires $D$ appartiennent à des formes de la première moitié $d u v^{e} s$. à l'exception toutefois d'un tesson de la forme 99 qui constitue peut-être une intrusion parmi un matériel par ailleurs très homogène ${ }^{23}$ (fig. $49 \mathrm{~A}, \mathrm{n}^{\circ} 19$ ).

La forme Hayes 6113 est bien représentée avec quinze objets dans la couche 8 , deux dans la couche 9 et un dans la couche 10 (fig. 49A, nos 3-7). Cette forme de la première génération figure également en bonne place à Marseille en période 1 (Bonifay, 1983 , p. 108, fig. 17). Les autres vases se rattachent à cette même phase de production, il s'agit des formes Hayes $91 \mathrm{AB}$ (fig. $49 \mathrm{~A}$ et $\mathrm{B}, \mathrm{n}^{\mathrm{os}} 8-12$ ), Hayes 59B (fig. 49B, no 13), Hayes 67 (fig. 49A,

23 Il faut cependant signaler que cette forme est attestée à Conimbriga dès 465)-468 (Carandini, 1981, p. 109). 

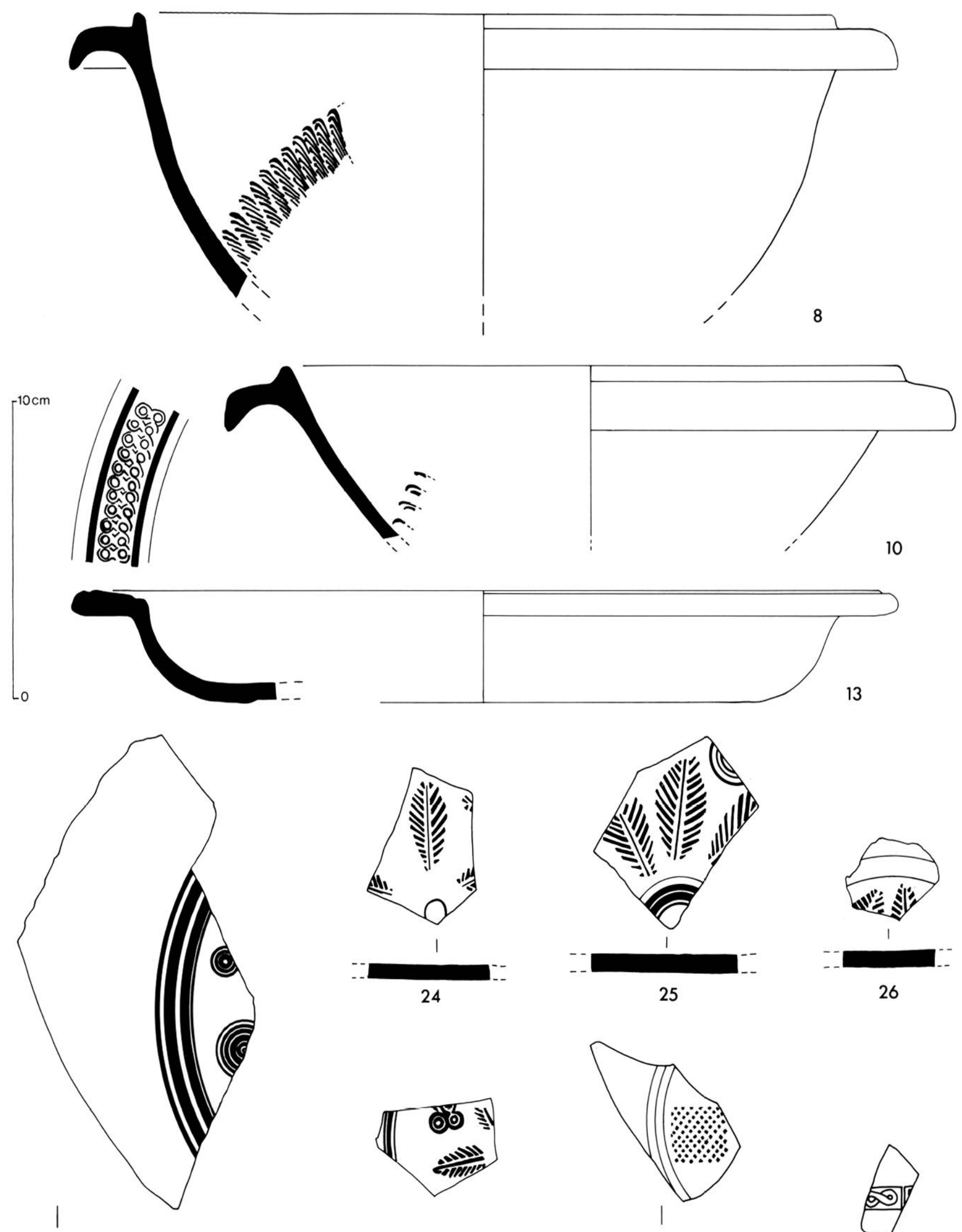

13
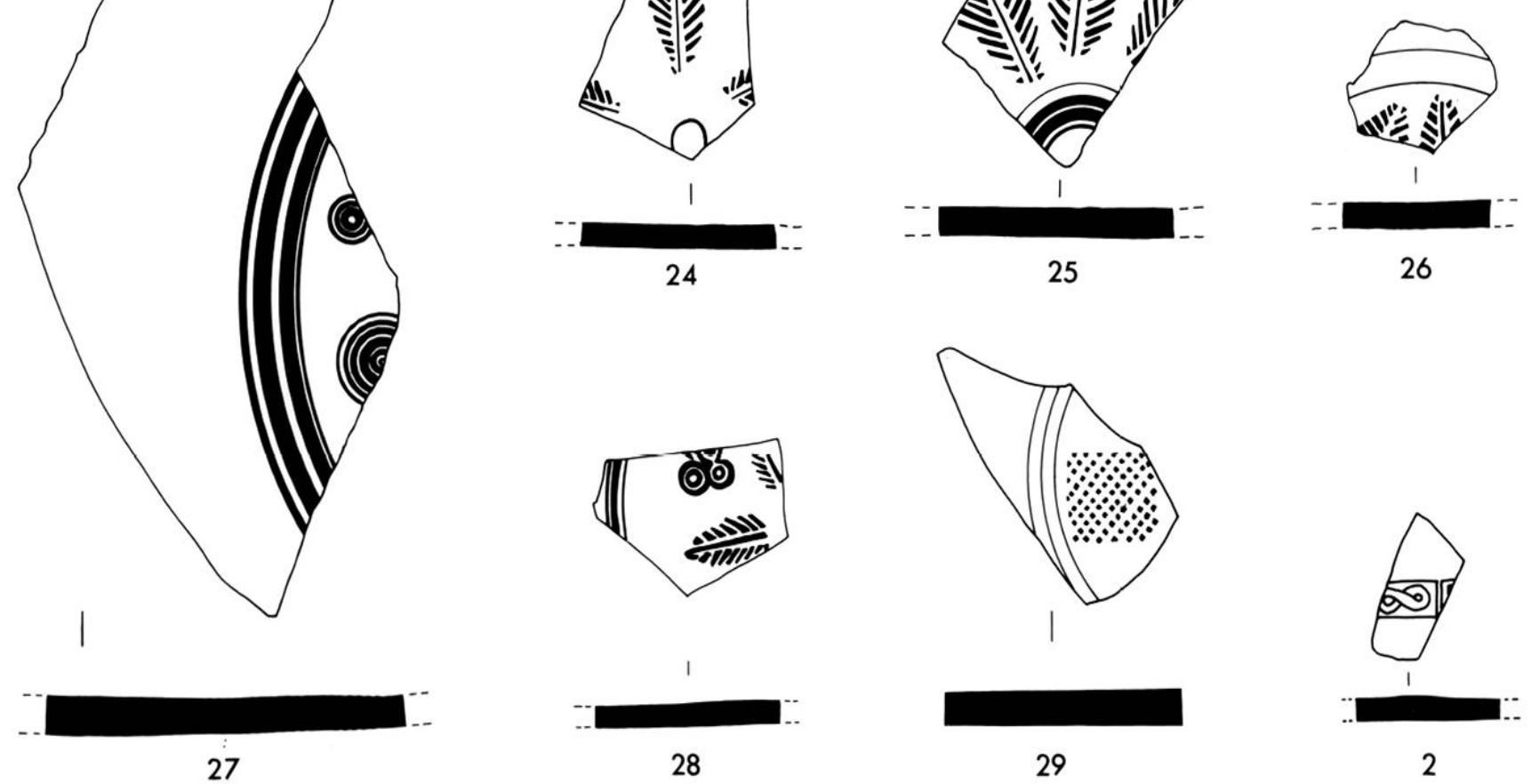

Fig. 49B - Late Roman C $\left(n^{\circ} 2\right)$, sigillèe claire D ( $\left.n^{\circ s} 8,10,13,24-29\right)$ 
$\mathrm{n}^{\text {os }}$ 14-17), Hayes 69, Hayes 58, Hayes 68 (fig. 49A, $\left.n^{\circ} 18\right)$. On compte par ailleurs un tesson de la forme Hayes 73 daté entre 420 et 475 . D'autres fragments, dont les décors appartiennent au style $A$ de J.W. Hayes, se placent dans la même fourchette chronologique ${ }^{24}$ (fig. 49B, $\mathrm{n}^{\text {os }}$ 24-26).

Dans la pièce $\mathrm{P} 4$ les céramiques claires D provenant du foyer A se raltachent toutes aux formes de la première moitié du ve $s$. : trois formes Hayes $61 \mathrm{~B}$, trois formes $59 \mathrm{~B}$ et une forme $91 \mathrm{AB}$.

Les éléments de décor corroborent cette datation : fragment de fond de grand plat (fig. 49B, $\left.n^{\circ} 27\right)$ orné de cercles concentriques (Hayes, 1972, fig. $40, n^{\circ} 26$ e ou f, style $A$ (ii)-?(iii) et fig. $40, n^{\circ} 29,1$ style $\mathrm{A}(\mathrm{ii}))$; fragment de fond décoré d'un trèfle (Hayes, 1972, fig. 43, no $83 \mathrm{~h}$ style $\mathrm{A}(\mathrm{ii})$-(iii)) associé à une palmette (fig. 49B, n ${ }^{0} 28$ ).

\section{État IVB}

Dans les autres contextes, les céramiques claires D de la première génération sont toujours présentes mais on note l'apparition de productions postérieures.

Ainsi dans les fosses $\mathrm{C}$ et $\mathrm{Cb}$ de la pièce 4 , nous avons recueilli deux bords de la forme Hayes 88 qui apparait au début du $\mathrm{vi}^{\mathrm{e}} \mathrm{s}$.

C'est également à la fin du v"s. ou au début du $\mathrm{vi}^{\mathrm{e}} \mathrm{s}$. que nous plaçons le dernier niveau d'occupation de la pièce 5 (couche 5), qui contient un tesson de la forme Hayes 99, ainsi que les niveaux immédiatement postérieurs (S4 et S3b) qui livrent chacun un tesson de cette même forme (fig. $49 \Lambda, \mathrm{n}^{\circ} 20$ ).

\section{État IVC}

Dans la tranchée 29 , le seul élément de datation nous est fourni par un fragment de fond qui présente un décor de carré résillé du style A(ii)-(iii) (Hayes, 1972, fig. 42, no 69) (fig. 49B, no 29). En revanche, on compte un tesson de la forme Hayes 99 dans la tranchée 30 ainsi que dans la tranchée du mur 24 (fig. $49 \Lambda, n^{\circ} 21$ ) où il est associé à une forme 88 (fig. $\left.49 \mathrm{~A}, n^{\circ} 22\right)$ (il s'agit d'une variante présentant des cannelures à l'extérieur) et à deux formes $87^{25}$ (fig, 49A, n' 23).

21 Ces fragments, appartenant a des fonds de plats. sont ornés de palmettes, la palmette de la fig. 49, n" 25, associée a des cercles concentriques, peut etre identities à la figure 38, n" 4i de la typologie de J. W. Ilayes (style Aii, 350. 420).

25) La forme 87 fait son apparition sur différents sites vers la fin du v"s. et le début du vi" s. (CATIIMA, 1984, p. 36).

\section{Les Dérivées de Sigillées Paléochrétiennes (DSP)}

Cette catégorie est mal représentée sur le site où l'on ne répertorie que trente-huit tessons dont treize présentent un décor estampé tandis qu'un seul fragment est orné de guillochis (fig. $\left.50 \mathrm{~B}, \mathrm{n}^{\circ} 11\right)^{26}$.

Les pâtes étaient en général de couleur gris clair, contenant parfois une grande quantité de particules de mica, et la surface dépourvue d'engobe à l'exception d'un bol de la forme Rigoir 3a recouvert d'un engobe noir et brillant (fig. $50 \mathrm{~A}, \mathrm{n}^{\circ} 3$ ).

Cependant, un bol de la forme Rigoir 6c (Rigoir, 1985) (fig. 50A, $\mathrm{n}^{\circ} 5$ ), particulièrement bien conservé, ainsi que cinq tessons d'un vase à liquide se caractérisent par une pâte fine et dure, de couleur gris foncé recouverte d'un engobe noir et brillant.

Un fragment de fond (provenant de la tranchée 28) orné d'une palmette présente une pâte micacée dont la couleur orangée serait le résultat d'une cuisson mal menée (fig. 50B, $\mathrm{n}^{\circ} 16$ ).

L a plupart des tessons observés appartiennent à des formes de vaisselle (bols, coupes ou assiettes), les formes utilitaires ne sont représentées que par un bord de mortier de la forme Rigoir 38 (1985, fig. $48 \mathrm{~B}, \mathrm{n}^{\circ} 10$ ).

\section{État IVA}

Deux bords d'assiettes de la forme Rigoir 1 proviennent du dépotoir P10 (couche 8), l'un d'eux, de production marseillaise, est décoré de rouelles du type Rigoir 24 (fig. 50A, no 1) (Rigoir, 1960).

Deux bols de la forme Rigoir 3a sont issus de cette même couche, l'un d'eux est orné de rouelles (poinçon local) (fig. 50A, $n^{\circ} 2$ ) l'autre de palmettes en fer de lance (peut-être poinçon Rigoir $\mathrm{n}^{\circ}$ 112) (fig. $50 \mathrm{~A}, \mathrm{n}^{\circ} 3$ ).

Un bol hémisphérique de la forme Rigoir $6 c$, probablement de production marseillaise et qui, nous l'avons vu, se caractérise par l'excellente qualité de sa pâte et de son engobe, présente un décor d'une extrême finesse composé de palmettes en feuilles de laurier, $\mathrm{n}^{\circ} 165$, qui se chevauchent (fig. $50 \mathrm{~A}, \mathrm{n}^{\circ} 5$ ).

Un fragment de panse de bol est orné de palmettes ovales surmontees d'arceaux en double pointillé (fig. $50 \mathrm{~A}, \mathrm{n}^{\circ} 6$ ).

Un fond de grand plat présente un décor de carrés concentriques inscrits dans un ovale irrégulier (poinçon local), associé à des palmettes (fig. 50B, n"15).

26 Pour l'étude de ce materiel, nous avons beneficié de l'aide de J. et Y. Rigoir. 

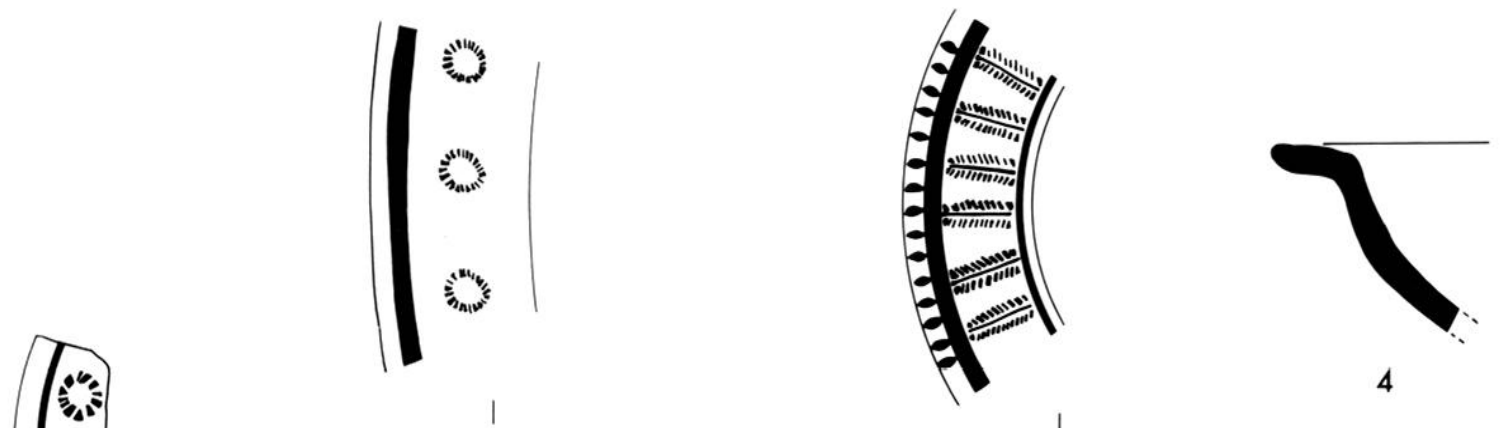

4
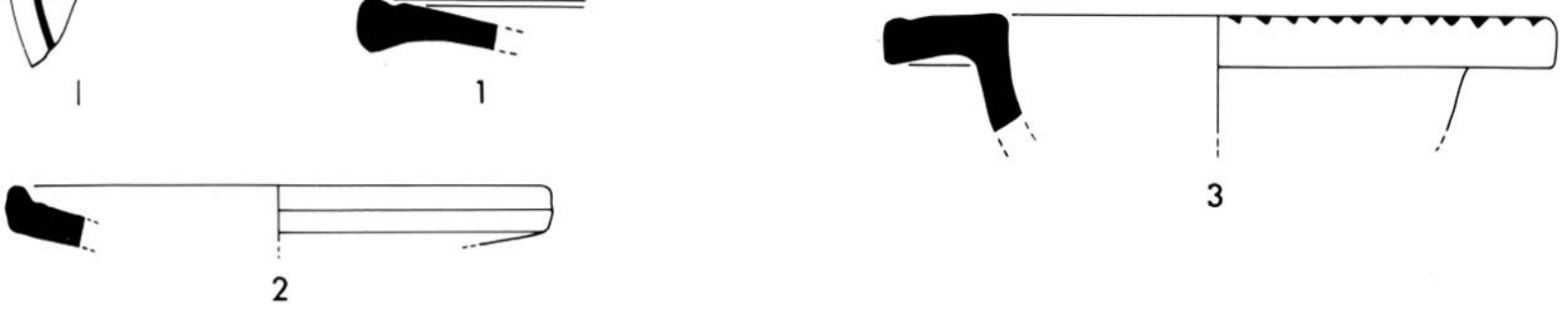

3
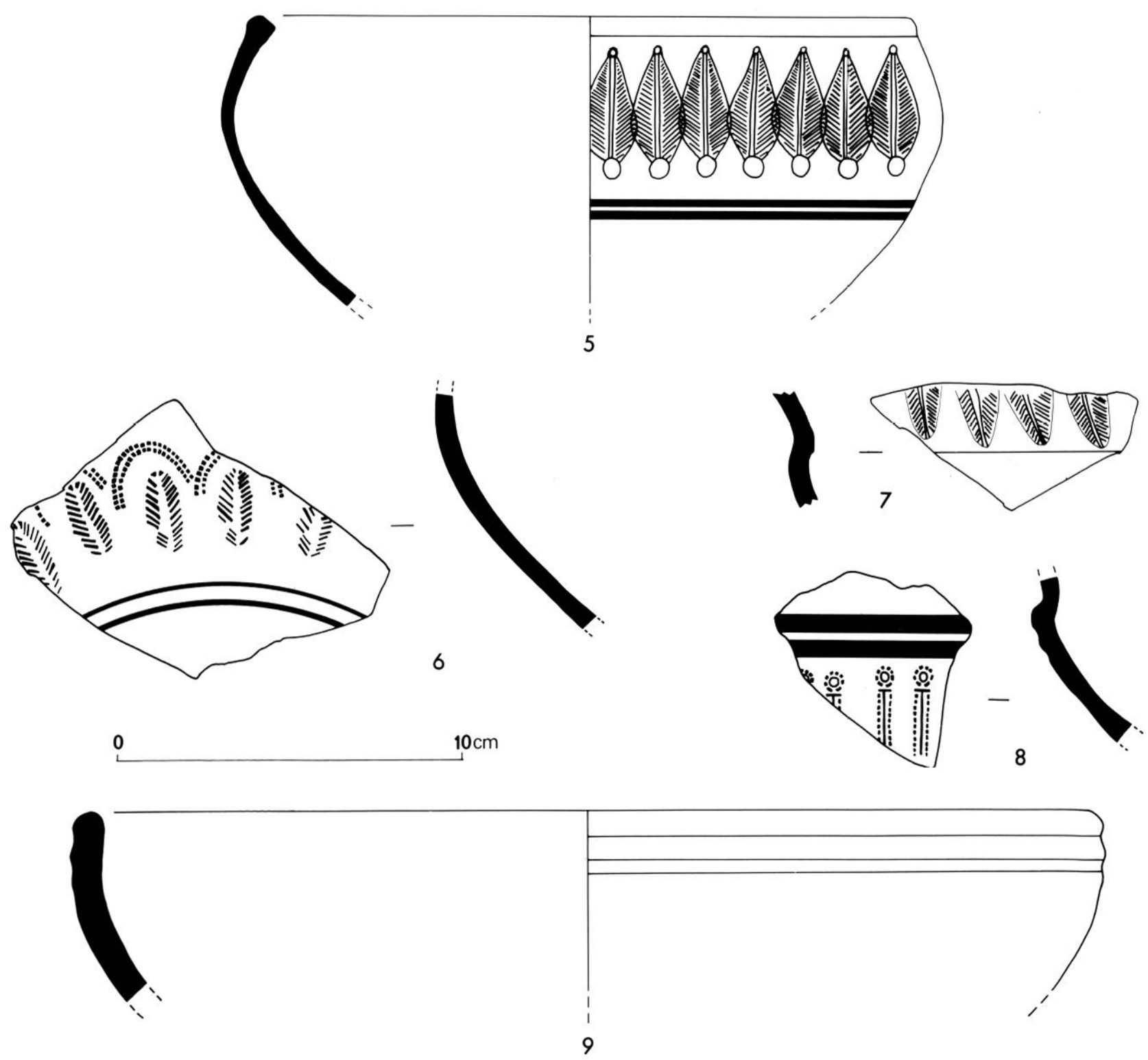

Fig. 50A - Dérivées des sigillées paléochrétiennes (DSP) (n 1-9). 

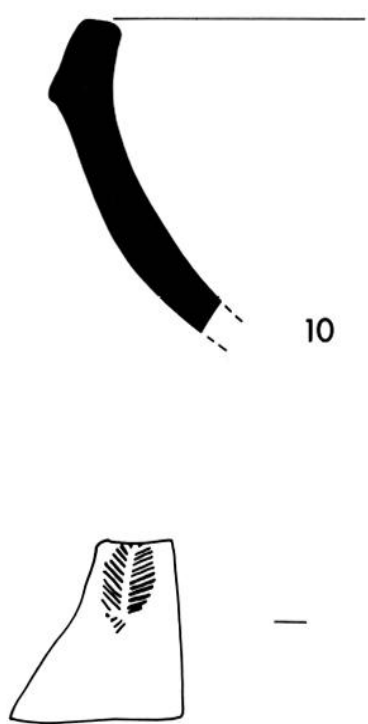

13

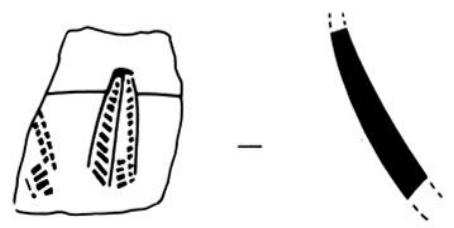

14

0 $10 \mathrm{~cm}$

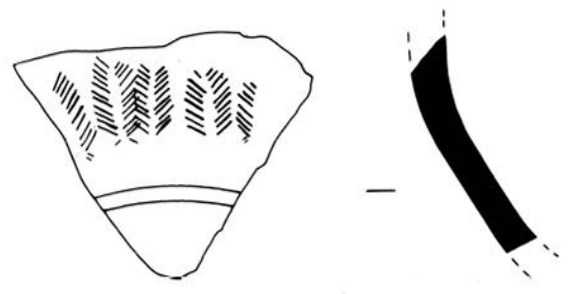

12

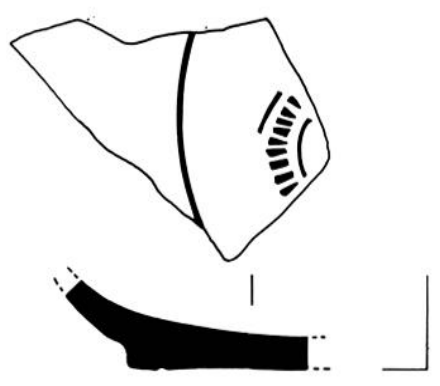

16

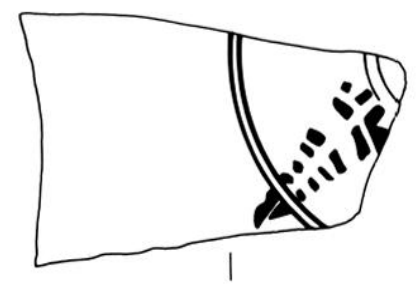

17

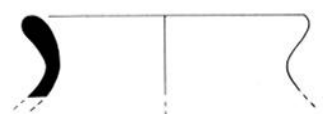

18

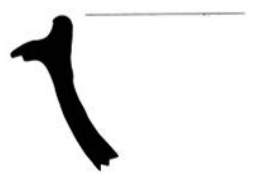

21

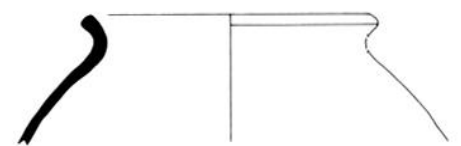

19

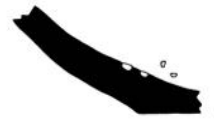

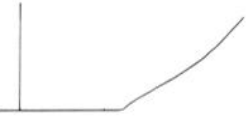

22
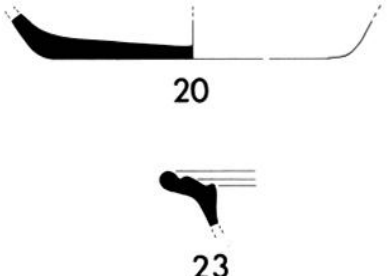

논 $10 \mathrm{~cm}$

Fig. 50B - Dérivées des sigillées paléochrétiennes (DSP) ( $\left.\mathrm{n}^{\mathrm{is}} 10-17\right)$; communes grises ( $\left.\mathrm{n}^{\natural 4} 18-23\right)$.

Des poinçons de palmettes, motif le plus fréquemment rencontré sur le site, ornent également deux tessons de bols ou de coupes provenant du dépotoir (fig. 50B, $\mathrm{n}^{\circ \mathrm{s}} 12$ et 13 ).

Dans la pièce $\mathrm{P} 4$ le foyer $\mathrm{A}$ contenait seulement deux fragments de DSP dont un de la forme Rigoir 18 orné de palmettes en losange (fig. 50A, n" 7).

\section{État IVB}

Dans la pièce $\mathrm{P} 5$, la couche $6 \mathrm{~b}$ a livré un bord de la forme Rigoir 2-3 exempt de décor (fig. 50A, $\mathrm{n}^{\circ} 4$ ), ainsi qu'un fragment informe orné de palmettes (fig. 50B, $\mathrm{n}^{\circ} 14$ ); le seul tesson de cette catégorie figurant dans la couche 4 appartient à une forme 
Rigoir 18 ornée de colonnettes ( $n^{\circ} 96$ ), de production marseillaise (fig. $50 \mathrm{~A}, \mathrm{n}^{\circ} 8$ ).

\section{État IVC}

Les trois formes provenant des tranchées de récupération des murs ne sont pas décorées, il s'agit d'une forme Rigoir 2-3, d'une forme Rigoir 4 provenant de la tranchée 28 et d'une forme Rigoir 4 découverte dans la tranchée 30 (fig. $50 \mathrm{~A}, \mathrm{n}^{\circ} 9$ ).

De manière générale, l'origine des tessons, marseillaise ou locale, a pu être établie; cependant un fragment issu du dépotoir n'a pu être clairement identifié : il appartient à un fond de grand plat, présente une pâte micacée de couleur beige orangé et est orné d'une palmette. Peut-être s'agit-il d'une importation dont la provenance reste encore indéterminée.

Le hiatus mis en évidence à travers l'étude des sigillées claires D entre les niveaux de l'état IVA et IVB semble confirmé dans la mesure où les formes représentées dans le dépotoir et le foyer sont typiques du ve s. (CATHMA, 1984, p. 41, fig. 8), tandis que les décors se raréfient dans les niveaux postérieurs ${ }^{27}$, mais il est évidemment difficile de tirer des conclusions définitives à partir d'une quantité si réduite de tessons.

\section{Céramique commune grise}

Dans le dépotoir et la pièce 4 , ce groupe est peu important. En revanche, il est bien représenté dans la pièce 5 qui regroupe $49,65 \%$ des tessons comptabilisés, ainsi que dans les tranchées de récupérations de murs (dix-huit tessons) et dans les fosses C et Ca (dix-sept tessons).

L'essentiel des formes identifiables d'après les bords sont des formes fermées ou ollae à fond plat (fig. 50B, $\mathrm{n}^{\circ} 20$ ) et bord évasé à lèvre simple (fig. $50 \mathrm{~B}, \mathrm{n}^{\text {os }} 18$ et 19) que l'on peut comparer à certaines formes de la fin du ve $v^{e}$. ou du début du $v^{e}{ }^{e} s$. trouvées à la Gayole (Var) (CATHMA, 1984, p. 48, fig. 12).

On note également la présence dans le dépotoir d'un fond de mortier (fig. 50B, no 22) et d'un bord de mortier du type D (Pelletier, Vallauri, à paraître), ainsi que d'un marli dont la pâte fine et le décor de. cannelures dénotent une bonne qualité de fabrication.

Il est nécessaire de préciser que cette étude porte sur un nombre restreint de fragments réellement identifiables, ce qui limite l'intérêt d'une ana-

27 A Marseille, le nombre de tessons décorés diminue dans les niveaux postérieurs au $v^{e} s$. (Cavailles-Llopis, 1986, p. 179 , fig. 16). lyse plus poussée d'un groupe qui apparaît comme nettement sous représenté sur le site à l'instar de ce qui a pu être constaté sur d'autres sites de Provence orientale.

\section{La céramique commune brune}

Nous avons réuni dans cette catégorie un ensemble de céramiques culinaires à pâte rouge orangé, granuleuse, contenant des particules blanches et du mica dans des proportions variables et dont la surface extérieure est toujours cendrée. Cette pâte bien cuite et sonnante est d'assez bonne qualité malgré son aspect un peu grossier. Des vases possédant les mêmes caractéristiques avaient déjà été mis en évidence sur le site de Saint-Blaise (CATHMA, sous presse, fig. 31) et cette production est ici suffisamment bien représentée pour nous permettre de proposer une première typologie qui regroupe essentiellement des pots à cuire et des couvercles.

\section{Marmites à profil globulaire}

Il semble possible de rattacher un de ces vases, de facture assez fine et dont l'anse est accrochée sous le rebord, à une variante du type 13 de la CATHMA (fig. $51 \mathrm{~A}, \mathrm{n}^{\circ} 1$ ). Ce type de petites marmites, bien diffusé sur les sites côtiers (Marseille, Saint-Blaise ...) proviendrait de Ligurie.

Un second vase globulaire (fig. $51 \mathrm{~A}, \mathrm{n}^{\circ} 2$ ) à panse cannelée se singularise par sa grande taille et par sa forme proche du type CATHMA 3, cependant sa pâte, qui a subi un excès de cuisson, ne permet pas d'attester une origine africaine, il pourrait en effet s'agir d'une imitation (peut-être ligure).

Les autres marmites appartiennent à un groupe homogène, elles sont de petite taille (le diamètre d'ouverture est généralement compris entre 14 et $18 \mathrm{~cm}$ ) et munies de deux anses étroites et épaisses qui s'accrochent sur le rebord. Les fonds qui peuvent être associés à ces vases sont plats, la liaison avec la panse bien marquée (fig. $51 \mathrm{~B}, \mathrm{n}^{\text {os }} 27$ et 28). Les rebords peuvent être soit simplement évasés (fig. 51A, nos $3-10$ ) soit dotés d'une gorge assez grossièrement façonnée (fig. $51 \mathrm{~A}, \mathrm{n}^{\mathrm{os}} 11-18$ ).

Nous avons comptabilisé 57 bords de ce type de marmites :

P4 couche 6 fosse $\mathrm{Cb}$ (1 ex.); P4 tranchée 28 (1 ex.); P4 tranchée 29 (18 ex.); P5̃ C6c (1 ex.); P10 surface 3b (6 ex.); P10 C8 (21 ex.); P10 C9 (9 ex.).

\section{Marmites à profil caréné}

Nous avons comptabilisé cinq vases de ce type dans la couche 8 du dépotoir, dont une forme 


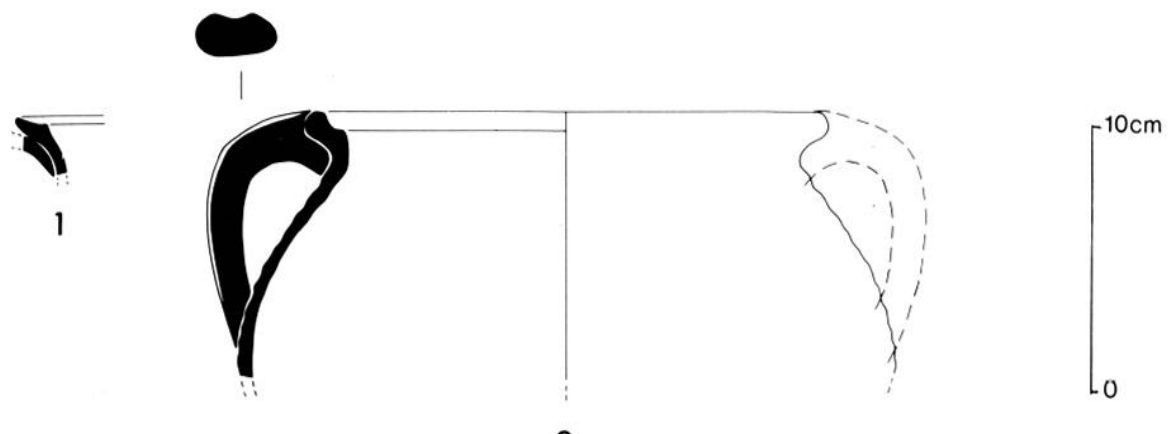

2
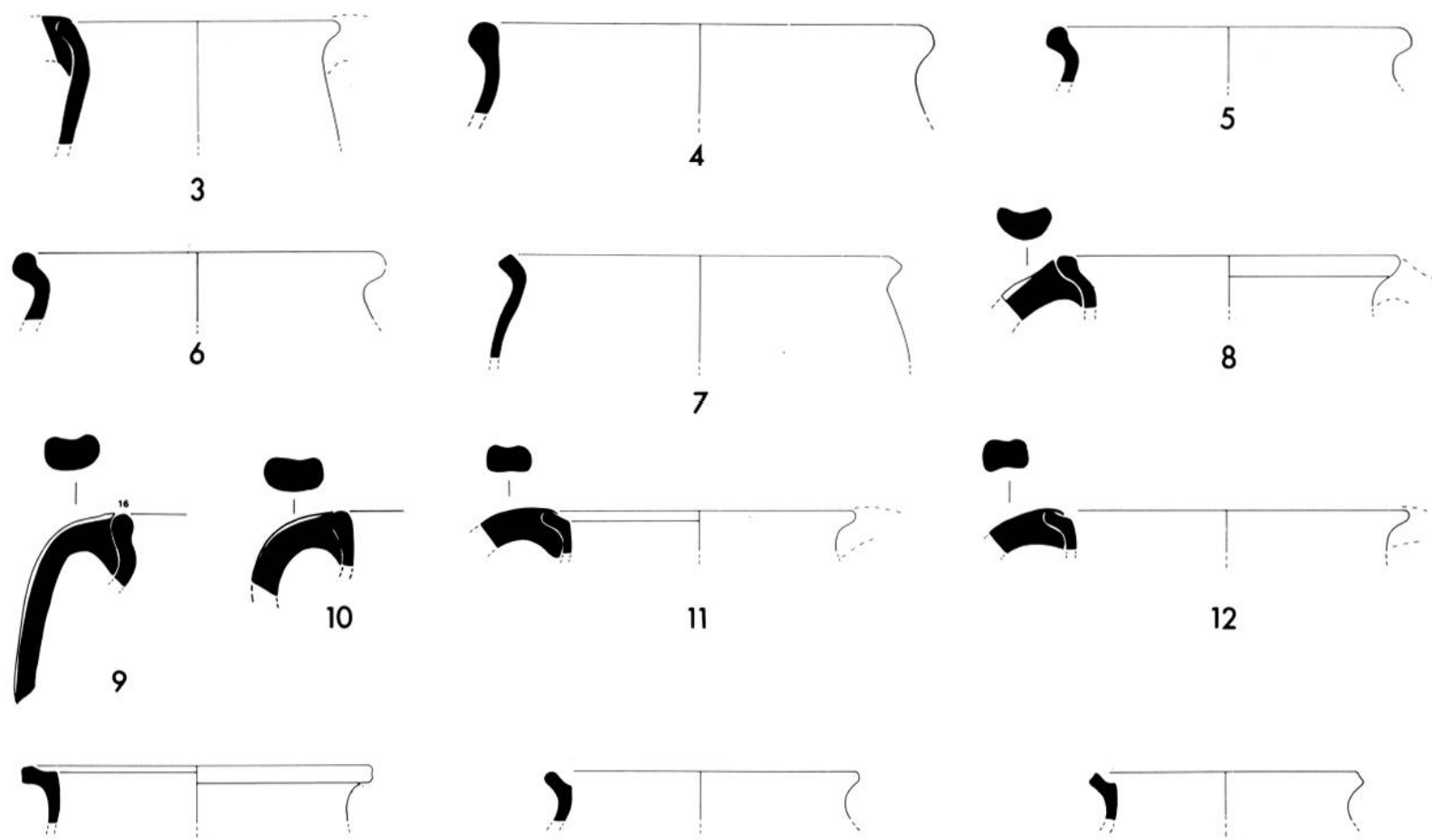

13

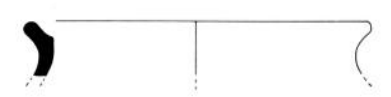

14

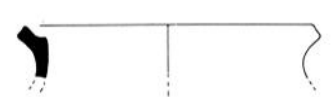

15

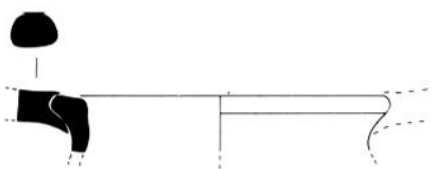

16

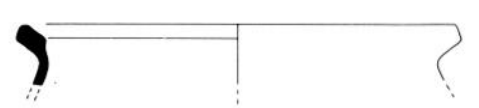

17

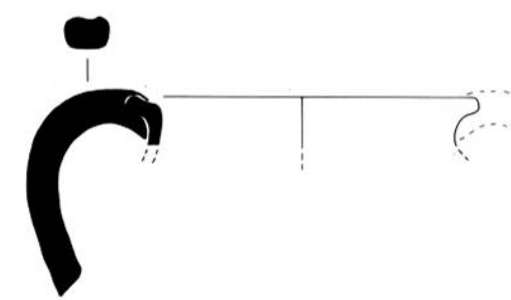

18
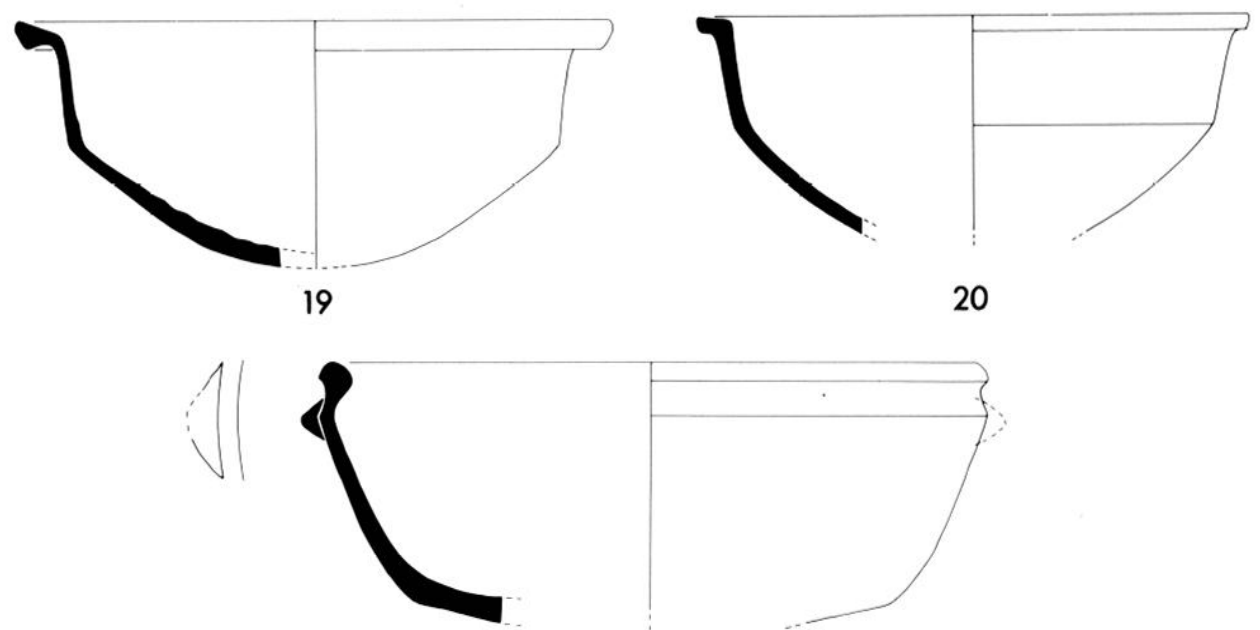

Fï. $51 \mathrm{~A} \mathrm{--} \mathrm{Communes} \mathrm{brunes} \mathrm{(n*s} 1-21)$. 


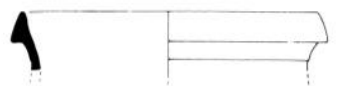

22

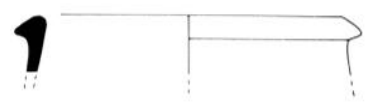

23

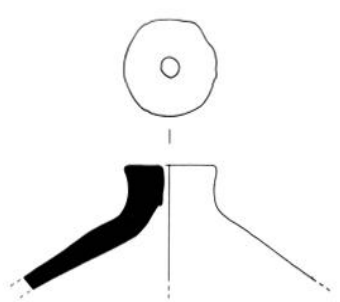

24

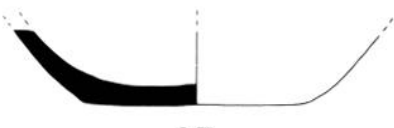

27

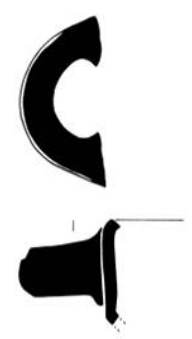

29

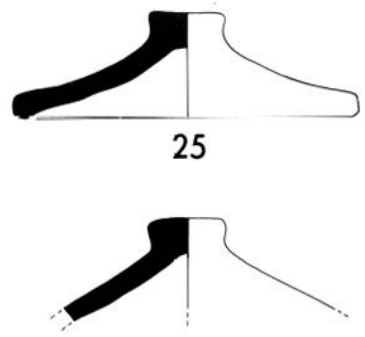

26

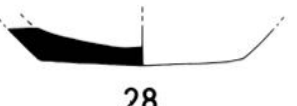

28

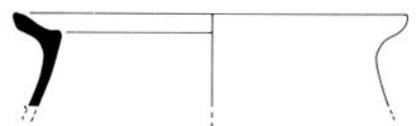

30

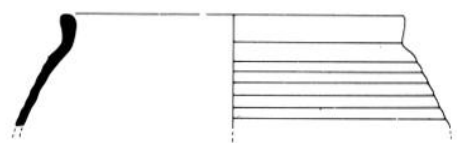

31
Fig. 51B - Communes brunes (nos 22-28), importations orientales et africaines ( $n^{\text {os }} 2931$ ).

presque complète. Ce récipient est large et peu profond ( $24 \mathrm{~cm}$ de diamètre pour $9 \mathrm{~cm}$ de hauteur pour l'exemplaire le plus complet); la lèvre, plus ou moins épaisse, est horizontale, la panse courte présente un profil presque rectiligne et se rattache au fond bombé par un angle bien marqué. C'est peut-être à cette forme qu'il conviendrait de rattacher deux autres bords provenant de la tranchée 30 et de la couche 8 du dépotoir (fig. $51 \Lambda$, n ${ }^{\text {ss }} 19$ et 20 ).

\section{Marmites dotées de deux poignées de préhension horizontales}

Deux vases provenant de la couche 8 du dépotoir et de la tranchée 29 se rattachent à cette forme large et peu profonde (diamètre : $26 \mathrm{~cm}$; hauteur : $10,5 \mathrm{~cm})$. Elle présente une lèvre arrondie, marquée à l'extérieur par un petit ressaut et légèrement inclinée vers l'intérieur, un profil tronconique, un fond bombé (fig. $51 \mathrm{~A}, \mathrm{n}^{\circ} 21$ ).

\section{Marmites à lèvre triangulaire}

Il est difficile de préciser le profil de ces marmites de petite taille (diamètre compris entre 12 et $14 \mathrm{~cm}$ ) dans la mesure ou elles ne sont représentées que par de petits fragments de bords provenant de la couche 10 du dépotoir et de la pièce 4 (Fosse Cb, tranchée '29) (fig. $51 \mathrm{~B}, \mathrm{n}^{0 \mathrm{~s}} \mathfrak{2 2}$ et 23 ).

\section{Couvercles}

Il s'agit de couvercles de forme tronconique (fig. $51 \mathrm{~B}, \mathrm{n}^{\text {os }} 24$ à 26) dotés d'un bouton de préhension (dans un cas percé d'un trou : fig. $51 \mathrm{~B}, \mathrm{n}^{\circ} 24$ ), dont les diamètres sont compris entre 14 et $21 \mathrm{~cm}$.

Nous en avons inventorié un dans la tranchée 30 , un dans la fosse 1 de la pièce 5 , sept dans la couche 8 et deux dans la couche 9 du dépotoir.

\section{Importations orientales et africaines}

Ce groupe ne figure pas dans nos histogrammes, il est en effet très peu important sur le site. Nous présentons néanmoins ici les différents types rencontrés :

- un exemple d'importation d'origine orientale provient de la couche 8 du dépotoir : il s'agit d'une forme de casserole à bord coupé et anse horizontale à pâte rouge feuilletée, du type CATHMA 4, forme $A$, déjà reconnue à Fréjus et datée pour le Midi de la France entre le ve s. et le viI ${ }^{e}$ s. (Vallauri, à paraître, fig. 44) (fig. $\left.51 \mathrm{~B}, \mathrm{n}^{\circ} 29\right)$;

- un autre bord de marmite (fig. $51 \mathrm{~B}, \mathrm{n}^{\circ} 30$ ) appartient au type CATHMA 5 d'origine égéenne (CATHMA, sous presse, fig. 17 et 18).

Les importations africaines sont mieux représentées, il s'agit en particulier des casseroles de la forme Hayes 197 : P5 fosse 1 (1 ex.), P10 C8 (1 ex.), P10 C9 (2 ex.) et des couvercles Hayes $196:$ tranchée 30 ( 1 ex.), P10 C8 (4 ex.), P10 C9 (4 ex.).

La couche 9 du dépotoir contenait également une forme de pot à cuire à pàte rouge bien cuite et surface cannelée qui est probablement aussi d'origine africaine ou orientale (fig. $51 \mathrm{~B}, \mathrm{n}^{0} 31$ ).

\section{La céramique commune claire}

Cette catégorie représente une part importante du matériel dans tous les niveaux : $27 \%$ dans le dépotoir, $42,48 \%$ pour le niveau d'occupation de la couche 5 et $49,55 \%$ dans les récupérations de murs, mais elle est également présente sur le site pour les niveaux antérieurs de sorte que la première difficulté consistait à trier le matériel résiduel. Il n'a pas toujours été simple par la suite de définir des séries de vases parmi un matériel regroupant une grande variété de formes et de pâtes. En ce qui concerne les 

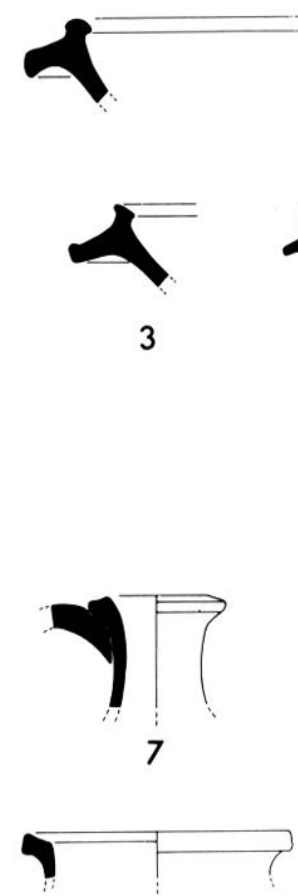

12
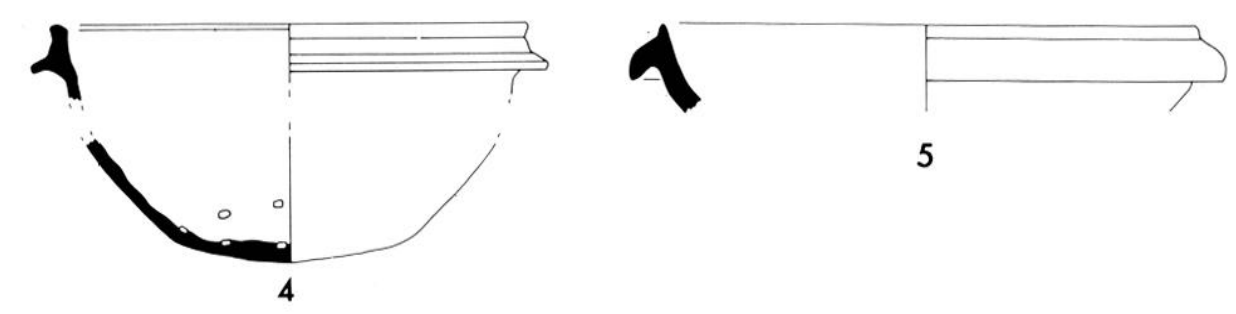

5

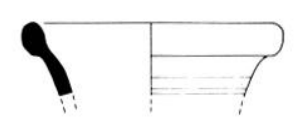

10

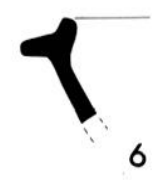

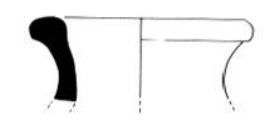

11

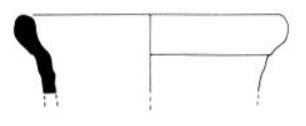

17

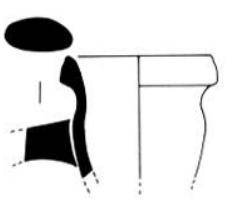

14

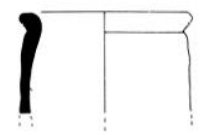

15

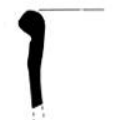

16

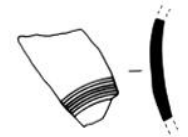

18

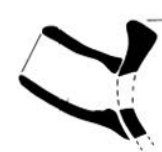

19

$10 \mathrm{~cm}$

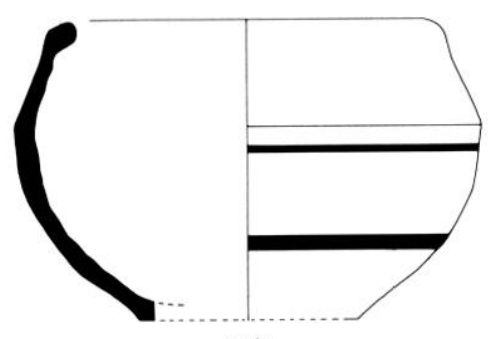

20
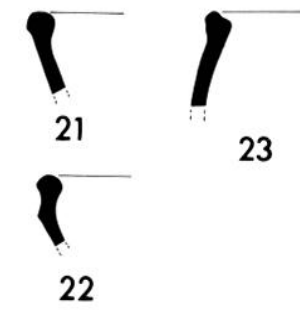

Fig. 52 - Communes claires. formes, mortiers et vases à liquides constituent deux séries importantes. Quand aux pâtes, en dehors des éléments d'origine africaine aucun groupe n'a pu être défini.

\section{Mortiers}

Quatre bords de mortiers issus du dépotoir possèdent une pâte orange et une surface blanchie au sel, ils se rattachent à la forme CATHMA type 1 , originaire d'Afrique du Nord et datée du $v^{e} s$. (fig. $52, \mathrm{n}^{\mathrm{os}} 1$ et 2) (CATHMA, sous presse, fig. 4 et 5). Un rebord à pâte orange granuleuse provenant du contexte $\mathrm{P} 5 \mathrm{C5}$ pourrait se rattacher à un même type de formes (fig. 52, $n^{\circ} 3$ ).

Un autre mortier (provenant de la couche $10 \mathrm{du}$ dépotoir), à pâte rouge fine et dont le fond présente des inclusions de couleur foncée, appartient peutêtre également à ce groupe des importations (fig. 52, $\left.n^{\circ} 4\right)$.
Neuf autres bords à pâte beige ou orangée sont plus probablement d'origine locale (fig. $52, \mathrm{n}^{\text {os }} 5$ et 6), ils proviennent des contextes suivants : P10 C9 (5 ex.); P4 C3 (1 ex.), P4 tranchée 30 (1 ex.), tranchée 28 (2 ex.). Deux des bords (très incomplets) provenant de la couche 9 du dépotoir étaient dotés de becs verseurs.

\section{Vases à liquides}

La catégorie des pâtes beiges ou orangées micacées regroupe la plus grande partie de ces vases parmi lesquels on distingue une grande variété de formes (fig. 52, nos 7 et 13).

D'autres tessons s'identifient peut-être à des importations d'origine africaine si l'on se réfère à l'aspect de leur pâte orangé foncé dont la surface est blanchie au sel (fig. $52, \mathrm{n}^{\mathrm{os}} 14$ et 17); cependant, il semble difficile de rattacher ces vases incomplets à des formes déjà répertoriées. Deux bords de cruches 
se rapprochent peut-être du type CATHMA 9-12 daté du v"s. (fig. 52, $\mathrm{n}^{\circ}$ 14) (Vallauri, à paraître, fig. 6,10 et 12). Un tesson de panse à pâte très claire, probablement de vase à liquide, orné de fines cannelures fait également partie des productions importées (fig. 52, no 18).

Parmi les formes plus rares, cilons un bord doté d'un bec tubulaire (P4 C3) (fig. 52, no 19), un bol en pâte de couleur orangée, bien cuite (tranchée 29) (fig. 52, n०20), deux bords de coupe en pâte micacée de couleur orangée (P10 C9 et P10 C8) (fig. 52, nos 21 et 22), un autre bord, peut-être de coupe, à pâte orangée contenant des particules blanches (P10 C8) (fig. 52, n*23) et un bol en pâte beige micacée (P10 C8) (fig. 52, n' 24).

Au terme de ce travail, une distorsion entre le matériel des états IVA et IVB a pu être mise en évidence, les céramiques à pàte grise prenant de l'importance dans les niveaux du $\mathrm{vI}^{\mathrm{e}} \mathrm{s}$. par rapport aux pâtes brunes. Mais l'ampleur de ce phénomène est difficile à préciser; révèle-t-il simplement un changement d'activité sur le site entre les deux périodes ou est-il le reflet d'un changement plus profond qui affecte les approvisionmements? Une comparaison avec d'autres ensembles proches et bien datés permettrait peut-être de trancher entre l'une ou l'autre de ces hypothèses.

Mais l'étude de ce matériel a permis de mettre en évidence un groupe important quantitativement et homogène constitué de céramiques culinaires qui se caractérisent par leur pâte (rouge orangé, granuleuse et contenant des inclusions blanches et du mica) ${ }^{28}$ et par leurs formes. Ce matériel est bien représenté dans le dépotoir $(39,72 \%$ des cinq catégories prises en compte), il est en revanche moins nombreux dans les niveaux de l'état IVB $(18,55 \%$ et $23,89 \%$ pour les deux phases de la pièce 5 , fig. 48 ).

Ce type de production a déjà été mis en évidence par I.. Vallauri à Saint-Blaise (Vallauri, à paraitre, fig. 20). On en ignore encore la provenance exacte; cependant l'abondance de ce matériel sur le site de Fréjus, comme d'ailleurs sur d'autres fouilles de Provence orientale (par exemple à Olbia), nous incite à penser que ces produits sont issus d'unc zone géographique proche ${ }^{29}$.

28 Les céramiques modelées du site, étudiées par $\mathrm{J}$. Bérato, sont parfois réalisées dans le mème type de pâte.

29 Une catégorie de céramiques culinaires à pàte brune a été mise en évidence sur les sites de Fréjus pour une période allant du $\mathrm{I}^{\mathrm{er}}$ au IV" s. par L. Rivet (1982).

\section{ÉTAT V}

L'abandon définitif du bâtiment public, qui va peu à peu disparaître du paysage urbain, s'étend à l'ensemble du site. Ce secteur de la ville connaît alors un déclin et sera délaissé pendant une longue période. En effet, dans la partie est nous ne décelons aucune trace d'activité avant l'époque moderne, tandis qu'à l'ouest la vocation du terrain est totalement modifiée à l'époque médiévale par sa transformation en une vaste aire de stockage.

Toute cette zone avait fait l'objet au II"s. d'aménagements qui ont consisté essentiellement en apport de matériaux destinés à établir un terre-plein et à la mise en place d'un drain (drain 21). Ce remblai est ensuite partiellement écrêté et le sommet de la canalisation devait être visible à l'époque médiévale. Ce qui restait de la couche 8 était recouvert de niveaux plus fins ne pouvant être associés à aucune trace d'activité tangible et probablement uniquement destinés à niveler le terrain afin de mettre en place la couche 3 . Unique niveau d'occupation pour l'époque médiévale, cette surface chaulée offre un aspect parfaitement plan, présentant un léger pendage est-ouest (fig. 5̃3).

Ce remblai, reconnu sur une superficie de plus de $300 \mathrm{~m}^{2}$ était percé de vingt-six fosses de forme régulière (fig. 53). Il n'est pas sans intérêt de constater que ces installations ont pris place sur une plateforme, zone parfaitement saine, à l'abri de la nappe phréatique, terrain tout à fait favorable au stockage de denrées et par conséquent à l'aménagement de silos.

Nous ne connaissons pas l'étendue exacte de cet espace reconnu, lors de l'enlèvement de la couche 8 , sur environ $500 \mathrm{~m}^{2}$ mais il s'étendait au-delà des limites de fouille vers le nord, le sud et l'ouest, ce qui indique que nous sommes en présence d'un aménagement important.

La répartition des fosses dans l'espace ne semble pas obéir à une règle stricte. Cependant, on décèle deux séries d'alignements (fig. 54); à l'est où la densilé est la plus imporlante, les fosses s'organisent en trois arcs de cercles orientés nord-est/sud-ouest (fosses $5,7,6 ; 2,8,21,9,11,1$ et 10,13,12,3) tandis qu'au nord-est de cette zone les silos sont alignés est-ouest de manière rectiligne (fosses relevées en limite de fouille et fosses $4,19,20)$.

La surface fouillée ne présentait aucune trace pouvant suggérer la présence d'un mur ou d'une cloison, excepté la tranchée (St 27), qu'il faut certainement identifier comme le résultat de la récupération 

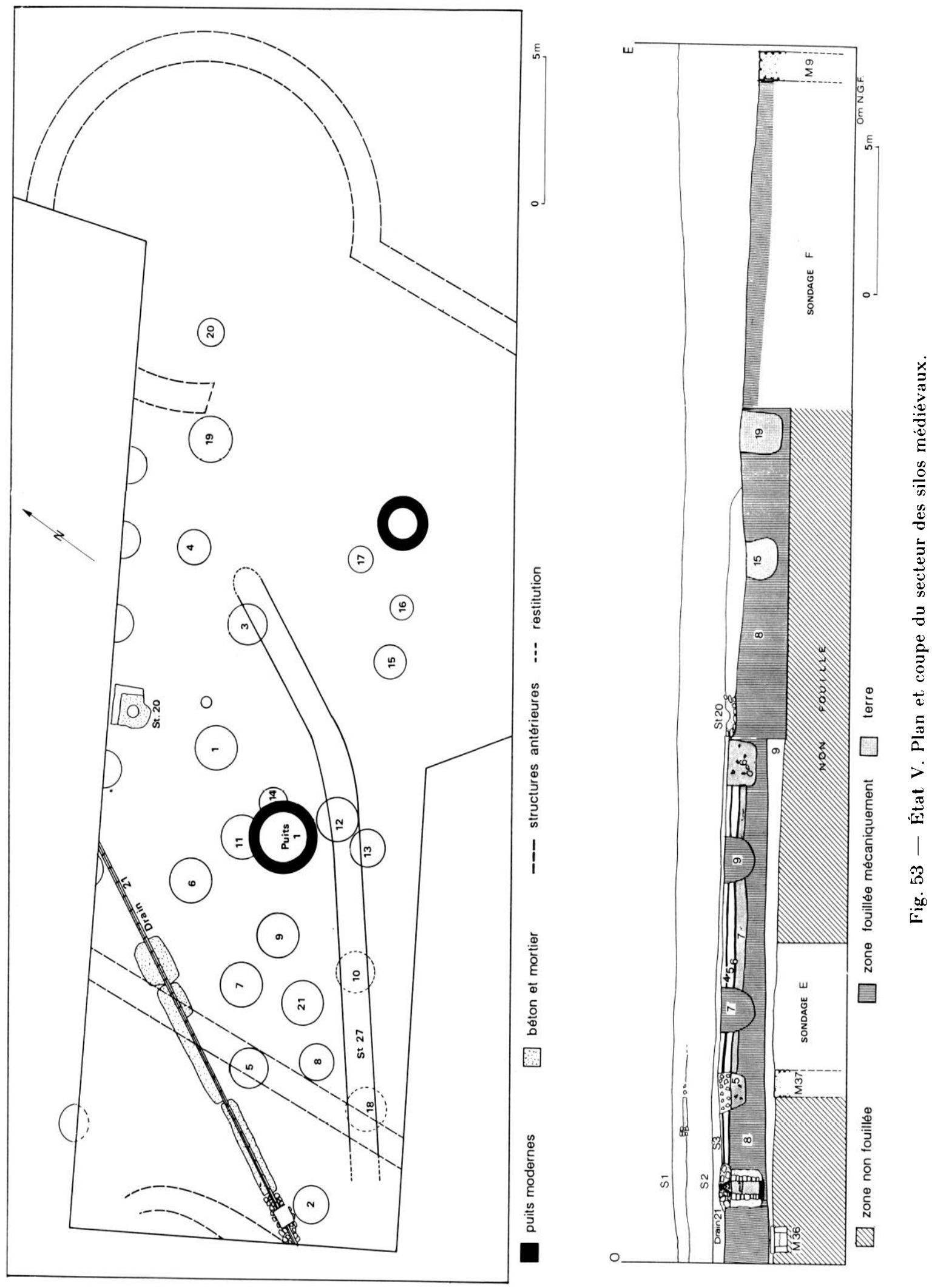
d'une structure : tout porte donc à croire qu'aucun de ces silos neetait couvert ${ }^{30}$.

\section{LES SILOS}

Ils possèdent un plan régulier et des dimensions assez constantes ; la profondeur des huit silos fouillés est toujours inférieure au diamètre d'ouverture ${ }^{31}$, leur contenance varie de $0,70 \mathrm{~m}^{3}$ à $1,70 \mathrm{~m}^{3}$; dans cinq cas elle est inférieure à $1 \mathrm{~m}^{3}$, capacité que l'on peut estimer relativement faible ${ }^{32}$. Ils offrent en général un profil cylindrique, à fond plat $(9,6,5$ et 1$)$ ou concave (3 et 7 ) (fig. 55). Cependant dans le cas des silos 4 et 15 , les parois se resserrent nettement vers le haut, de sorte que le diamètre d'ouverture est inférieur au diamètre maximum de la cavité. Il est. possible de penser que les surplombs des autres fosses aient pu s'effondrer, modifiant ainsi la forme initiale des excavations (Jonot, Villes, 1976, p. 25-38). Fn effet, nous avons constaté que le remplissage de certaines fosses était constitué partiellement de gravats semblables à ceux de la couche 8 dans laquelle elles ont été creusées. Toutefois, on peut très bien imaginer que ces fosses aient été utilisées dans un état proche de celui que nous avons pu observer, c'est-àdire dotées d'une large ouverture et peu profondes. Nous trouvons des exemples assez semblables dans le

30 Ce qui est par exemple le cas des silos du Castellas de Cucuron (Fixot, 1980).

31 Sur un total de vingt-huit fosses, six ont seulement ètè aperçues dans la berme et vingt relevées en plan, cinq présentent un diamètre inférieur a $1 \mathrm{~m}(2,14,16,17$ et 20), les autres diametres varient entre $1,06 \mathrm{~m}$ et $1,48 \mathrm{~m}$. Nous connaissons la profondeur de huit fosses $(1,3,4,5,6,7,9$ et 15$)$ elle est. comprise entre $0.64 \mathrm{~m}$ et $1,03 \mathrm{~m}$; elle est toujours inférieure au diamètre, ce qui diffère de ce que l'on a coutume de rencontrer.

32 A Aix-en-Provence, par exemple, sur le site de l'Archevèché, la capacité des silos est de l'ordre de 1 à $2 \mathrm{~m}^{3}$ (Fixot et alii, 1986, p. 243). Des silos avaient èté découverts à Fréjus lors de fouilles effectuées en 1955 (Février, 1956a, p. 46).
Nord de la France pour la période protohistorique (Jonot, Villes, 1981). Dans ce cas, les conditions d'étanchéité et d'isothermie nécessaires à la conservation des grains sur une longue période ne peuvent pas être obtenues. Toutefois, ce type d'excavation peut servir à stocker toute autre denrée (tubercules, fruits secs...) et ceci pendant unc période plus ou moins longue. Les parois des silos ne comportaient aucune trace de traitement particulier ou d'un revètement quelconque mais il est possible d'envisager, en raison de leur forme cylindrique et de leur faible profondeur, l'utilisation d'un contenant (tonneau, par exemple).

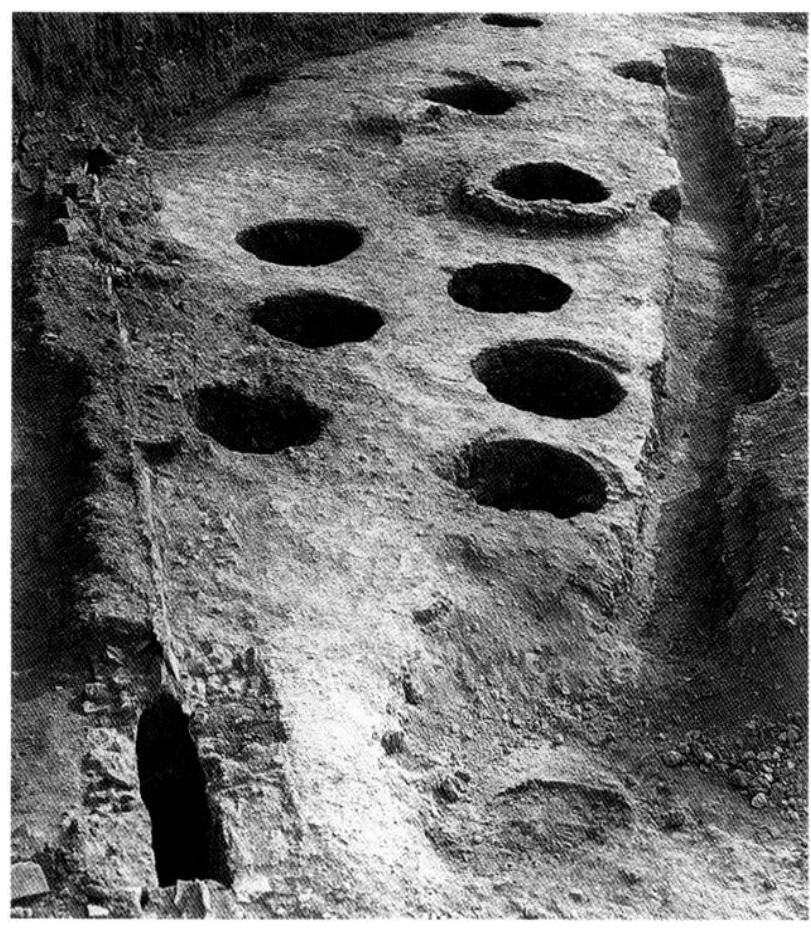

Fig. 54 - Vue des alignements des silos de l'état V.
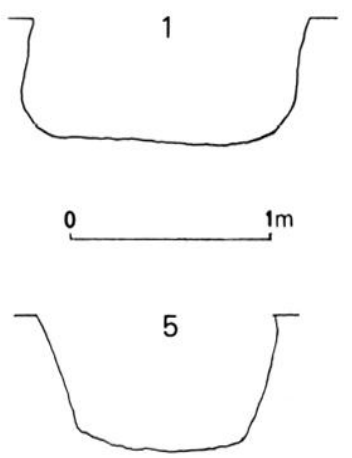
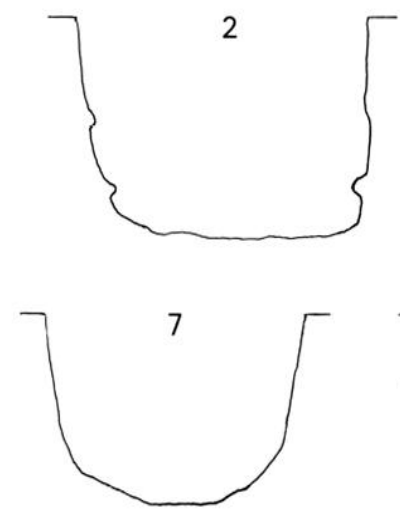
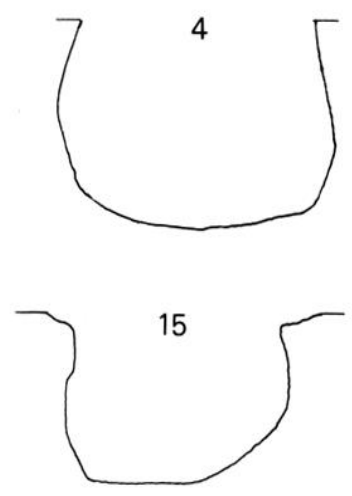

Fig. 55 - Profils des silos. 

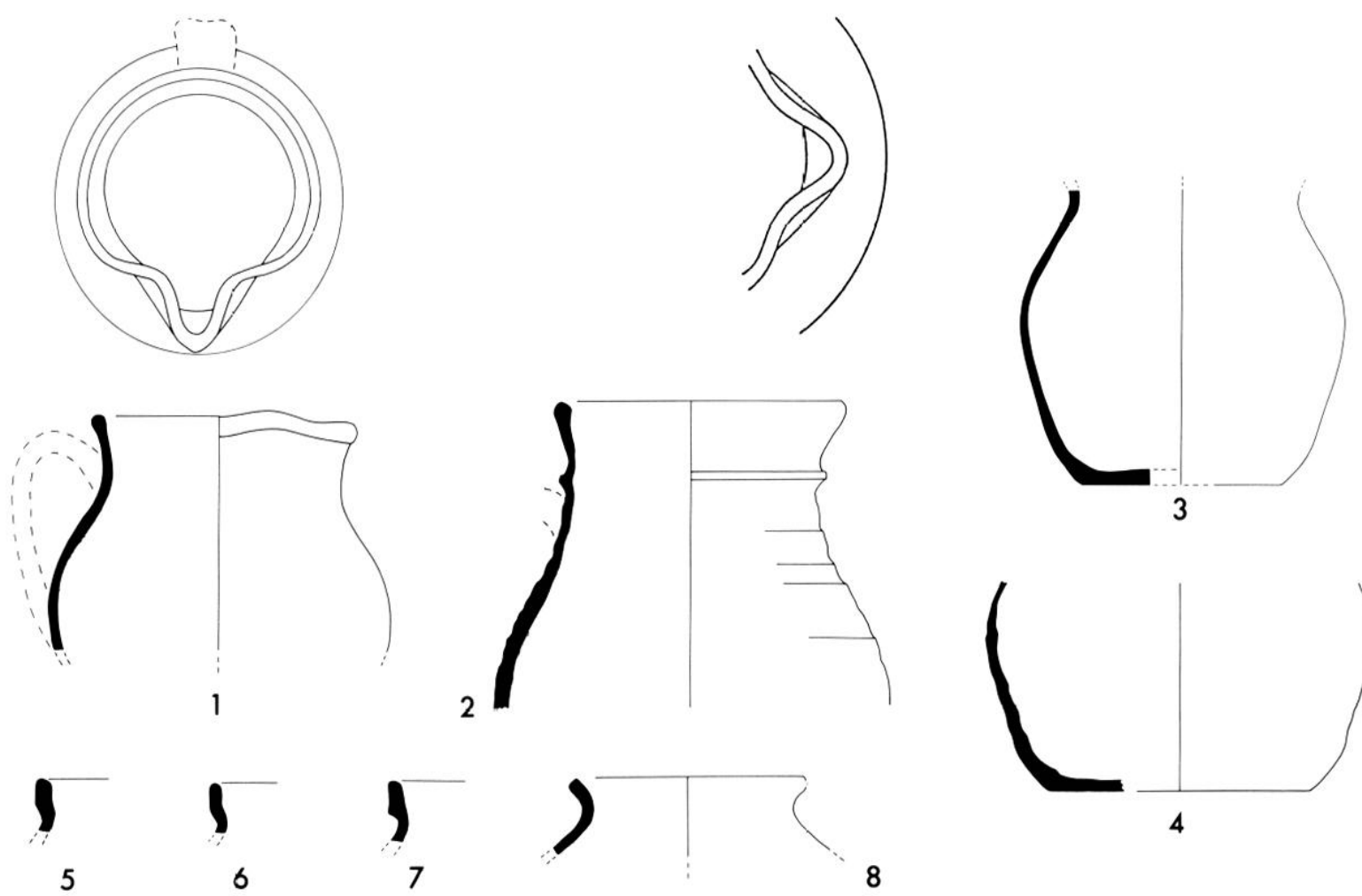

6
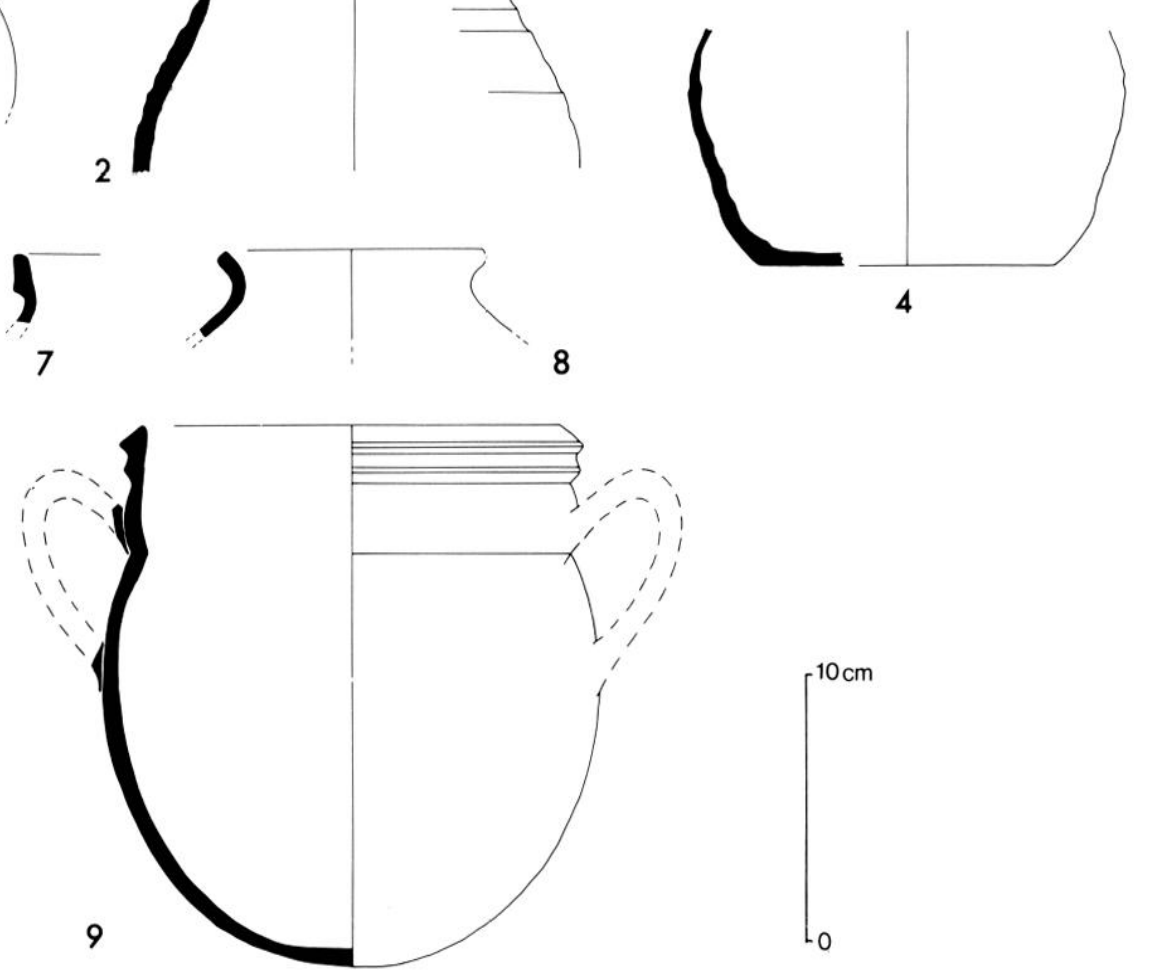

10
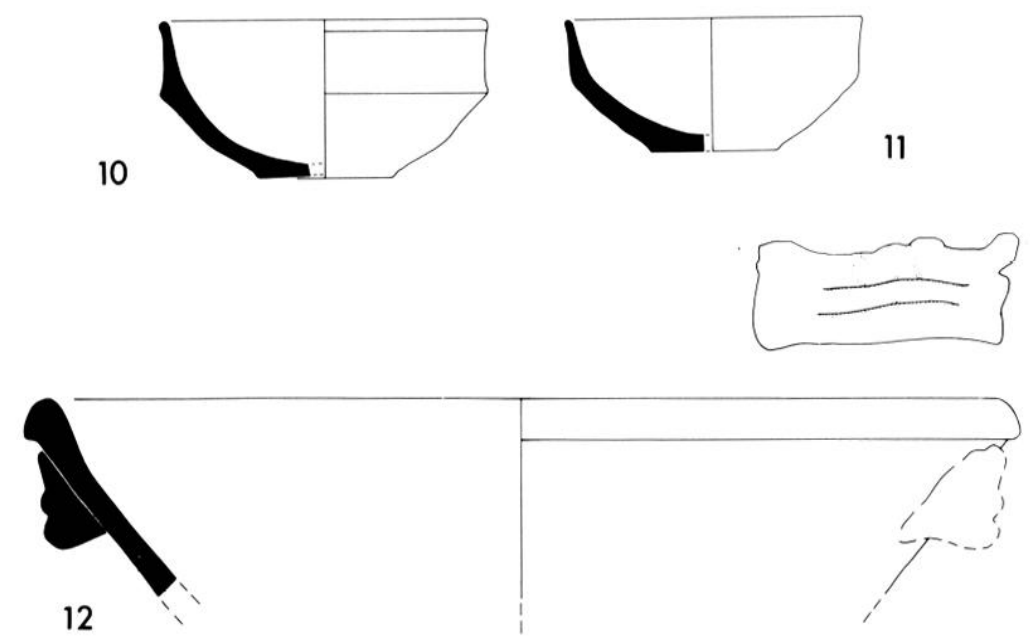

Fìg. 56 - Céramiques culinaires d'époque médièvale : pégaus ( $\left.n^{\circ s} 1-8\right)$; marmite $\left(n^{\circ} 9\right)$; céramiques d'époque moderne ( $\left.n^{\circ s} 10-12\right)$. 


\section{LE REMPLISSAGE DES SILOS}

Après leur abandon certaines fosses se sont semble-t-il effondrées, leur comblement est alors partiellement constitué de gravats de construction et de matériel résiduel $(8,9,11)$. D'autres, absolument stériles, ont peut-être bénéficié d'apport de matériaux permettant ainsi leur remplissage rapide $(2,16,17)$. Les deux opérations pouvant d'ailleurs être concomitantes comme dans la fosse 3 dont la partie supérieure a été comblée de cailloux.

Huit fosses, utilisées comme dépotoir, sont comblées de terre noire, d'écailles et d'arêtes de poissons, d'os animaux et de charbons de bois provenant de vidanges de foyers. Les céramiques issues de ces silos appartiennent à la catégorie des céramiques grises réalisées en cuisson réductrice. Six fosses ont livré une soixantaine de tessons de céramiques grises appartenant à des formes fermées (Faure-Boucharlat et alii, 1978; Fixot, Pelleticr, 1983). Il s'agit de vases à fond plat ou légèrement bombé, dont la liaison avec la panse est bien marquée (fig. $56, \mathrm{n}^{\mathrm{os}} 3$ et 4 ). Les bords sont évasés (fig. $56, n^{\circ} 8$ ) ou en bandeau (fig. $\left.56, n^{\circ s} 5-7\right)$, les formes sont trapues et caractéristiques des $\mathrm{XI}^{\mathrm{e}}$ et $\mathrm{XII}^{\mathrm{e}} \mathrm{s}$. (voire du $\mathrm{XIII}^{\mathrm{e}} \mathrm{s}$.). Deux de ces vases sont dotés d'un bec verseur et certainement d'une anse (fig. $56, n^{\circ} 1$ et 2 ). Aucun des tessons étudiés n'a livré de traces de décor.

En relation avec ce niveau, nous avons également mis au jour une structure de forme quadrangulaire (St 20) partiellement détruite au sud et à l'ouest (fig. 53). Elle est constituée d'un béton, dotée d'un puisard en son centre et limitée à l'est et au nord par un muret de pierres assemblées au mortier. Large de $0,32 \mathrm{~m}$, il est conservé sur une hauteur de $0,54 \mathrm{~m}$. Les proportions exactes de cette construction dont les dimensions minimales sont de $1,50 \mathrm{~m}$ sur $1,65 \mathrm{~m}$ sont difficiles à restituer; on peut penser qu'il s'agissait d'une cuve ou d'un bassin, peut-être lié à une activité artisanale?

Cette aire de stockage est la dernière activité qu'ait connue le site, son installation dans cette partie de la ville n'était probablement pas le fait du hasard puisque nous sommes à proximité du port qui à cette époque communiquait encore avec la mer (Aubenas, 1881, p. 588). Les denrées pouvaient séjourner sur ce terre-plein avant leur embarquement sur un navire ou leur départ pour l'intérieur des terres. Il est difficile de préciser la durée de vie de telles structures mais le comblement des silos ne contient que de la céramique grise ce qui les place entre le $\mathrm{XI}^{\mathrm{e}}$ et le $\mathrm{XII}^{\mathrm{e}} \mathrm{s}$. (voire le XIII's.). Toutefois, tous les silos n'ont pas fourni de matériel et ils n'ont pas pu, pour des raisons de délai de fouille, être livrés à nos investigations. Il nous est par conséquent impossible de savoir si certaines fosses ne sont pas postérieures à celles qui ont pu être datées.

\section{**}

L'ultime fait marquant dans l'évolution de ce site est sans aucun doute la période d'activité de l'aire de stockage. Un autre élément se rattachant à l'époque médiévale, la tombe 1, constituée d'un coffrage de pierre, est située dans la partie ouest du terrain (fig. 39); par ailleurs, les couches de terre arable contenaient, entre autres, une marmite de forme globulaire, à pâte rouge, présentant à l'intérieur des traces de vernis plombifère, datée des $x_{11}{ }^{\circ}$ ou XIVe s. (fig. 56, no 9) (Démians d'Archimbaud, 1980 , p. 327-335).

Les vestiges extrêmement ténus des niveaux postérieurs semblent uniquement liés à une activité de jardin dont l'aménagement se manifeste par la création d'une citerne et de quatre puits. L'un d'eux était partiellement comblé de céramiques vernissées d'époque moderne: bols (fig. $56, \mathrm{n}^{\mathrm{os}} 10$ et 11), écuelles, vases à liquide, jattes (fig. $56, n^{\circ} 12$ ), provenant pour la plupart des nombreux ateliers de Fréjus (Amouric, Landuré, 1985).

Pour l'époque moderne, nous savons que ce secteur de la ville était occupé par des zones de culture $^{33}$. Cette vocation, peut-être plus ancienne, s'est longtemps maintenue puisque le cadastre de 1825 révèle un terrain encore libre de toute construction à l'exception d'un hangar situé dans la partie ouest de cette parcelle, qui sera d'ailleurs préservée jusqu'en 1986. Les terrasses de ce terrain perpétuaient ce que les remblais installés au ${ }^{\prime I^{\circ}} \mathrm{s}$. avaient déterminé et le mur de limite cadastrale est reposait directement sur le mòle antique (M18). Ainsi, longtemps épargné par l'urbanisation, ce secteur de la ville avait gardé dans son parcellaire les traces des structures gallo-romaines, marquant une continuité depuis l'Antiquité jusqu'à nos jours.

\section{Isabelle BÉraUd \\ Chérine Gérara \\ Corinne Landuré}

33 Comme l'illustre un plan de 1634, manuscrit B.N. (GED 14685). 
Tableau I - Céramiques de l'état I.

\begin{tabular}{|c|c|c|c|c|c|c|c|c|c|c|c|c|c|c|}
\hline \multirow[b]{2}{*}{ Couches } & \multicolumn{7}{|c|}{ Sondage E } & \multicolumn{5}{|c|}{ Sondage $\mathrm{F}$} & \multicolumn{2}{|c|}{ Pièce 3} \\
\hline & 10 & 11 & 12 & 13 & 14 & 15 & 16 & 9 & 10 & 11 & 12 & 13 & 11 & 13 \\
\hline Sigillée sud-gauloise & & 3 & 2 & 2 & 2 & 4 & 13 & & 2 & 9 & & & 11 & 7 \\
\hline Arétine & 1 & & 1 & & & & & & & 2 & & & 1 & 11 \\
\hline Paroi fine & 1 & 1 & & 2 & 2 & & 5 & & 6 & 85 & & & 1 & 19 \\
\hline Grise de Vaison & & & & & 5 & 5 & 41 & 6 & 4 & 162 & & & 18 & 48 \\
\hline Rouge pompéien & & & & & & & & & & & & & 2 & 3 \\
\hline Lampes & & & 1 & 1 & & & 2 & & 2 & 40 & & 1 & 3 & 18 \\
\hline Commune engobée & & & & & & & & & & 51 & & & 13 & 7 \\
\hline Commune grise & & 3 & & 1 & & & & & & & & & 6 & 28 \\
\hline Commune brune & 1 & & 1 & & 3 & 2 & 17 & 5 & 2 & 136 & & 1 & 10 & 36 \\
\hline Commune claire & 9 & 11 & 17 & 10 & 9 & 15 & 24 & 9 & 20 & 245 & & 4 & 29 & 211 \\
\hline Amphores & 5 & 3 & 20 & 4 & 13 & 16 & 121 & 33 & 26 & 559 & 17 & 21 & 27 & \\
\hline Autres & & & & & & & & & & 1 & & & & \\
\hline Total & 17 & 21 & 42 & 20 & 34 & 42 & 223 & 53 & 62 & 1290 & 17 & 27 & 121 & 388 \\
\hline
\end{tabular}


Tableau II - Cèramiques de l'ètat II.

\begin{tabular}{|c|c|c|c|c|c|}
\hline & $\mathrm{P} 5$ & P7 & P13 & \multicolumn{2}{|c|}{ P3 } \\
\hline & C 10 & C $5-6$ & C 6 & C 9 & C. 10 \\
\hline \multicolumn{6}{|l|}{ DSP } \\
\hline \multicolumn{6}{|l|}{ Claire D } \\
\hline \multicolumn{6}{|l|}{ Claire B } \\
\hline \multicolumn{6}{|l|}{ Claire A } \\
\hline \multicolumn{6}{|l|}{ Culinaire africaine } \\
\hline Sigillée sud-gauloise & 64 & 9 & 3 & 8 & 1 \\
\hline Arétine & 6 & 2 & & & \\
\hline Paroi fine & 18 & 1 & & 3 & 2 \\
\hline Grise de Vaison & 34 & 2 & 3 & 15 & 1 \\
\hline Rouge pompéien & 3 & 1 & & & \\
\hline Lampes & 5 & & & & 1 \\
\hline Commune engobée & 4 & 2 & 2 & 5 & \\
\hline Commune grise & & 1 & & & 4 \\
\hline Commune brune & 12 & & & 2 & 2 \\
\hline Céramique modelée & 52 & 4 & & & \\
\hline Commune claire & 52 & 15 & & 76 & 14 \\
\hline Amphores & 415 & 47 & 3 & 22 & 23 \\
\hline Autres & 7 & & & & \\
\hline Total & 705 & 81 & 11 & 131 & 48 \\
\hline
\end{tabular}


Tableau III - Céramiques de l'état III.

\begin{tabular}{|c|c|c|c|c|c|c|c|c|c|c|c|c|c|c|}
\hline & \multicolumn{11}{|c|}{ État IIIA } & \multirow{2}{*}{\multicolumn{3}{|c|}{ État IIIB }} \\
\hline & \multicolumn{5}{|c|}{ P3 } & \multicolumn{3}{|c|}{ P5 } & \multicolumn{3}{|c|}{ P7 } & & & \\
\hline & C5 & C6 & C7 & $\mathrm{C} 8$ & M38 & C7 & $\mathrm{C} 8$ & $\mathrm{C} 9$ & C2 & C3 & $\mathrm{C} 4$ & $\begin{array}{l}\text { M21 } \\
\text { ext. }\end{array}$ & $\begin{array}{l}\text { M21 } \\
\text { int. }\end{array}$ & C8 \\
\hline DSP & & & & & & 1 & & & & & & & & \\
\hline Claire D & & & & & & & & & & & 2 & & & \\
\hline Claire B & & & & & 3 & 1 & & & & & & & & \\
\hline Claire A & & & & & 1 & 1 & 1 & & 8 & 27 & 8 & & 2 & \\
\hline Culinaire africaine & & & & 1 & & & & & & & 21 & 2 & & \\
\hline Sigillée sud-gauloise & 7 & 4 & 3 & & 7 & 42 & 86 & 21 & 148 & 17 & 28 & 9 & 3 & 2 \\
\hline Arétine & & & & & 2 & & 16 & 5 & 4 & & 5 & & & 2 \\
\hline Paroi fine & 27 & 4 & & 2 & & 25 & 50 & 7 & 60 & 11 & 12 & & & \\
\hline Grise de Vaison & & 8 & 3 & & & 41 & 121 & 7 & 26 & 18 & 16 & 2 & & 1 \\
\hline Rouge pompéien & & & & & & 5 & 8 & 2 & 8 & 3 & & & & \\
\hline Lampes & 4 & 1 & 1 & & 1 & 7 & 20 & 2 & 24 & 7 & 15 & & 1 & \\
\hline Commune engobée & 9 & 1 & 1 & & 1 & 25 & 80 & 1 & 106 & 24 & 26 & & 2 & \\
\hline Commune grise & 27 & & & 10 & & 1 & 25 & 1 & 18 & & 1 & 2 & 2 & 1 \\
\hline Commune brune & 15 & 9 & & 5 & 4 & 79 & 208 & 2 & 48 & 19 & 1 & 1 & & 1 \\
\hline Céramique modelée & & & & & & & & 4 & 86 & 35 & 22 & 1 & & \\
\hline Commune claire & 123 & 27 & 24 & 34 & 10 & 52 & 136 & 8 & 176 & 38 & 35 & 1 & 2 & 21 \\
\hline Amphores & 99 & 34 & 15 & 39 & 136 & 42 & 906 & 93 & 2158 & 281 & 25 & 17 & 6 & 58 \\
\hline Autres & & & & 1 & & 2 & & 1 & 58 & 15 & 2 & 3 & & \\
\hline Total & 311 & 88 & 47 & 92 & 165 & 730 & 1593 & 154 & 2819 & 428 & 409 & 38 & 18 & 86 \\
\hline
\end{tabular}


Tableau IV - Céramiques de l'ètat IVA.

\begin{tabular}{|c|c|c|c|c|c|c|c|}
\hline & \multicolumn{2}{|c|}{ P4 } & \multicolumn{5}{|c|}{$\mathrm{P} 10$} \\
\hline & $\mathrm{S} 4$ & Foyer A & $\mathrm{C} 8$ & Foyer A & Foyer D & C9 & C10 \\
\hline DSP & & 2 & 12 & & & 8 & \\
\hline \multicolumn{8}{|l|}{ Late Roman $C$} \\
\hline Claire D & & 28 & 136 & 1 & 4 & 72 & 15 \\
\hline Claire C & & & 3 & 2 & & 10 & 2 \\
\hline Luisante & & & 3 & & & 6 & \\
\hline Claire B & & & 6 & & & & \\
\hline Claire A & & & 9 & & & 1 & \\
\hline Culinaire africaine & & & 24 & 1 & & 19 & 3 \\
\hline Sigillée sud-gauloise & 1 & & 14 & 1 & 1 & 17 & 7 \\
\hline Arétine & & & 11 & & & & 1 \\
\hline Paroi fine & & & & & & 5 & 1 \\
\hline Grise de Vaison & & & & & & 4 & 5 \\
\hline Lampes & 1 & & 5 & & & & \\
\hline Commune engobée & & 3 & 19 & & & 10 & 7 \\
\hline Commune grise & 1 & 3 & 3 & & 5 & 4 & \\
\hline Commune brune & & 4 & 185 & 3 & 1 & 100 & 22 \\
\hline Commune claire & 2 & 3 & 91 & 5 & 7 & 104 & 21 \\
\hline Céramique modelée & & & 21 & & & 2 & \\
\hline Amphores & 1 & 13 & 1362 & 24 & 25 & 859 & 156 \\
\hline Total & 6 & 56 & 1904 & 37 & 43 & 1221 & 240 \\
\hline
\end{tabular}


Tableau V - Céramiques de l'ètat IVB.

\begin{tabular}{|c|c|c|c|c|c|c|c|c|c|c|c|c|}
\hline & \multicolumn{9}{|c|}{ P5 } & \multicolumn{3}{|c|}{ P4 } \\
\hline & C3a & $\mathrm{C} 3 \mathrm{~b}$ & $\mathrm{C} 4$ & Foyer A & $\mathrm{C} 5$ & $\mathrm{~F} 1$ & Foyer B & $\mathrm{C} 6 \mathrm{~b}$ & $\mathrm{C} 6 \mathrm{c}$ & $\mathrm{FC}$ & FCa & $\mathrm{FCb}$ \\
\hline DSP & & & 1 & & & & & 2 & 5 & & & \\
\hline Late Roman $C$ & & & 1 & & & & & & & & & \\
\hline Claire D & & 1 & & & 4 & 3 & & & & 4 & 1 & 3 \\
\hline Claire C & & & & & & & & & & & & \\
\hline Luisante & & & & & & & & & & & & \\
\hline Claire B & & & & & & & & 2 & & 1 & & \\
\hline Claire A & & & & & 4 & & & 8 & & & & \\
\hline Culinaire africaine & & & 1 & & & 1 & & & & & & 1 \\
\hline Sigillée sud-gauloise & 1 & 2 & 20 & & 68 & 9 & & 22 & 24 & & & 3 \\
\hline Arétine & & & & & 14 & & & & 6 & & & \\
\hline Campanienne & & & & & & 1 & & & & & & \\
\hline Paroi fine & & 1 & & & 24 & 4 & & 9 & 19 & & 3 & 1 \\
\hline Grise de Vaison & & 1 & 12 & 4 & 51 & 5 & 22 & 28 & 25 & & & \\
\hline Rouge pompéien & & & 1 & & & & & & & & & \\
\hline Lampes & & & 4 & & 20 & 2 & & 2 & 1 & & & \\
\hline Commune engobée & & & & 2 & 7 & 7 & & 9 & 6 & & & 2 \\
\hline Commune grise & 3 & & 4 & 8 & 26 & 15 & & 12 & & 16 & 1 & \\
\hline Commune brune & & & 5 & 1 & 26 & 12 & 10 & & 1 & & & 15 \\
\hline Commune claire & 3 & 4 & 22 & 3 & 45 & 13 & 9 & 26 & 16 & 11 & 15 & 14 \\
\hline Céramique modelée & & & 7 & & 48 & & & 18 & 35 & & & \\
\hline Amphores & & 1 & 34 & 33 & 386 & 39 & 13 & 158 & 144 & 12 & 2 & 34 \\
\hline Total & 7 & 10 & 112 & 51 & 723 & 111 & 54 & 296 & 282 & 44 & 22 & 73 \\
\hline
\end{tabular}


Tableau VI Céramiques de l'état IVC:

\begin{tabular}{|c|c|c|c|c|}
\hline & M24 & 29 & 30 & M35 \\
\hline DSP & 5 & & 3 & 1 \\
\hline Late Roman $C$ & & 1 & & \\
\hline Claire D & 15 & 2 & 4 & \\
\hline \multicolumn{5}{|l|}{ Claire $\mathrm{C}$} \\
\hline \multicolumn{5}{|l|}{ Luisante } \\
\hline Claire B & 1 & & 1 & \\
\hline Claire A & 2 & & & \\
\hline Culinaire africaine & & 1 & 1 & 1 \\
\hline Sigillée sud-gauloise & 14 & 6 & & \\
\hline \multicolumn{5}{|l|}{ Arétine } \\
\hline \multicolumn{5}{|l|}{ Campanienne } \\
\hline Paroi fine & 2 & & 2 & 1 \\
\hline Grise de Vaison & 4 & & & \\
\hline \multicolumn{5}{|l|}{ Rouge pompéien } \\
\hline Lampes & 2 & & & \\
\hline Commune engobée & 4 & & & 1 \\
\hline Commune grise & 17 & & 1 & \\
\hline Commune brune & 22 & 94 & 4 & \\
\hline Commune claire & 73 & 65 & 26 & 1 \\
\hline Céramique modelée & 3 & & & 1 \\
\hline Amphores & 204 & 93 & & 5 \\
\hline Total & 368 & 262 & 78 & 11 \\
\hline
\end{tabular}

Tableatu VII -- sigillés claires D).

\begin{tabular}{|l|c|c|c|c|c|c|c|c|c|}
\hline & P4 & P10 & P5 & P5 & P5 & P5 & P4 & Tr. & M24 \\
& F3 & C3 & S4 & C5 & FC & 30 & \\
\hline Hayes 58 & 1 & 2 & & & & & & & \\
\hline Hayes 59 & 3 & 8 & & & & & & & \\
\hline Hayes 61B & 3 & 18 & & 1 & & 1 & & & \\
\hline Hayes 67 & & 12 & & & & & & & \\
\hline Hayes 68 & & 1 & & & & & & & \\
\hline Hayes 69 & & 3 & & & & & & & \\
\hline Hayes 73 & & 1 & & & & & & & \\
\hline Hayes 87C & & & & & & & & & 2 \\
\hline Hayes 88 & & & & & & & 2 & & 1 \\
\hline Hayes 91AB & & 14 & & & & & & & \\
\hline Hayes 99 & & 1 & 1 & & 1 & 1 & & 1 & 1 \\
\hline Total & 7 & 60 & 1 & 1 & 1 & 2 & 2 & 1 & 4 \\
\hline
\end{tabular}




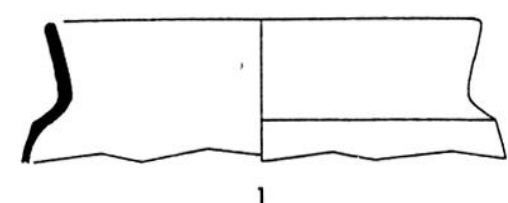

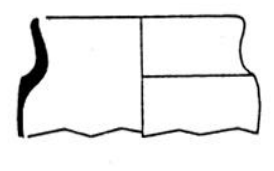

2
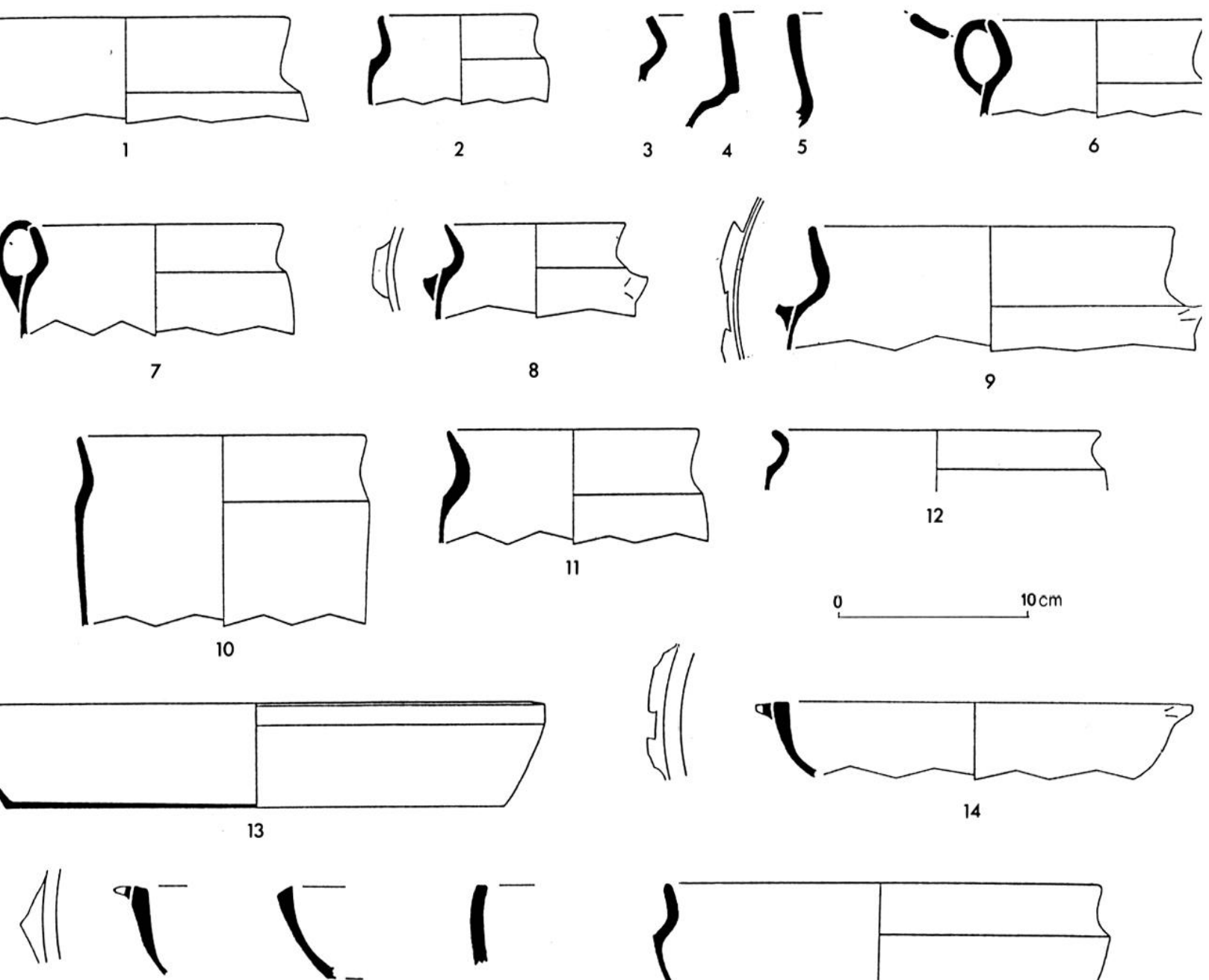

15
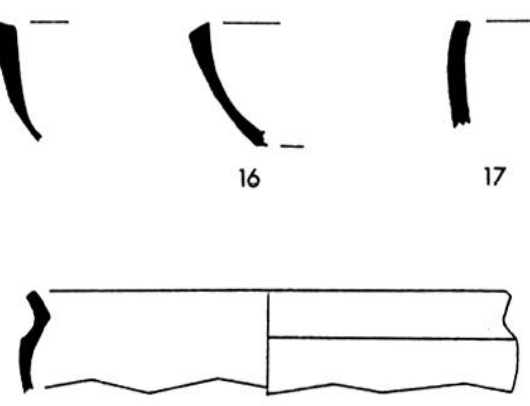

19

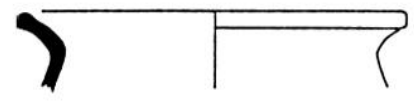

22
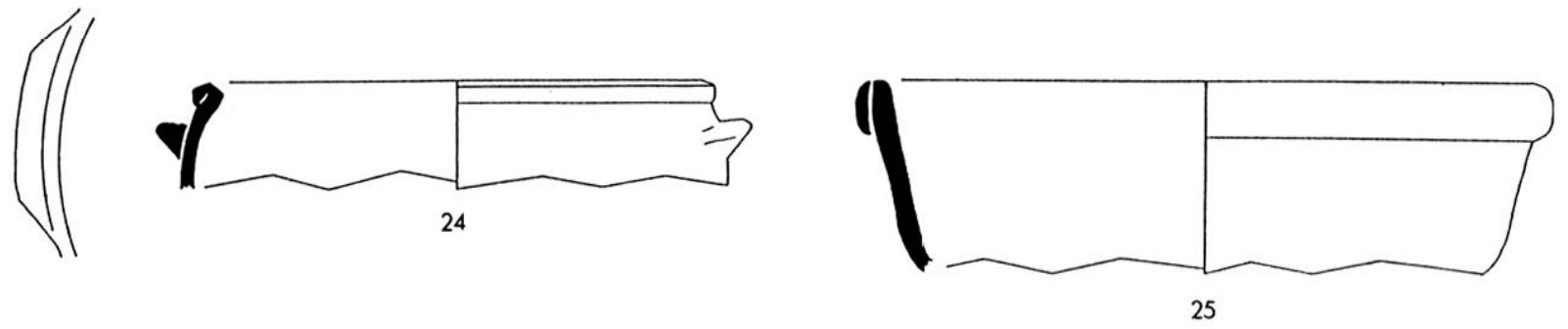

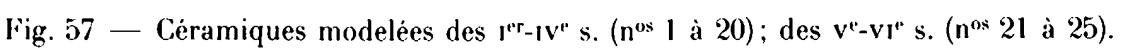




\title{
ANNEXE \\ La céramique modelée de la Porte d'Orée à Fréjus \\ (Var)
}

\author{
par Jacques BÉRATO*
}

Le matériel issu des fouilles de la Porte d'Orée correspond à une longue période d'occupation qui permet de suivre l'évolution de la céramique modelée du $\mathrm{I}^{\mathrm{er}}$ au $\mathrm{IV}^{\mathrm{e}} \mathrm{s}$. et d'en préciser certains points chronologiques.

Nous envisagerons successivement la céramique modelée des $\mathrm{I}^{\mathrm{er}}$ et $\mathrm{IV}^{\mathrm{e}} \mathrm{s}$, puis des $\mathrm{v}^{\mathrm{e}}$ et $\mathrm{vI}^{\mathrm{e}} \mathrm{s}$.

\section{LA CÉRAMIQUE MODELÉE DES er-IV $^{\mathrm{e}} \mathrm{s}$.}

\section{MODALITÉS DE FABRICATION}

Très résistante au feu, elle est toujours réalisée à l'aide d'une argile non calcaire, à laquelle est mêlé un dégraissant de mica bien visible à l'œil nu (diamètre : 0,05 à $1 \mathrm{~mm}$, parfois paillette de $5 \mathrm{~mm}$ ) et de quartz (diamètre : 0,3 à $0,5 \mathrm{~mm}$, rarement 1 à $3 \mathrm{~mm}$ ). La couleur de la pâte varie du brun clair au brun noir, ce qui s'cxplique par une cuisson réductrice, avec phénomène de réoxydation en post-cuisson. Les techniques de finition font toujours appel au lissage, pour la face externe du bord ou de l'encolure et l'intérieur du récipient, et au grattage du flanc et $\mathrm{du}$ fond. Cette technique est caractéristique de cette production. Le démaigrissement permet d'obtenir des parois plus fines, un récipient plus léger et une surface résistant mieux aux chocs thermiques et diffusant mieux la chaleur.

\section{ÉTUDE DESCRIPTIVE}

219 bords ont été décomptés.

\section{Urnes}

Elles sont représentées par 144 individus, soit $66 \%$ de l'ensemble de la céramique modelée.

En fonction de l'encolure on peut distinguer deux types.

Type $a$ : encolure au profil caractéristique en baïonnette (127 individus, fig. $57, n^{\text {os }} 1$ à 9$)$ : haut col droit et évasé, épaule courte légèrement ascendante. Carène vive. Corps tronconique, parfois renflé dans son tiers supérieur, à paroi le plus souvent très fine $(0,3-0,4 \mathrm{~mm})$. La surface d'appui est plate. Sept individus $(6 \%$ des urnes) portent un moyen de préhension : anse rubanée s'insérant sur la lèvre et la carène (4 individus, fig. 57, nos 6 et 7 ); tenon aplati collé sur la carène, simple ( 2 individus, fig. $\left.57, n^{\circ} 8\right)$ ou double (1 individu, fig. 57, $\mathrm{n}^{0} 9$ ).

Type $b$ : encolure affadie au profil en baïnnette (17 individus, fig. 57, $\mathrm{n}^{\mathrm{os}} 10$ à 12). La longueur de l'épaule est identique à celle du col. La jonction colépaule est souvent épaissie. La carène vive peut être émoussée. Le corps tronconique, parfois renflé dans son tiers supérieur, a des parois plus épaisses que celles de la forme précédente. La surface d'appui est plate. Il n'y a pas dans cette série de moyen de préhension. Le diamètre externe à l'ouverture des urnes varie de 8 à $30 \mathrm{~cm}$, avec un regroupement entre 12 et $20 \mathrm{~cm}$ et une valeur préférentielle de $16 / 18 \mathrm{~cm}$, sans variation statistique entre les deux types.

\section{Jattes}

Elles sont représentées par 66 individus, soit $30 \%$ de la céramique modelée (fig. 57 , nos 13 à 17).

Le diamètre externe à l'ouverture varie de 16 à $38 \mathrm{~cm}$ avec une valeur préférentielle de $24 / 26 \mathrm{~cm}$. Récipient ouvert, peu profond, aux parois évasées, galbées ou droites, avec rebord redressé ou légèrement rentrant. La morphologie de la lèvre aplatie, arrondie, en biseau interne ou externe, est liée aux variations du façonnage et sans valeur typologique. La surface d'appui est plate. Quatorze récipients (21\% des jattes) possèdent des éléments de préhension, le plus souvent fixés sur le haut du bord, contre la lèvre, ils sont en forme de lunule simple (non dessi-

\footnotetext{
* Centre archéologique du Var, Établissement de Toulon, 14, boulevard Bazeilles, 83000 Touron.
} 
nés mais identiques à celui de la fig. 57, $\left.n^{\circ} 8\right)$ ou de double tenon (fig. $57, n^{\circ s} 14$ et 15 ).

\section{Faitouts}

Ils sont représentés par 6 individus, soit $3 \%$ de la céramique modelée (fig. $57, n^{\text {os }} 18$ et 19 ).

Le diamètre varie de 20 à $26 \mathrm{~cm}$. Ce récipient ouvert dont le diamètre externe à l'ouverture est. compris entre 2 et 4 fois la hauteur, a pu ètre confondu avec une marmite qui est plus profonde. L'encolure a un profil en baïonnette. La jonction colépaule peut être épaissie et marquée à sa face interne par une arête. I e flanc est évasé. I a surface d'appui peut être plate ou convexe. Trois individus sont porteurs d'un moyen de préhension : lunule aplatie (1 individu) ou en double tenon ( 2 individus).

\section{Couvercles}

Ils sont représentés par 3 individus, soit $1 \%$ de la céramique modelée.

Type discoïdal, 2 individus, diamètre : $22 \mathrm{~cm}$ (non dessiné car trop fragmentaire).

Type trapézoïdal, 1 individu, diamètre: $34 \mathrm{~cm}$ (fig. $\left.57, n^{\circ} 20\right)$.

\section{CONSIDÉRATIONS GÉNÉRALES, DIFFUSION ET CHRONOLOGIE}

Les différentes formes de la céramique modelée de la période I"r-IV" s. constituent les èléments d'une batterie de cuisine. (Ce fait explique que les surfaces des récipients soient souvent couleur brun noir foncé, fuligineuses à l'extérieur et carbonnées à l'intérieur avec des dépôts alimentaires qui s'écaillent.

La jatte peut servir à la cuisson du pain et à la préparation de ragoùt ou de friture, les urnes à l'ébullition de l'eau ou à la cuisson par immersion des aliments; le faitout à la cuisson des aliments, particulierement à l'étouffée.

L'histogramme de répartition des diamètres à l'ouverture caractérise bien les différentes formes de céramique et cette utilisation culinaire (fig. 58): urne haute et étroite, jatte large et profonde, marmite large, mais plus profonde que la jatte.

L'usage est donc principalement restreint au domaine culinaire et au stockage alimentaire, accessoirement aux dépòts funéraires.

Ia répartition entre les différentes formes est pratiquement identique à celle de Rivet (1982), pour le Clos de la Tour et un ensemble de sites de Frejus : urne $69 \%$, jatte $29 \%$, faitout $3 \%$.

I a prédominance des urnes sur les jattes et sur-

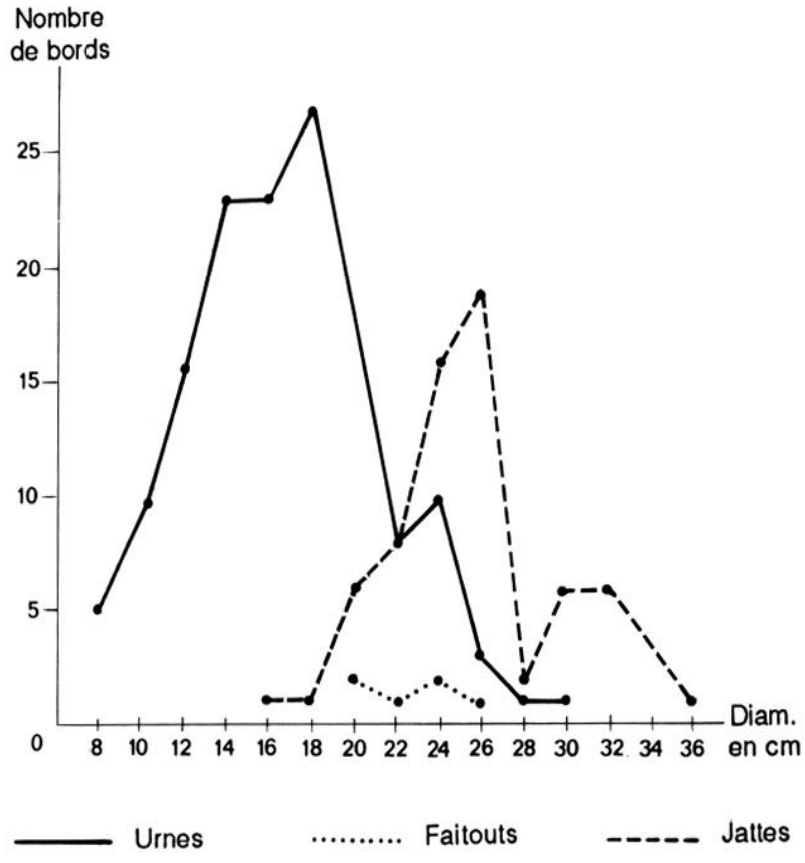

Fig. 58 - Ilistogramme des diamètres des récipients.

tout des faitouts est retrouvée sur tous les sites varois.

I. a proportion de céramique modelée par rapport à l'ensemble de la céramique oscille autour de $5 \%$ et rejoint le chiffre trouvé par Kivet (1982). Ce pourcentage de céramique modelée apparaît plus important sur les sites ruraux de l'intérieur du Var.

Les composants non plastiques de la pâte de cette céramique modelée nous incitent à situer les ateliers de production dans le massif ancien des Maures ou à sa périphérie dans la vallée de l'Argens. Nous ne connaissons toutefois aucune localisation de four et nous n'avons jamais trouvé de raté de cuisson.

La présence de céramique modelée à bord des épaves des Roches d'Aurelles et du Dramont G en association avec de la céramique tournec provenant d'ateliers fréjusiens atteste son origine varoise, et que parallèlement à la diffusion terrestre dans tout le Var, ce produit a été l'objet d'un commerce maritime par cabotage côtier. On le retrouve sur la côte ligure de la limite orientale des Bouches-du-Rhône jusqu’à Vintimille (Bérato et alii, 1986a).

Les jattes avec moyen de préhension sont retrouvées sur les sites côtiers et urbains : Fréjus, TouIon-Ferrailleur $(12 \%)$, Olbia $(7 \%)$, Saint-Cyr-lesLecques. Elles sont absentes sur les sites ruraux et de 
l'intérieur du Var: Saint--Michel à La Carde, Le Grand Loou à La Roquebrussanne, La Meynade-Le Clafard à Six-Fours (Bérato, 1982), L'Ormeau à Taradeau (Bérato, 1984). Ce caractère typologique représente-t-il un faciès côtier, lié au commerce maritime, ou est-il le fait d'un aléa de fouilles?

A l'exclusion de l'affadissement de l'encolure en baïonnelte des urnes, avec prédominance du type b à partir du $\mathrm{II}^{\mathrm{e}}$ s., surtout sur les sites ruraux, le faciès typologique est figé durant toute la période $\mathrm{I}^{\mathrm{er}}-\mathrm{IV} \mathbf{s}$. La chronologie a été précisée par des travaux préliminaires (Rivet, 1982; Bérato, 1981; Bérato el alii, 1986b).

L'apparition de cette céramique remonte au règne de Tibère soit $20 / 30$ de notre ère. A la Porte d'Orée, différentes formes sont présentes dès l'état I (milieu du $\mathrm{I}^{\mathrm{er}} \mathrm{s}$. de notre ère), datation qui ne nous permet pas de préciser l'existence d'un léger décalage chronologique retrouvé à l'Ormeau à Taradeau entre l'apparition des urnes de type a (deuxième quart du ${ }^{\text {er }} \mathrm{s}$.) et celle des urnes de type b (milieu du I $^{\text {er }}$ s.). Cette céramique modelée n'est plus retrouvée après le IV"s. (Bérato el alii, 1986b).

\section{CONCLUSIONS}

La céramique modelée à panse grattée des $\mathbf{I}^{e r}-\mathrm{IV}^{\mathrm{e}}$ s., qui est une production spécifique du Var, est toujours retrouvée lors des fouilles de sites varois, datés de cette période. Cette céramique s'exporte vraisemblablement à partir du port de Fréjus comme complément de fret, dans le cadre d'un commerce maritime par cabotage, sur la côte ligure entre Marseille et Vintimille. Le $v^{e} \mathrm{~s}$. marque la disparition de cette céramique avec grattage des flancs, au profit de nouveaux types.

\section{LA CÉRAMIQUE DES Ve-vie s.}

L'échantillon très réduit ( 6 bords) de céramique modelée de l'Antiquité tardive n'autorise qu'une etude descriptive des formes présentes et permet toutefois de préciser des chronologies déjà proposées.

\section{CÉRAMIQUE LOCALE}

Elle se caractérise par une pâte de couleur brun noir foncé, comportant du quartz et du mica. Le façonnage est grossier et le lissage des surfaces est irrégulier.

Deux types de pot se différencient selon la forme de l'encolure.
Type $a: 3$ individus (fig. 57, $\mathrm{n}^{\circ \mathrm{s}} 21$ et 22 ). Diamètre : 12 et $16 \mathrm{~cm}$ ( 2 individus). Le col curviligne court et évasé se continue avec l'épaule par une courbure régulière. Le corps est oblong.

Type $b: 1$ exemplaire (fig. 57, n" 23). Diametre : $20 \mathrm{~cm}$. Le col court est évasé, marqué à sa jonction avec l'épaule par une pliure externe nette et une arête à la face interne.

Parmi les quatre exemplaires de la Porte d'Orée qui viennent de l'état IV ( $\mathrm{V}^{\mathrm{r}}-\mathrm{vI}^{\mathrm{C}} \mathrm{s}$.) les pots de type a sont plutôt du $\mathrm{v}^{\mathrm{c}} \mathrm{s}$. Une céramique modelée semblable est attestée sur les sites varois datables du $\mathbf{V}^{\mathrm{C}}$-VII"s. : nécropole du Pauvadou à Fréjus, Olbia à Hyères, villa de Pardigon 2, La Ciroix-Valmer (Brun et alii, 1986) et au quartier Besagne à Toulon (Bérato et alii, 1986b).

I a similitude de forme des récipents et la proximité des sites indiquent qu'il s'agit probablement de productions locales varoises.

\section{LA CÉRAMIQUE MODELÉE D'IMPORTATION}

Jatte : 1 exemplaire (fig. 57, n²4).

Diamètre : $29 \mathrm{~cm}$. Un bandeau arrondi est collé sur le bord. I.e flanc est droit et évasé. La pâte comporte un dégraissant sableux (quartz). Flle est de couleur brun orangé à la face interne, et brun noir à la face externe avec dépôts fuligineux.

Cet exemplaire qui provient de l'état IV de la Porte d'Orée, daté du v $\mathrm{v}^{\mathbf{c}} \mathrm{s}$, est retrouvé à Carthage, Porto 'lorres (Sardaigne) et 'loulon (Bérato el alii, 1986b) dans des milieux du $\mathrm{v}^{\mathrm{e}} \mathrm{s}$.

Faitout : 1 exemplaire (fig. $\left.57, n^{\circ} 25\right)$ ).

Diamètre : $22 \mathrm{~cm}$. Le bord rentrant avec lèvre épaissie (par repliement de la paroi en dehors) est biseauté. Le flanc est galbé. Deux éléments de préhension en forme de lunule aplatie sont collés sur l'épaule. La pâte de couleur brun noir comporte un dégraissant de quartz et de calcaire en proportion moindre. Cet exemplaire, qui provient de l'état IV de la Porte d'Orée, daté du vi"s., est rencontré à Carthage, forme 20 de Fulford et Peacock (1984, p. 163 et 165 , fig. $58,20.1$ à 20.3), où elle est datée de 500 à $550 / 575$.

\section{CONCLUSIONS}

A partir du $v^{e}$ s., la céramique modelée est très minoritaire dans l'ensemble du mobilier en terre cuite. Une certaine incertitude règne en l'absence d'analyse de pâte, pour déterminer l'origine des récipients. Deux ensembles se différencient : 
- groupe d'origine locale au modelage irrégulier, que l'on rencontre à La Croix-Valmer (villa de Pardigon 2), Fréjus (nécropole du Pauvadou), Hyères (Olbia) et Toulon (quartier Besagne);

- groupe d'importation, au façonnage plus soigné, et où l'usage d'une tournette pour certaines finitions n'est pas à exclure.
Ces différentes formes appartiennent à un faciès céramologique méditerranéen qui se retrouve dans des ports : Carthage, Marseille, Porto Torres, Toulon, Vintimille. Elles attestent les liens qui existent, aux $v^{e}-v^{e}$ s., entre le Sud de la France, l'Afrique du Nord et la Méditerranée.

J.B.
Nota Bene

Équipe d'encadrement: I. Béraud, Ch. Gébara, Service archéologique municipal de Fréjus; R. Boiron, C. Landuré, archéologues contractuels.

Équipe de fouille : A. Conte, J. De Ridder, C. Lecat, D. Maiarelli.

Fouilleurs bénévoles: C. Boetti, F. Canovas, F. Cameron, M. Cohen, R. Huynh, M. Landuré, R. Lehner, J.-C. Margiotta, M. Parmentier, C. Rebattu, C. Schmerber, M. Sénéquier, J. et W. Williams, J. Woodbury.

Ouvriers: A. Ben Youssef Mosbah, A. Hadjaad, H. Hadj Mahmoud, M. Hamadi, A. Ilajsaad, B. Ilamouri, M. Kamel Hadj, A. Mosbah.

Assistance technique : E. Durand, Services techniques municipaux de Fréjus.

Cette étude a bénéficié du concours technique de : A. Dupeyroux, pour les tirages photographiques noir et blanc; P. Foliot,
CCJ, C.NRS, Aix-en-Provence, fig. 18, 34, 36 et 38; A.-D. Gallizia, photographie fig. 13; J.-M. Gassend et J. Bigot, IRAA, C.NRS Aix-en-Provence, fig. 16 et $28 ;$ M. Heller, Service de l'Inventaire des Monuments historiques, Aix-en-Provence, photographies aériennes, fig. 2 ; C. Orban, Services techniques municipaux de Fréjus, mise à l'échelle des plans des fouilles de 1829,1986 et 1988 ; S. Osenda, Services techniques municipaux de Fréjus, relevés et restitutions topographiques du chantier de la Porte d'Orée; K. Vaiden-Gicquel, dessins des céramiques; $\mathrm{J}$. Woodbury, mise au net de plan et étude partielle des céramiques fines.

Nous remercions : G. Bèrard, J. Bigot, M. Bonifay, H. Broise, J.-P. Brun, A. Carrier, G. Congès, A. Dupeyroux, A.-D. Galli7.ia, J.-M. Gassend, M.-F. Giaccobi-Lequément, P.-A. Février, M. Leguilloux, B. Liou, M. Pasqualini, J.-P. Pelletier, J. et Y. Rigoir, A. Roth Congès, A. Tchernia, L. Vallauri et plus particulièrement $R$. Boiron. 


\section{BIBLIOGRAPHIE}

Adam J.-P.

1984 : La construction romaine. Matériaux el techniques, Paris, Picard, 367 p., 746 fig.

Amouric H., Landuré C.

1985 : Archives et archéologie : l'exemple de l'artisanat céramique à Fréjus, Provence Historique, XXXV, 141, p. 299-308.

Aubenas J.-A.

1881 : Histoire de Fréjus, Marseille, 1974 réédition Laffitte, 800 p., 2 pl.

\section{Aupert P.}

1974 : Le nymphée de Tipasa, Collection de l'École Française de Rome, 16, 167 p., fig., plans et tabl.

\section{Bérato J., Bérato N.}

1982 : Le site gallo-romain de la Meynade-Le Clafard à SixFours (Var), Annales de la Société des Sciences Naturelles el d'Archéologie de Toulon et du Var, 34, p. 50-77.

\section{Bérato $\mathrm{J}$.}

1984 : Évolution de la céramique modelée de la fin de la protohistoire à la période gallo-romaine, sur les sites de Taradeau (Var), Annales de la Société des Sciences Naturelles el d'Archéologie de Toulon et du Var (ASSNATV), 36, p. 217-224.

Bérato J., Borréani M., Brun J.-P., Pasqualini M., Pollino A. 1986a : L'épave des Roches d'Aurelles, L'exploitation de la mer, la mer moyen d'échange el de communicalion, Actes des VI'Rencontres Internationales d'Archéologie et d'Histoire d'Antibes, Octobre 1985, Valbonne, p. 191-216.

Bérato J., Borréani M., Lecacheur P., Pasqualini M., Théveny J.-M., Rigoir J., Rigoir Y.

1986b : Fouilles récentes à Toulon (Var) (Quartier Besagne, 1985-1986), Documents d'Archéologie Méridionale, 9, p. 135166.

\section{Béraud I., Gébara Ch.}

1986 : Les lits funéraires de la nécropole gallo-romaine de Saint-Lambert (Fréjus), Revue Archéologique de Narbonnaise (RAN), 19, p. 183-210.

Blackman D.J.

1973 : Evidence of Sea Level Change in Ancient Harbours and Coastal Installations, Marine Archaeology, Colston Papers, 23, p. 115-137.

1982 : Ancient Harbours in the Mediterranean, Part 2, Journal of the International Nautical Archaeology and Under-water Exploration, II, p. 79-104 et p. 185-211.

Bonifay $\mathrm{M}$.

1983 : Éléments d'évolution des céramiques tardives à Marseille d'après les fouilles de la Bourse (1980-1981), RAN, 16 . p. 285-346.

Borréani M., Brun J.-P., Lecacheur P., Pasqualini M. 1987 : Travaux du Centre Archéologique de Toulon et du Var, ASSNATV, p. 3-22.
Brun J.-P., Congès G., Prothro K.

1986 : Un foyer d'époque mérovingienne dans la villa de Pardigon 2 (Var-la Croix-Valmer), RAN, 19, p. 307-317.

\section{Carandini A.}

1981 : Allante delle forme ceramiche, I, Ceramica fine nel bacino medilerraneo (medio e lardo impero), suppl. à l'Enciclopedia del Arte Antica, Rome, 270 p., $165 \mathrm{pl}$.

CATHMA (Céramiques de l'Antiquité Tardive et du llaut Moyen-Age)

1984 : La céramique du Haut Moyen-Age en France méridionale : éléments comparatifs et essai d'interprétation, La ceramica medievale nel Medilerraneo Occidentale, Actes du Colloque International de Sienne-Faenza, Florence, p. 57-62.

Sous presse : Importations de céramiques communes méditerranéennes dans le Midi de la Gaule ( $\mathrm{v}^{\mathrm{c}}-\mathrm{vIII}^{\mathrm{c}} \mathrm{s}$.), Colloque International de la Céramique Médiévale, Lisbonne.

Cavailles-Llopis M.-T.

1986 : Céramiques de l'Antiquité tardive à Marseille (corne du port antique, sondages D II 11 et D II 15), Documents $d$ 'Archéologie Méridionale, 9, p. 167-195.

Démians d'Archimbaud G.

1980 : Les fouilles de Rougiers, contribution à l'archéologie de l'habital rural, Paris, Éd. du C.NRS, 724 p., 520 fig.

Desbat A., Laroche C., Mérigoux E.

1979 : Note préliminaire sur la céramique commune de la rue des Farges à Lyon, Figlina, 4, p. 1-17.

Dutour O., Bérato J., Williams J.

1991 : Sépultures du site antique de la Porte d'Orée, Fréjus. Étude anthropologique et pathologique, L'Anthropologie, 95, 2. p. $653-662$.

\section{Dyson S.L.}

1976 : Cosa : the Utilitarian Pottery, American Academy in Rome, Rome, 173 p., 68 fig., 2 pl.

Faure-Boucharlat E., Colardelle M., Fixot M., Pelletier J.-P. 1978 : Eléments comparatifs de la production céramique du $\mathrm{XI}^{\mathrm{e}} \mathrm{s}$. dans le bassin rhodanien, La ceramica medievale nel Mediterraneo Occidentale, Actes du Colloque International de Valbonne, Paris, p. 429-440.

Février P.-A.

1956a : Fouilles à la citadelle méridionale de Forum Julii (Fréjus, Var) en 1955, Gallia, 14, p. 35-54.

1956b : Les appareils des murs romains de Fréjus (Introduction à une étude des constructions de la Provence orientale), Revue d'Études Ligures, XXII, 2-4, p. 153-184.

Fixot M.

1980 : Découvertes récentes de silos médiévaux en Provence, Provence Historique, XXIX, 118, p. 387-404. 
Fixot M., Pelletier J.-P.

1983 : Une forme originale de fortification médiévale provençale : le Castellas de Cucuron (Vaucluse), Archéologie Médiévale, XIII, p. 89-115.

Fixot M., Guyon J., Pelletier J.-P., Rivet L.

1986 : Des abords du forum au palais archiépiscopal, ètude du centre monumental d'Aix-en-Provence, Bulletin Monumental, 144, III, p. 196-290.

Flemming N.C., Czartoryska N.M.G., Hunter P.M.

1973 : Eustatic and Tectonic Components of Relative Sea Level Change, Marine Archaeology, Colston Papers, 23, p. 62.

\section{Fulford M.G., Peacock D.P.S.}

1984 : The avenue du Président Habib Bourguiba, Salambo : The pottery and other ceramic objects from the sile, Excavations at Carthage: The British Mission, volume 1, 2, British Academy. University of Sheffield, 284 p., $96 \mathrm{fig}$., $6 \mathrm{pl}$.

Glaser F.

1983: Antike Brunnenbauten (KPIIYAI) in Griechenland, Österreichischen Akademie der Wissenschaften.

\section{Goudineau Chr.}

1981 : Une fouille de sauvetage aux Aiguières, Les Dossiers: Histoire el Archéologie, 57, p. 52-54.

1982 : Une fouille récente à la périphérie de Forum Julii : le chantier des Aiguières, Comptes Rendus de l'Academie des Inscriptions et Belles-Lettres, avril-juin, p. 279-292.

Hayes J.W.

1972 : Late Roman Poltery, Londres, 477 p., 93 fig., 23 pl., 40 cartes.

\section{Heraclea Caccabaria}

1988: Autour d'Heraclea Caccabaria. Archéologie de la côte des Maures, catalogue de l'exposition, Centre de Documentation Archéologique du Var, Toulon, 64 p., 196 fig.

\section{Jashemski W.F.}

1975: The Gardens of Pompeii, Chronache Pompeiane, I, p. 48-81.

Jonot A., Villes A.

1976 : Une structure de I'habitat protohistorique en Champagne crayeuse : le silo, Bulletin de la Socièlé Archéologique Champenoise, 4, p. 25-38.

1981 : Fosses d'ensilage dans l'habitat protohistorique en Champagne crayeuse, Mémoires de la Socièté d'Agriculture, Commerce, Sciences et Arts du Département de la Marne, XCVI, p. 21-25.

Keay S.J.

1984 : Late Roman Amphorae in the Western Medilerranean. A Typology and Economic Study: the Catalan Evidence, B.A.R., International Series, 196 (i) (ii), 738 p., 216 fig.

Laubenhcimer F.

1991 : Les vides sanitaires et les amphores de la Porte d'Orée à Fréjus (Var), Gallia, 48, p. 229-265.

Lavagne $\mathrm{H}$.

1988 : Operosa Antra. Recherches sur la grotte à Rome de Sylla à IIadrien, BEFAR, 272, X-752 p.

Le Gall J.

1952 : Le Tibre fleuve de Rome dans l'Antiquité, Paris.

Leguilloux M.

1988: Les amphores romaines, guide typologique, II, les amphores à vin tardives, IV ${ }^{\mathrm{e}}-\mathrm{v} \mathrm{II}^{\mathrm{r}}$ s. après J.-C., ASSNATV, 40, p. 31-40.

\section{Nécropoles de Fréjus}

1985 : Les nécropoles gallo-romaines de Fréjus. Trois années d'action du service archéologique municipal, catalogue de l'exposition, Saint-Raphaël, Comité d'action et d'animation culturelles.

Neuerburg $\mathrm{N}$.

1965 : L'architeltura delle fontane e dei ninfei nell'Italia antica, Naples.

Nin N., De Luca B.

1987 : La voie aurélienne et ses abords, Iocuments d'Archéologie Aixoise, 3, Aix-en-Provence, 63 p., 82 fig.

\section{Pasqualini $\mathbf{M}$}

1988: Céramiques dans le bassin de l'Argens et la région de Fréjus (Var) entre le $\mathrm{I}^{\mathrm{er}}$ et le $\mathrm{III}^{\mathrm{C}} \mathrm{s}$. de notre ère. Les productions régionales, Socièté Française pour l'Étude de la Céramique Antique en Gaule, Actes du congrès d'Orange 12-15 mai 1988, p. $155-168$.

\section{Peacock D.P.S., Williams D.F.}

1986 : Amphorae and the Roman Economy, Londres, 239 p., 139 fig.

Pelletier J.-P., Vallauri L.

à paraître : La céramique commune grise, in: G. Démians d'Archimbaud (dir.), L'oppidum de Saint-Blaise, Bouches-duRhône: la réoccupation aux $V^{e-V I I^{e}} s$. d'après les fouilles récentes, DAF. Paris, Éditions de la Maison des Sciences de l'Homme.

Ports et villes engloutis

1981 : Ports et villes engloutis. Variations du niveau de la mer et séismes dans l'Antiquité, Les Dossiers : IIistoire et Arrhéologie, 50, 87 p.

Rigoir J.

1960 : La céramique paléochrétienne sigillée grise, Provence IIistorique, X, 42, p. 1-93.

Rigoir J., Rigoir Y.

1985 : Dérivées des sigillées paléochrétiennes provençales. Catalogue des formes, Paris.

Rivet L.

1980 : Fouilles du Clos de la Tour à Fréjus (Var): les rues, Thèse de $3^{\mathrm{e}}$ cycle, Aix-en-Provence, dactyl., 915 p., $212 \mathrm{pl}$., 89 fig., 25 photos.

1982 : La céramique culinaire micacée de la région de Fréjus (Var), $R A N, 15$, p. 243-262.

à paraître: La céramique commune du site de VilleneuveAiguières à Fréjus.

Santamaria C.

1985: L’épave Chrétienne II, Agay, commune de SaintRaphaël, Provence Ilistorique, $\mathrm{XXXV}, 141$, p. 267-261, $6 \mathrm{pl}$.

Testaguzza $\mathrm{O}$.

1970: Portus, illustrazione dei porti di Claudio e Traiano, Rome.

Vallauri L.

a paraitre : Céramiques communes importées et d'origine indéterminée, in : G. Démians d'Archimbaud (dir.), L'oppidum de Saint-Blaise, Bouches-du-Rhône: la réoccupation aux "v-vor's. d'après les fouilles récentes, DAF, Paris, Éditions de la Maison des Sciences de l'Homme. 